\title{
The Morse index of a triply periodic minimal surface
}

\author{
Norio Ejiri * Toshihiro Shoda ${ }^{\dagger}$
}

\begin{abstract}
In the previous work, the first author established an algorithm to compute the Morse index and the nullity of an $n$-periodic minimal surface in $\mathbb{R}^{n}$. In fact, the Morse index can be translated into the number of negative eigenvalues of a real symmetric matrix and the nullity can be translated into the number of zero-eigenvalue of a Hermitian matrix. The two key matrices consist of periods of the abelian differentials of the second kind on a minimal surface, and the signature of the Hermitian matrix gives a new invariant of a minimal surface. On the other hand, $\mathrm{H}$ family, rPD family, tP family, tD family, and tCLP family of triply periodic minimal surfaces in $\mathbb{R}^{3}$ have been studied in physics, chemistry, and crystallography. In this paper, we first determine the two key matrices for the five families explicitly. As its applications, by numerical arguments, we compute the Morse indices, nullities, and signatures for the five families.
\end{abstract}

\section{Introduction}

The Morse index (resp. the nullity) of a compact oriented minimal submanifold in an oriented Riemannian manifold is defined as the sum of the dimensions of the eigenspaces corresponding to negative eigenvalues (resp. zero-eigenspace) of the Jacobi operator of the area. The purpose of this work is to compute the Morse index and the nullity of a compact oriented minimal surface in a flat three-torus. Moreover, we consider a signature of a minimal surface defined in [3, and compute the signature of a compact oriented minimal surface in a flat three-torus. Now we refer to backgrounds.

In 1968, Simons [13] gave the second variational formula of the area and compute the Morse index and the nullity of a totally geodesic subsphere in the sphere. By his technique, we can see that the Morse index (resp. the nullity) of a totally geodesic subtorus in a flat three-torus is zero (resp. one). Next impressive developments were obtained by Montiel-Ros [8] and Ross [10]. Montiel-Ros

\footnotetext{
*Partly supported by JSPS Grant-in-Aid for Scientific Research (C) $15 \mathrm{~K} 04859$.

$\dagger^{\dagger}$ Partly supported by JSPS Grant-in-Aid for Scientific Research (C) 16K05134.

2000 Mathematics Subject Classification. Primary 53A10; Secondary 49Q05, 53C42.

Key words and phrases. minimal surfaces, flat tori, Morse index.
} 
considered the Dirichlet eigenvalue problem and the Neumann eigenvalue problem of the Laplacian to compute the Morse index and the nullity of a minimal surface. Their study includes the result that tCLP family consists of minimal surfaces with Morse index three and nullity three. Ross proved that Schwarz P surface, D surface, and Schoen's Gyroid are volume preserving stable, respectively. Applying his arguments, we find that each of the three minimal surfaces has Morse index one and nullity three. But the Morse index and the nullity have not been computed for other examples in the past two decades.

Recently, the first author [2, 3] established a Moduli theory of compact oriented minimal surfaces in flat tori via the Morse index and the nullity. Also, he gave a procedure to compute the Morse index of a minimal surface with only trivial Jacobi fields. The procedure is to reduce computing the Morse index and the nullity of such minimal surfaces to fundamental arguments of eigenvalues in linear algebra. The main difficulty is to determine a canonical homology basis and the period matrix for each minimal surface explicitly. Our technique developed in this paper is to overcome this by using the method which is faithful to the basics of compact Riemann surfaces, and shows that the procedure turns out to be practical. Recall that a normal vector field vanishing the Jacobi operator of the area is called a Jacobi field and the dimension of the space of Jacobi fields is equal to the nullity of a minimal surface. It is well-known that normal components of the Killing vector fields generated by translation on the torus give rise to Jacobi fields. So if we consider a non-totally geodesic compact oriented minimal surface in a flat $n$-torus $\mathbb{R}^{n} / \Lambda$, then it has nullity at least $n\left(=\operatorname{dim} \mathbb{R}^{n} / \Lambda\right)$. The Killing vector fields generated by translation on the torus is called trivial Jacobi fields, and a minimal surface has only trivial Jacobi fields if and only if its nullity is equal to $n$. Moreover, he introduced a new invariant for the Moduli theory of compact oriented minimal surfaces in flat tori which is called a signature of a minimal surface (see Section Two for the detail). The present work suggests that it might be easier to compute the signature than the Morse index.

On the other hand, triply periodic minimal surfaces in $\mathbb{R}^{3}$ have been studied in physics, chemistry, and crystallography. Schröder-Turk, Fogden, and Hyde 14] studied one-parameter families of triply periodic minimal surfaces in $\mathbb{R}^{3}$. These one-parameter families which are called $\mathrm{H}$ family, rPD family, tP family, tD family, and tCLP family, contain many classical examples (Schwarz P surface, D surface, Schwarz H surface, and Schwarz CLP surface). Note that a triply periodic minimal surface properly immersed in $\mathbb{R}^{3}$ corresponds to a minimal immersion of a compact oriented surface into a flat three-torus. Hence the above one-parameter families are related to our works. Also, the above five families consist of minimal surfaces of genus three, and the following Ros' result clarifies an importance of such families. In fact, in this case, the minimum value of Morse index must be one (see Remark 4.1) and Ros 9] proved that genus of a compact oriented minimal surface in a flat three-torus with Morse index one must be three. In the present paper, we compute the Morse index, the nullity, and the signature of each of the above one-parameter families.

To state our main results, we review some fundamental arguments in the 
theory of minimal surfaces. Let $f: M \rightarrow \mathbb{R}^{n} / \Lambda$ be a minimal immersion of a compact oriented surface $M$ into a flat $n$-torus $\mathbb{R}^{n} / \Lambda$. With the induced conformal structure, $M$ is a compact Riemann surface and $f$ is called a conformal minimal immersion. Our object is then to study conformal minimal immersions of compact Riemann surfaces in flat $n$-tori. For a conformal minimal immersion, the following theorem is a basic tool.

Theorem 1.1 (Weierstrass representation formula).

Let $f: M \rightarrow \mathbb{R}^{n} / \Lambda$ be a conformal minimal immersion. Then, up to translations, $f$ can be represented by the following path-integrals:

$$
f(p)=\operatorname{Re} \int_{p_{0}}^{p}\left(\omega_{1}, \ldots, \omega_{n}\right)^{t} \bmod \Lambda,
$$

where $p_{0}$ is a fixed point on $M$ and the $\omega_{i}$ 's are holomorphic differentials on $M$ satisfying the following three conditions.

$$
\begin{aligned}
& \omega_{1}^{2}+\cdots+\omega_{n}^{2}=0 \\
& \omega_{1}, \ldots, \omega_{n} \text { have no common zeros, } \\
& \left\{\operatorname{Re} \int_{C}\left(\omega_{1}, \ldots, \omega_{n}\right)^{t} \mid C \in H_{1}(M, \mathbb{Z})\right\} \text { is a sublattice of } \Lambda .
\end{aligned}
$$

Conversely, the real part of path-integrals of holomorphic differentials satisfying the above three conditions defines a conformal minimal immersion of $M$ into a flat $n$-torus.

Let index $_{A}$ (resp. nullity $_{A}$ ) denote the Morse index (resp. the nullity) of a minimal surface, and $(p, q)$ the signature of a minimal surface. Now we refer to our main results as follows.

Main Theorem 1 (H family).

For $a \in(0,1)$, let $M$ be the hyperelliptic Riemann surface of genus three defined by $w^{2}=z\left(z^{3}-a^{3}\right)\left(z^{3}-1 / a^{3}\right)$ and $f$ the conformal minimal immersion given by

$$
f(p)=\operatorname{Re} \int_{p_{0}}^{p} i\left(1-z^{2}, i\left(1+z^{2}\right), 2 z\right)^{t} \frac{d z}{w} .
$$

Then there exist $0<a_{1}<a_{2}<1$ satisfying the following properties:

(i) index $_{A}=2$, nullity $A=3$, and $(p, q)=(5,4)$ for $a \in\left(0, a_{1}\right)$,

(ii) index $_{A}=1$, nullity $_{A}=4$, and $(p, q)=(4,4)$ for $a=a_{1}$,

(iii) index $_{A}=1$, nullity $_{A}=3$, and $(p, q)=(4,5)$ for $a \in\left(a_{1}, a_{2}\right)$,

(iv) index $_{A}=1$, nullity $_{A}=5$, and $(p, q)=(4,3)$ for $a=a_{2}$,

(v) index $_{A}=3$, nullity $_{A}=3$, and $(p, q)=(6,3)$ for $a \in\left(a_{2}, 1\right)$,

where $a_{1} \approx 0.49701, a_{2} \approx 0.71479$. We obtain the similar results for $a>1$.

Main Theorem 2 (rPD family, Karcher TT surface).

For $a \in(0,1]$, let $M$ be the hyperelliptic Riemann surface of genus three defined by $w^{2}=z\left(z^{3}-a^{3}\right)\left(z^{3}+1 / a^{3}\right)$ and $f$ the conformal minimal immersion 
given by

$$
f(p)=\operatorname{Re} \int_{p_{0}}^{p} i\left(1-z^{2}, i\left(1+z^{2}\right), 2 z\right)^{t} \frac{d z}{w} .
$$

The case $a=1 / \sqrt{2}$ corresponds to Schwarz $\mathrm{P}$ surface.

Then there exist $0<a_{1}<1$ satisfying the following properties:

(i) index $_{A}=2$, nullity $_{A}=3$, and $(p, q)=(5,4)$ for $a \in\left(0, a_{1}\right)$,

(ii) index $_{A}=1$, nullity $_{A}=4$, and $(p, q)=(4,4)$ for $a=a_{1}$,

(iii) index $_{A}=1$, nullity $_{A}=3$, and $(p, q)=(4,5)$ for $a \in\left(a_{1}, 1\right]$,

where $a_{1} \approx 0.494722$. We obtain the similar results for $a \geq 1$.

\section{Remark 1.2.}

$a_{1}$ 's in Main Theorem 1 and Main Theorem 2 are considered from other point of view in 14 .

Main Theorem 3 (tP family, tD family).

For $a \in(2, \infty)$, let $M$ be the hyperelliptic Riemann surface of genus three defined by $w^{2}=z^{8}+a z^{4}+1$ and $f$ the conformal minimal immersion given by

$$
f(p)=\operatorname{Re} \int_{p_{0}}^{p}\left(1-z^{2}, i\left(1+z^{2}\right), 2 z\right)^{t} \frac{d z}{w} .
$$

The case $a=14$ corresponds to Schwarz $\mathrm{P}$ surface.

Then there exist $2<a_{1}<14<a_{2}<\infty$ satisfying the following properties:

(i) index $_{A}=2$, nullity $_{A}=3$, and $(p, q)=(5,4)$ for $a \in\left(2, a_{1}\right)$,

(ii) index $_{A}=1$, nullity $_{A}=3$, and $(p, q)=(4,5)$ for $a \in\left(a_{1}, a_{2}\right)$,

(iii) index $_{A}=2$, nullity $A=3$, and $(p, q)=(5,4)$ for $a \in\left(a_{2}, \infty\right)$,

(iv) index $_{A}=1$, nullity $_{A}=4$, and $(p, q)=(4,4)$ for $a=a_{1}, a_{2}$,

where $a_{1} \approx 7.40284, a_{2} \approx 28.7783$.

$t D$ family is defined as a family of conjugate surfaces of minimal surfaces which belong to tP family. Thus we obtain the same result for tD family as the above.

Main Theorem 4 (tCLP family).

For $a \in(-2,2)$, let $M$ be the hyperelliptic Riemann surface of genus three defined by $w^{2}=z^{8}+a z^{4}+1$ and $f$ the conformal minimal immersion given by

$$
f(p)=\operatorname{Re} \int_{p_{0}}^{p}\left(1-z^{2}, i\left(1+z^{2}\right), 2 z\right)^{t} \frac{d z}{w} .
$$

The case $a=0$ corresponds to Schwarz CLP surface.

Then, for an arbitrary $a \in(-2,2)$, index $x_{A}=3$, nullity $_{A}=3$, and $(p, q)=$ $(6,3)$.

Our main theorems imply that every compact oriented minimal surface which belongs to the above five families has Morse index at most three, and we obtain the same results for other families as well [5]. So we propose the following "one-two-three conjecture": 


\section{Conjecture.}

If a compact oriented minimal surface in a flat three-torus has genus three, then we have $1 \leq$ index $_{A} \leq 3$.

The outline of the paper as follows: The second section contains a short story of the procedure to compute the Morse index and the nullity. Section Three gives explicit descriptions of the key matrices to compute the Morse index and the nullity for each one-parameter family. Section Four contains details of numerical arguments of our main results by Mathematica, and finally in Section Five there is a collection of calculations to determine a canonical homology basis and the period matrix for each one-parameter family of minimal surfaces as appendix.

The authors would like to thank Wayne Rossman and Shoichi Fujimori for useful conversations about Mathematica.

\section{An algorithm to compute the Morse index and the nullity}

In this section, we refer to a story to compute the Morse index and the nullity of a minimal surface in a flat torus. The details of the contents are given in [2, 3] (see also [4 for its outline).

Let $R$ be a compact oriented surface of genus $\gamma$ and $\phi: R \rightarrow \mathbb{R}^{n} / \Lambda$ a smooth map. Schoen and Yau [12] defined an energy $E_{\phi}$ on the Teichmüller space $\mathcal{T}_{\gamma}$ with base surface $R$. Recall that a point in $\mathcal{T}_{\gamma}$ is an equivalent class of pairs $[(M, h)]$ of a compact Riemann surface $M$ of genus $\gamma$ and an orientation preserving diffeomorphism $h: R \rightarrow M$. The following result suggests that the energy $E_{\phi}$ is one of important objects in Differential Geometry.

Theorem 2.1 ([11, 12]). A critical point $p=(M, h) \in \mathcal{T}_{\gamma}$ of $E_{\phi}$ is corresponding to a conformal (branched) minimal immersion $f: M \rightarrow \mathbb{R}^{n} / \Lambda$.

Since $\mathcal{T}_{\gamma}$ is diffeomorphic to $\mathbb{R}^{6 \gamma-6}$, we can define the Morse index and the nullity of $E_{\phi}$ at a critical point by the Hessian. Let index $E_{E}$ denote the Morse index of $E_{\phi}$ at a critical point and nullity $E_{E}$ the nullity of $E_{\phi}$ at a critical point. We can translate $i n d e x_{a}$ (resp. nullitya) into index $_{E}$ (resp. nullity $y_{E}$ ) as follows.

Theorem 2.2 (Theorem 3.4 in [2]). Suppose that $f: M \rightarrow \mathbb{R}^{n} / \Lambda$ is a conformal minimal immersion and $p=[(M, h)] \in \mathcal{T}_{\gamma}$ is the corresponding critical point of $E_{\phi}$. Then

$$
\text { index }_{a}=\text { index }_{E}, \quad \text { nullity }_{a}=\text { nullity }_{E}+n .
$$

As an immediate consequence, we have

Corollary 2.3 (Corollary 3.20 in [2]). Suppose that $f: M \rightarrow \mathbb{R}^{n} / \Lambda$ is a conformal minimal immersion and $p=[(M, h)] \in \mathcal{T}_{\gamma}$ is the corresponding critical point of $E_{\phi}$. If $f$ has only trivial Jacobi fields, then $E_{\phi}$ is non-degenerate at $p$. 
Before we refer to an algorithm to compute index $_{E}$ and nullity $_{E}$, we review some arguments.

Let $\left\{A_{j}, B_{j}\right\}_{j=1}^{\gamma}$ be a canonical homology basis and $\left\{\varphi_{1}, \cdots, \varphi_{\gamma}\right\}$ a basis of the space of holomorphic differentials on a compact Riemann surface $M$. We set $\Phi=\left(\varphi_{1}, \cdots, \varphi_{\gamma}\right)^{t}$. Then there exists a unique basis $\left\{\varphi_{j}\right\}_{j=1}^{\gamma}$ with the following property:

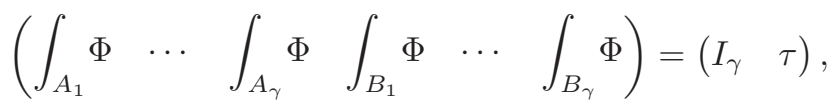

where $I_{\gamma}$ is the identity matrix of degree $\gamma$ and $\tau$ is a complex symmetric matrix of degree $\gamma$ with $\operatorname{Im} \tau>0$. This $\tau$ is called a Riemann matrix of $M$. Let $L_{m, n}$ be a set of real $(m, n)$ matrices and $K_{m, n}$ a set of complex $(m, n)$ matrices. Suppose that $f(p)=\operatorname{Re}\left(\begin{array}{lll}\int_{p_{0}}^{p} \omega_{1} & \cdots & \int_{p_{0}}^{p} \omega_{n}\end{array}\right)^{t}$ is a conformal minimal immersion of a Riemann surface $M$ as in Theorem 1.1. Then we call

$$
\operatorname{Re}\left(\begin{array}{llllll}
\int_{A_{1}} \omega_{1} & \cdots & \int_{A_{\gamma}} \omega_{1} & \int_{B_{1}} \omega_{1} & \cdots & \int_{B_{\gamma}} \omega_{1} \\
\int_{A_{1}} \omega_{n} & \cdots & \int_{A_{\gamma}} \omega_{n} & \int_{B_{1}} \omega_{n} & \cdots & \int_{B_{\gamma}} \omega_{n}
\end{array}\right) \in L_{n, 2 \gamma}
$$

a real period matrix and

$$
\left(\begin{array}{llllll}
\int_{A_{1}} \omega_{1} & \cdots & \int_{A_{\gamma}} \omega_{1} & \int_{B_{1}} \omega_{1} & \cdots & \int_{B_{\gamma}} \omega_{1} \\
\int_{A_{1}} \omega_{n} & \cdots & \int_{A_{\gamma}} \omega_{n} & \int_{B_{1}} \omega_{n} & \cdots & \int_{B_{\gamma}} \omega_{n}
\end{array}\right) \in K_{n, 2 \gamma}
$$

a complex period matrix. Let $L \in L_{n, 2 \gamma}$ be a real period matrix of $f$. By using a decomposition $L=\left(L_{1}, L_{2}\right)\left(L_{j} \in L_{n, \gamma}\right)$,

$$
\left(\begin{array}{lll}
\omega_{1} & \cdots & \omega_{n}
\end{array}\right)^{t}=\frac{1}{2}\left(L_{1}+i\left[L_{1} \operatorname{Re} \tau-L_{2}\right](\operatorname{Im} \tau)^{-1}\right) \Phi
$$

holds (see $\S 7$ in [2], see also p.161 in [4). Note that we have to assume (1.1) for the above. By setting

$$
K(\tau, L)=\frac{1}{2}\left(L_{1}+i\left[L_{1} \operatorname{Re} \tau-L_{2}\right](\operatorname{Im} \tau)^{-1}\right)
$$

and (2.1), the complex period matrix can be written as

$$
(K(\tau, L) \quad K(\tau, L) \tau) \in K_{n, \gamma} \times K_{n, \gamma}=K_{n, 2 \gamma} .
$$

Using (2.2), we can construct a complex isotropic cone in $K_{n, \gamma} \times K_{n, \gamma}=K_{n, 2 \gamma}$. Moreover, if a minimal surface has only trivial Jacobi fields, then the complex 
isotropic cone defined by (2.2) must be complex Lagrangian (see Theorem 8.1 in [3, see also p.166 in [4).

To compute index $_{E}$ and nullity $y_{E}$, we consider a basis of a tangent space of the complex isotropic cone by (2.2) derived from deformations of a complex structure of $M$ and an action of $S O(n, \mathbb{C}) \times(\mathbb{C} \backslash\{0\})$ (see p.169 in [4]). The basis are given by periods of the abelian differentials of the second kind. Recall that the abelian differentials of the second kind are meromorphic differentials with zero residues on a Riemann surface. Let $\left\{T_{j}\right\}_{j=1}^{n \gamma}$ be the basis of the tangent space of the complex isotropic cone by (2.2) in $K_{n, \gamma} \times K_{n, \gamma}=K_{n, 2 \gamma}$, and we shall determine $\left\{T_{j}\right\}_{j=1}^{n \gamma}$ for each one-parameter family explicitly in the next section. Define

$$
\eta\left(\left(Z_{1}, Z_{2}\right),\left(Z_{1}^{\prime}, Z_{2}^{\prime}\right)\right):=-i \operatorname{tr}\left(\left(Z_{2}^{t}\right) \overline{Z_{1}^{\prime}}-\left(Z_{1}^{t}\right) \overline{Z_{2}^{\prime}}\right)
$$

for $\left(Z_{1}, Z_{2}\right),\left(Z_{1}^{\prime}, Z_{2}^{\prime}\right) \in K_{n, \gamma} \times K_{n, \gamma}$ and $W:=\left(\eta\left(T_{i}, T_{j}\right)\right)$. Note that $\operatorname{Re} \eta$ is a pseudo Kähler metric given in [1] (see $\S 9$ in [3], see also p.167 in [4). $W$ is the Hermitian matrix of degree $n \gamma$ and it is one of the key matrices as we referred to in the abstract. We call a pair of the number of positive eigenvalues and that of negative eigenvalues of $W$ counted with multiplicities the signature of a minimal surface, and let $(p, q)$ denote the signature of a minimal surface. For a decomposition $T_{j}=\left(C_{j}, D_{j}\right)\left(C_{j}, D_{j} \in K_{n, \gamma}\right)$, we set

$$
\begin{aligned}
K_{j}^{\prime} & :=\operatorname{Re} C_{j}+i\left\{\operatorname{Re} C_{j} \operatorname{Re} \tau-\operatorname{Re} D_{j}\right\}(\operatorname{Im} \tau)^{-1}, \\
K_{j}^{\prime \prime} & :=\operatorname{Re}\left(i C_{j}\right)+i\left\{\operatorname{Re}\left(i C_{j}\right) \operatorname{Re} \tau-\operatorname{Re}\left(i D_{j}\right)\right\}(\operatorname{Im} \tau)^{-1} .
\end{aligned}
$$

Define

$$
\begin{aligned}
U_{k} & := \begin{cases}T_{k} & (1 \leq k \leq n \gamma) \\
i T_{k-n \gamma} & (n \gamma+1 \leq k \leq 2 n \gamma)\end{cases} \\
V_{k} & := \begin{cases}\left(K_{k}^{\prime}, K_{k}^{\prime} \tau\right) & (1 \leq k \leq n \gamma) \\
\left(K_{k-n \gamma}^{\prime \prime}, K_{k-n \gamma}^{\prime \prime} \tau\right) & (n \gamma+1 \leq k \leq 2 n \gamma)\end{cases}
\end{aligned}
$$

and $W_{1}:=\left((\operatorname{Re} \eta)\left(U_{i}, U_{j}\right)\right), W_{2}:=\left((\operatorname{Re} \eta)\left(V_{i}, V_{j}\right)\right)$. Each $W_{j}$ is a real symmetric matrix of degree $2 n \gamma$, and $W_{2}-W_{1}$ is another key matrix.

Now we refer to an algorithm to compute the Morse index and the nullity of a minimal surface, that is, index $x_{E}$ and nullity $_{E}$ for the one-parameter families in the introduction. It is well-known that, for the case $\gamma=n=3$, the Riemann surface $M$ is hyperelliptic (see Corollary 3.2 in [7). Since we consider a family of minimal surfaces parametrized by $a$ which belongs to a suitable interval $I \subset \mathbb{R}$, $W$ and $W_{2}-W_{1}$ are also parametrized by $a$. nullity $y_{E}$ is equal to the number of zero eigenvalues of $W$ counted with multiplicities (see Theorem 7.10 and $\S 14$ in [2], Theorem 9.5 in [3]). Thus the set $\{a \in I \mid \operatorname{det} W=0\}$ coincides with the set $\left\{a \in I \mid\right.$ nullity $\left._{E} \neq 0\right\}$ and it divides $I$ into some intervals. Note that $(p, q)$ and $i n d e x_{E}$ are constant on each divided interval. If the dimension of the zero eigenspace of $W_{2}-W_{1}$ is equal to $(2 n-4) \gamma+2=8$, then $i n d e x_{E}-1$ is equal to the number of negative eigenvalues of $W_{2}-W_{1}$ counted with multiplicities (see 
Theorem 7.10, $\S 14$ in [2], Theorem 9.6 in [3], see also p.170 in [4). Every family of minimal surfaces which we treat in this paper satisfies this assumption.

We will use the notation $T_{j}, W, W_{j}$ below.

\section{$3 \quad$ Key matrices}

\subsection{H family}

We start from the following Lemmas.

\section{Lemma 3.1.}

$$
\begin{array}{r}
d\left(z^{\alpha} w^{\beta}\right)=\frac{1}{2} z^{\alpha-1} w^{\beta-2}\left\{(2 \alpha+7 \beta) w^{2}+3 \beta\left(a^{3}+1 / a^{3}\right) z^{4}-6 \beta z\right\} d z \\
=\frac{1}{2} z^{\alpha-1} w^{\beta-2}\left\{(2 \alpha+7 \beta) z^{7}-(2 \alpha+4 \beta)\left(a^{3}+1 / a^{3}\right) z^{4}\right. \\
+(2 \alpha+\beta) z\} d z .
\end{array}
$$

Proof. Straightforward calculation.

\section{Lemma 3.2.}

For an arbitrary 1-cycle $\gamma$, we have the following formulae.

$$
\begin{aligned}
\int_{\gamma} \frac{z}{w^{3}} d z & =\frac{5}{6} \int_{\gamma} \frac{d z}{w}+\frac{1}{2}\left(a^{3}+\frac{1}{a^{3}}\right) \int_{\gamma} \frac{z^{4}}{w^{3}} d z \\
\int_{\gamma} \frac{z^{2}}{w^{3}} d z & =\frac{1}{2} \int_{\gamma} \frac{z}{w} d z+\frac{1}{2}\left(a^{3}+\frac{1}{a^{3}}\right) \int_{\gamma} \frac{z^{5}}{w^{3}} d z \\
\int_{\gamma} \frac{z^{3}}{w^{3}} d z & =\frac{1}{6} \int_{\gamma} \frac{z^{2}}{w} d z+\frac{1}{2}\left(a^{3}+\frac{1}{a^{3}}\right) \int_{\gamma} \frac{z^{6}}{w^{3}} d z, \\
\int_{\gamma} \frac{d z}{w^{3}} & =\frac{2}{3}\left(a^{3}+\frac{1}{a^{3}}\right) \int_{\gamma} \frac{z^{2}}{w} d z+\left(2 a^{6}+\frac{2}{a^{6}}-3\right) \int_{\gamma} \frac{z^{6}}{w^{3}} d z, \\
\int_{\gamma} \frac{z^{7}}{w^{3}} d z & =\frac{1}{6} \int_{\gamma} \frac{d z}{w}+\frac{1}{2}\left(a^{3}+\frac{1}{a^{3}}\right) \int_{\gamma} \frac{z^{4}}{w^{3}} d z, \\
\int_{\gamma} \frac{z^{8}}{w^{3}} d z & =\int_{\gamma} \frac{z^{2}}{w^{3}} d z, \\
\int_{\gamma} \frac{z^{9}}{w^{3}} d z & =\frac{5}{6} \int_{\gamma} \frac{z^{2}}{w} d z+\frac{1}{2}\left(a^{3}+\frac{1}{a^{3}}\right) \int_{\gamma} \frac{z^{6}}{w^{3}} d z .
\end{aligned}
$$

Proof. Substituting $\alpha=1, \beta=-1$ to (3.1) yields (3.3). Substituting $\alpha=2$, $\beta=-1$ to (3.1) gives (3.4). Substituting $\alpha=3, \beta=-1$ (3.1) implies (3.5). Substituting $\alpha=0, \beta=-1$ to (3.2) yields

$$
\int_{\gamma} \frac{d z}{w^{3}}=4\left(a^{3}+\frac{1}{a^{3}}\right) \int_{\gamma} \frac{z^{3}}{w^{3}} d z-7 \int_{\gamma} \frac{z^{6}}{w^{3}} d z
$$




$$
\underbrace{=}_{\text {[3.5) }} \frac{2}{3}\left(a^{3}+\frac{1}{a^{3}}\right) \int_{\gamma} \frac{z^{2}}{w} d z+\left(2 a^{6}+\frac{2}{a^{6}}-3\right) \int_{\gamma} \frac{z^{6}}{w^{3}} d z
$$

Substituting $\alpha=1, \beta=-1$ to (3.2) gives

$$
\begin{aligned}
& \int_{\gamma} \frac{z^{7}}{w^{3}} d z=\frac{1}{5} \int_{\gamma} \frac{z}{w^{3}} d z+\frac{2}{5}\left(a^{3}+\frac{1}{a^{3}}\right) \int_{\gamma} \frac{z^{4}}{w^{3}} d z \\
& \underbrace{=}_{(3.3)} \frac{1}{6} \int_{\gamma} \frac{d z}{w}+\frac{1}{2}\left(a^{3}+\frac{1}{a^{3}}\right) \int_{\gamma} \frac{z^{4}}{w^{3}} d z .
\end{aligned}
$$

Substituting $\alpha=2, \beta=-1$ to (3.2) implies (3.8). Finally, we have

$$
\begin{aligned}
& \int_{\gamma} \frac{z^{9}}{w^{3}} d z=\int_{\gamma} \frac{z^{2}}{w^{3}}\left\{w^{2}+\left(a^{3}+\frac{1}{a^{3}}\right) z^{4}-z\right\} d z \\
&=\int_{\gamma} \frac{z^{2}}{w} d z+\left(a^{3}+\frac{1}{a^{3}}\right) \int_{\gamma} \frac{z^{6}}{w^{3}} d z-\int_{\gamma} \frac{z^{3}}{w^{3}} d z \\
& \underbrace{=}_{(3.5)} \frac{5}{6} \int_{\gamma} \frac{z^{2}}{w} d z+\frac{1}{2}\left(a^{3}+\frac{1}{a^{3}}\right) \int_{\gamma} \frac{z^{6}}{w^{3}} d z .
\end{aligned}
$$

Next we consider the basis of the tangent space of the complex Lagrangian cone via the complex period matrix. First we consider deformations of the complex structure of $M$. Let $a_{1}, a_{2}, \ldots, a_{6}$ be six points in $\mathbb{C} \backslash\{0\}$ satisfying

$$
w^{2}=z\left(z^{3}-a^{3}\right)\left(z^{3}-\frac{1}{a^{3}}\right)=z\left(z-a_{1}\right)\left(z-a_{2}\right) \cdots\left(z-a_{6}\right) .
$$

We now deform $M$ as follows: $w^{2}=z\left(z-z_{1}\right)\left(z-z_{2}\right) \cdots\left(z-z_{6}\right)$. Since

$\left.2 w \frac{\partial w}{\partial z_{i}}\right|_{\left(z_{1}, \ldots, z_{6}\right)=\left(a_{1}, \ldots, a_{6}\right)}=-z\left(z-a_{1}\right) \cdots\left(z-a_{i-1}\right)\left(z-a_{i+1}\right) \cdots\left(z-a_{6}\right)=-\frac{w^{2}}{z-a_{i}}$,

we have

$\left.\frac{\partial}{\partial z_{i}}\right|_{\left(z_{1}, \ldots, z_{6}\right)=\left(a_{1}, \ldots, a_{6}\right)} \int_{\gamma} \frac{1-z^{2}}{w} d z=\left.\int_{\gamma} \frac{\partial}{\partial z_{i}}\right|_{z=a_{i}} \frac{1-z^{2}}{w} d z=\frac{1}{2} \int_{\gamma} \frac{1-z^{2}}{w\left(z-a_{i}\right)} d z$

for an arbitrary 1-cycle $\gamma$. Applying the same technique, we find

$$
\left.\frac{\partial}{\partial z_{i}}\right|_{\left(z_{1}, \ldots, z_{6}\right)=\left(a_{1}, \ldots, a_{6}\right)}\left(\int_{A_{1}}\left(\begin{array}{c}
\frac{1-z^{2}}{w} d z \\
\frac{i\left(1+z^{2}\right)}{w} d z \\
\frac{2 z}{w} d z
\end{array}\right), \ldots, \int_{B_{3}}\left(\begin{array}{c}
\frac{1-z^{2}}{w} d z \\
\frac{i\left(1+z^{2}\right)}{w} d z \\
\frac{2 z}{w} d z
\end{array}\right)\right)
$$




$$
=\frac{1}{2}\left(\int_{A_{1}}\left(\begin{array}{c}
\frac{1-z^{2}}{w\left(z-a_{i}\right)} d z \\
\frac{i\left(1+z^{2}\right)}{w\left(z-a_{i}\right)} d z \\
\frac{2 z}{w\left(z-a_{i}\right)} d z
\end{array}\right), \ldots, \int_{B_{3}}\left(\begin{array}{l}
\frac{1-z^{2}}{w\left(z-a_{i}\right)} d z \\
\frac{i\left(1+z^{2}\right)}{w\left(z-a_{i}\right)} d z \\
\frac{2 z}{w\left(z-a_{i}\right)} d z
\end{array}\right)\right) .
$$

To describe

$$
\int_{\gamma} \frac{1-z^{2}}{w\left(z-a_{i}\right)} d z, \quad \int_{\gamma} \frac{i\left(1+z^{2}\right)}{w\left(z-a_{i}\right)} d z, \quad \int_{\gamma} \frac{2 z}{w\left(z-a_{i}\right)} d z
$$

via periods of the abelian differentials of the second kind, we set

$$
\begin{aligned}
w^{2} & =z\left(z-a_{i}\right)\left(z^{5}+\alpha_{i 4} z^{4}+\alpha_{i 3} z^{3}+\alpha_{i 2} z^{2}+\alpha_{i 1} z+\alpha_{i 0}\right) \\
& =z\left(z^{3}-a^{3}\right)\left(z^{3}-\frac{1}{a^{3}}\right)
\end{aligned}
$$

that is,

$$
\alpha_{i 0}=-\frac{1}{a_{i}}, \quad \alpha_{i 1}=-\frac{1}{a_{i}^{2}}, \alpha_{i 2}=-\frac{1}{a_{i}^{3}}, \quad \alpha_{i 3}=a_{i}^{2}, \quad \alpha_{i 4}=a_{i} .
$$

By Lemma 3.2, we have

$$
\begin{aligned}
& \int_{\gamma} \frac{d z}{w\left(z-a_{i}\right)}=\int_{\gamma} \frac{1}{w^{3}} z\left(z^{5}+\alpha_{i 4} z^{4}+\alpha_{i 3} z^{3}+\alpha_{i 2} z^{2}+\alpha_{i 1} z+\alpha_{i 0}\right) d z \\
& =\frac{5}{6} \alpha_{i 0} \int_{\gamma} \frac{d z}{w}+\frac{1}{2} \alpha_{i 1} \int_{\gamma} \frac{z}{w} d z+\frac{1}{6} \alpha_{i 2} \int_{\gamma} \frac{z^{2}}{w} d z \\
& +\left\{\frac{\alpha_{i 0}}{2}\left(a^{3}+\frac{1}{a^{3}}\right)+\alpha_{i 3}\right\} \int_{\gamma} \frac{z^{4}}{w^{3}} d z+\left\{\frac{\alpha_{i 1}}{2}\left(a^{3}+\frac{1}{a^{3}}\right)+\alpha_{i 4}\right\}_{\gamma} \frac{z^{5}}{w^{3}} d z \\
& +\left\{\frac{\alpha_{i 2}}{2}\left(a^{3}+\frac{1}{a^{3}}\right)+1\right\} \int_{\gamma} \frac{z^{6}}{w^{3}} d z \\
& \int_{\gamma} \frac{z}{w\left(z-a_{i}\right)} d z=\frac{1}{6} \int_{\gamma} \frac{d z}{w}+\frac{1}{2} \alpha_{i 0} \int_{\gamma} \frac{z}{w} d z+\frac{1}{6} \alpha_{i 1} \int_{\gamma} \frac{z^{2}}{w} d z \\
& +\left\{\frac{1}{2}\left(a^{3}+\frac{1}{a^{3}}\right)+\alpha_{i 2}\right\} \int_{\gamma} \frac{z^{4}}{w^{3}} d z+\left\{\frac{\alpha_{i 0}}{2}\left(a^{3}+\frac{1}{a^{3}}\right)+\alpha_{i 3}\right\} \int_{\gamma} \frac{z^{5}}{w^{3}} d z \\
& +\left\{\frac{\alpha_{i 1}}{2}\left(a^{3}+\frac{1}{a^{3}}\right)+\alpha_{i 4}\right\} \int_{\gamma} \frac{z^{6}}{w^{3}} d z, \\
& \int_{\gamma} \frac{z^{2}}{w\left(z-a_{i}\right)} d z=\frac{1}{6} \alpha_{i 4} \int_{\gamma} \frac{d z}{w}+\frac{1}{2} \int_{\gamma} \frac{z}{w} d z+\frac{1}{6} \alpha_{i 0} \int_{\gamma} \frac{z^{2}}{w} d z \\
& +\left\{\frac{\alpha_{i 4}}{2}\left(a^{3}+\frac{1}{a^{3}}\right)+\alpha_{i 1}\right\} \int_{\gamma} \frac{z^{4}}{w^{3}} d z+\left\{\frac{1}{2}\left(a^{3}+\frac{1}{a^{3}}\right)+\alpha_{i 2}\right\} \int_{\gamma} \frac{z^{5}}{w^{3}} d z \\
& +\left\{\frac{\alpha_{i 0}}{2}\left(a^{3}+\frac{1}{a^{3}}\right)+\alpha_{i 3}\right\} \int_{\gamma} \frac{z^{6}}{w^{3}} d z .
\end{aligned}
$$


Thus, setting

$$
P_{1}=\left(\begin{array}{ccc}
1 & 0 & -1 \\
i & 0 & i \\
0 & 2 & 0
\end{array}\right), \quad P_{2}=\left(\begin{array}{cccccc}
\frac{1}{2} & -\frac{i}{2} & 0 & 0 & 0 & 0 \\
0 & 0 & \frac{1}{2} & 0 & 0 & 0 \\
-\frac{1}{2} & -\frac{i}{2} & 0 & 0 & 0 & 0 \\
0 & 0 & 0 & \frac{1}{2} & -\frac{i}{2} & 0 \\
0 & 0 & 0 & 0 & 0 & 1 \\
0 & 0 & 0 & -\frac{1}{2} & -\frac{i}{2} & 0
\end{array}\right)
$$

and

$$
P_{a_{i}}=\left(\begin{array}{cccccc}
-\frac{5}{6 a_{i}} & -\frac{1}{2 a_{i}^{2}} & -\frac{1}{6 a_{i}^{3}} & \frac{1}{2}\left(a_{i}^{2}-\frac{1}{a_{i}^{4}}\right) & \frac{1}{2}\left(a_{i}-\frac{1}{a_{i}^{5}}\right) & \frac{1}{2}\left(1-\frac{1}{a_{i}^{6}}\right) \\
\frac{1}{6} & -\frac{1}{2 a_{i}} & -\frac{1}{6 a_{i}^{2}} & \frac{1}{2}\left(a_{i}^{3}-\frac{1}{a_{i}^{3}}\right) & \frac{1}{2}\left(a_{i}^{2}-\frac{1}{a_{i}^{4}}\right) & \frac{1}{2}\left(a_{i}-\frac{1}{a_{i}^{5}}\right) \\
\frac{a_{i}}{6} & \frac{1}{2} & -\frac{1}{6 a_{i}} & \frac{1}{2}\left(a_{i}^{4}-\frac{1}{a_{i}^{2}}\right) & \frac{1}{2}\left(a_{i}^{3}-\frac{1}{a_{i}^{3}}\right) & \frac{1}{2}\left(a_{i}^{2}-\frac{1}{a_{i}^{4}}\right)
\end{array}\right),
$$

we find

$$
\left(\begin{array}{c}
\int_{\gamma} \frac{1-z^{2}}{w\left(z-a_{i}\right)} d z \\
\int_{\gamma} \frac{i\left(1+z^{2}\right)}{w\left(z-a_{i}\right)} d z \\
\int_{\gamma} \frac{2 z}{w\left(z-a_{i}\right)} d z
\end{array}\right)=P_{1} P_{a_{i}} P_{2}\left(\begin{array}{c}
\int_{\gamma} \frac{1-z^{2}}{w} d z \\
\int_{\gamma} \frac{i\left(1+z^{2}\right)}{w} d z \\
\int_{\gamma} \frac{2 z}{w} d z \\
\int_{\gamma} \frac{z^{4}-z^{6}}{w^{3}} d z \\
\int_{\gamma} \frac{i\left(z^{4}+z^{6}\right)}{w^{3}} d z \\
\int_{\gamma} \frac{z^{5}}{w^{3}} d z
\end{array}\right)
$$

Let $\Omega_{\mathrm{H}}$ be the complex period matrix of the abelian differentials of the second kind (see $\S$ 5.11), that is,

$$
i\left(\begin{array}{cccccc}
0 & \frac{\sqrt{3}}{2}(A+i B) & 0 & -\sqrt{3} A & -2 \sqrt{3} A & -\sqrt{3} A \\
2 A & \frac{-3 A+i B}{2} & A-i B & i B & 0 & A \\
-i D & -C & 2 C+i D & C & 0 & i D \\
0 & -\frac{\sqrt{3}}{2}(E+i F) & 0 & \sqrt{3} E & 2 \sqrt{3} E & \sqrt{3} E \\
-2 E & \frac{3 E-i F}{2} & -E+i F & -i F & 0 & -E \\
i I & H & -2 H-i I & -H & 0 & -i I
\end{array}\right) .
$$

Then, choosing $a_{1}=a, a_{2}=e^{\frac{2}{3} \pi i} a, a_{3}=e^{\frac{4}{3} \pi i} a, a_{4}=1 / a$, and $a_{5}=e^{\frac{2}{3} \pi i} / a$, we obtain five tangent vectors $\left\{T_{i}\right\}_{i=1}^{5}:=\left\{\frac{1}{2} P_{1} P_{a_{i}} P_{2} \Omega_{\mathrm{H}}\right\}_{i=1}^{5}$ in $K_{3,6}$. Moreover, 
we shall consider the tangent vectors via an action of $S O(3, \mathbb{C}) \times(\mathbb{C} \backslash\{0\})$. Setting $C_{1}$ and $C_{2}$ are complex matrices of degree 3 given by

$$
\left(C_{1}, C_{2}\right)=i\left(\begin{array}{cccccc}
0 & \frac{\sqrt{3}}{2}(A+i B) & 0 & -\sqrt{3} A & -2 \sqrt{3} A & -\sqrt{3} A \\
2 A & \frac{-3 A+i B}{2} & A-i B & i B & 0 & A \\
-i D & -C & 2 C+i D & C & 0 & i D
\end{array}\right)
$$

we have the Riemann matrix $\tau=C_{1}^{-1} C_{2}$ and the following four tangent vectors in $K_{3,6}$ :

$$
\begin{aligned}
& T_{6}:=\left(C_{1}, C_{2}\right), \quad T_{7}:=\left(\begin{array}{ccc}
0 & 1 & 0 \\
-1 & 0 & 0 \\
0 & 0 & 0
\end{array}\right)\left(C_{1}, C_{2}\right), \\
& T_{8}:=\left(\begin{array}{ccc}
0 & 0 & 1 \\
0 & 0 & 0 \\
-1 & 0 & 0
\end{array}\right)\left(C_{1}, C_{2}\right), \quad T_{9}:=\left(\begin{array}{ccc}
0 & 0 & 0 \\
0 & 0 & 1 \\
0 & -1 & 0
\end{array}\right)\left(C_{1}, C_{2}\right) .
\end{aligned}
$$

\section{$3.2 \quad \mathrm{rPD}$ family}

We begin by giving some lemmas. Choosing $i z, e^{-\frac{\pi}{4} i} w$, and $i a$ instead of $z$, $w$, and $a$ in Lemma 3.1 and Lemma 3.2 respectively, we find the following two Lemmas.

\section{Lemma 3.3.}

$$
\begin{aligned}
& d\left(z^{\alpha} w^{\beta}\right)=\frac{z^{\alpha-1} w^{\beta-2}}{2}\left\{(2 \alpha+7 \beta) w^{2}+3 \beta\left(a^{3}-\frac{1}{a^{3}}\right) z^{4}+6 \beta z\right\} d z \\
& =\frac{z^{\alpha} w^{\beta-2}}{2}\left\{(2 \alpha+7 \beta) z^{6}+2(\alpha+2 \beta)\left(-a^{3}+\frac{1}{a^{3}}\right) z^{3}-(2 \alpha+\beta)\right\} d z
\end{aligned}
$$

Lemma 3.4. For an arbitrary 1-cycle $\gamma$, we have

$$
\begin{aligned}
\int_{\gamma} \frac{z}{w^{3}} d z & =-\frac{5}{6} \int_{\gamma} \frac{d z}{w}+\frac{1}{2}\left(-a^{3}+\frac{1}{a^{3}}\right) \int_{\gamma} \frac{z^{4}}{w^{3}} d z \\
\int_{\gamma} \frac{z^{2}}{w^{3}} d z & =-\frac{1}{2} \int_{\gamma} \frac{z}{w} d z+\frac{1}{2}\left(-a^{3}+\frac{1}{a^{3}}\right) \int_{\gamma} \frac{z^{5}}{w^{3}} d z \\
\int_{\gamma} \frac{z^{3}}{w^{3}} d z & =-\frac{1}{6} \int_{\gamma} \frac{z^{2}}{w} d z+\frac{1}{2}\left(-a^{3}+\frac{1}{a^{3}}\right) \int_{\gamma} \frac{z^{6}}{w^{3}} d z, \\
\int_{\gamma} \frac{d z}{w^{3}} & =\frac{2}{3}\left(a^{3}-\frac{1}{a^{3}}\right) \int_{\gamma} \frac{z^{2}}{w} d z+\left(2 a^{6}+\frac{2}{a^{6}}+3\right) \int_{\gamma} \frac{z^{6}}{w^{3}} d z, \\
\int_{\gamma} \frac{z^{7}}{w^{3}} d z & =\frac{1}{6} \int_{\gamma} \frac{d z}{w}+\frac{1}{2}\left(a^{3}-\frac{1}{a^{3}}\right) \int_{\gamma} \frac{z^{4}}{w^{3}} d z \\
\int_{\gamma} \frac{z^{8}}{w^{3}} d z & =-\int_{\gamma} \frac{z^{2}}{w^{3}} d z
\end{aligned}
$$




$$
\int_{\gamma} \frac{z^{9}}{w^{3}} d z=\frac{5}{6} \int_{\gamma} \frac{z^{2}}{w} d z+\frac{1}{2}\left(a^{3}-\frac{1}{a^{3}}\right) \int_{\gamma} \frac{z^{6}}{w^{3}} d z
$$

Next we consider the basis of the tangent space of the complex Lagrangian cone via the complex period matrix. First we consider deformations of the complex structure of $M$. Let $a_{1}, a_{2}, \ldots, a_{6}$ be six points in $\mathbb{C} \backslash\{0\}$ satisfying

$$
w^{2}=z\left(z^{3}-a^{3}\right)\left(z^{3}+\frac{1}{a^{3}}\right)=z\left(z-a_{1}\right)\left(z-a_{2}\right) \cdots\left(z-a_{6}\right) .
$$

We now deform $M$ as follows: $w^{2}=z\left(z-z_{1}\right)\left(z-z_{2}\right) \cdots\left(z-z_{6}\right)$. Then, for an arbitrary 1-cycle $\gamma$, we find

$$
\begin{aligned}
& \left.\frac{\partial}{\partial z_{i}}\right|_{\left(z_{1}, \ldots, z_{6}\right)=\left(a_{1}, \ldots, a_{6}\right)}\left(\int_{A_{1}}\left(\begin{array}{c}
\frac{1-z^{2}}{w} d z \\
\frac{i\left(1+z^{2}\right)}{w} d z \\
\frac{2 z}{w} d z
\end{array}\right), \ldots, \int_{B_{3}}\left(\begin{array}{c}
\frac{1-z^{2}}{w} d z \\
\frac{i\left(1+z^{2}\right)}{w} d z \\
\frac{2 z}{w} d z
\end{array}\right)\right) \\
& =\frac{1}{2}\left(\int_{A_{1}}\left(\begin{array}{l}
\frac{1-z^{2}}{w\left(z-a_{i}\right)} d z \\
\frac{i\left(1+z^{2}\right)}{w\left(z-a_{i}\right)} d z \\
\frac{2 z}{w\left(z-a_{i}\right)} d z
\end{array}\right), \ldots, \int_{B_{3}}\left(\begin{array}{l}
\frac{1-z^{2}}{w\left(z-a_{i}\right)} d z \\
\frac{i\left(1+z^{2}\right)}{w\left(z-a_{i}\right)} d z \\
\frac{2 z}{w\left(z-a_{i}\right)} d z
\end{array}\right)\right)
\end{aligned}
$$

To describe

$$
\int_{\gamma} \frac{1-z^{2}}{w\left(z-a_{i}\right)} d z, \quad \int_{\gamma} \frac{i\left(1+z^{2}\right)}{w\left(z-a_{i}\right)} d z, \quad \int_{\gamma} \frac{2 z}{w\left(z-a_{i}\right)} d z
$$

via periods of the abelian differentials of the second kind, we set

$w^{2}=z\left(z-a_{i}\right)\left(z^{5}+\alpha_{i 4} z^{4}+\alpha_{i 3} z^{3}+\alpha_{i 2} z^{2}+\alpha_{i 1} z+\alpha_{i 0}\right)=z\left(z^{3}-a^{3}\right)\left(z^{3}+\frac{1}{a^{3}}\right)$,

that is,

$$
\alpha_{i 0}=\frac{1}{a_{i}}, \quad \alpha_{i 1}=\frac{1}{a_{i}^{2}}, \alpha_{i 2}=\frac{1}{a_{i}^{3}}, \alpha_{i 3}=a_{i}^{2}, \alpha_{i 4}=a_{i} .
$$

Thus, setting

$$
P_{1}=\left(\begin{array}{ccc}
1 & 0 & -1 \\
i & 0 & i \\
0 & 2 & 0
\end{array}\right), \quad P_{2}=\left(\begin{array}{cccccc}
\frac{1}{2} & -\frac{i}{2} & 0 & 0 & 0 & 0 \\
0 & 0 & \frac{1}{2} & 0 & 0 & 0 \\
-\frac{1}{2} & -\frac{i}{2} & 0 & 0 & 0 & 0 \\
0 & 0 & 0 & \frac{1}{2} & -\frac{i}{2} & 0 \\
0 & 0 & 0 & 0 & 0 & 1 \\
0 & 0 & 0 & -\frac{1}{2} & -\frac{i}{2} & 0
\end{array}\right),
$$




$$
P_{a_{i}}=\left(\begin{array}{cccccc}
-\frac{5}{6 a_{i}} & -\frac{1}{2 a_{i}^{2}} & -\frac{1}{6 a_{i}^{3}} & \frac{1}{2}\left(a_{i}^{2}+\frac{1}{a_{i}^{4}}\right) & \frac{1}{2}\left(a_{i}+\frac{1}{a_{i}^{5}}\right) & \frac{1}{2}\left(1+\frac{1}{a_{i}^{6}}\right) \\
\frac{1}{6} & -\frac{1}{2 a_{i}} & -\frac{1}{6 a_{i}^{2}} & \frac{1}{2}\left(a_{i}^{3}+\frac{1}{a_{i}^{3}}\right) & \frac{1}{2}\left(a_{i}^{2}+\frac{1}{a_{i}^{4}}\right) & \frac{1}{2}\left(a_{i}+\frac{1}{a_{i}^{5}}\right) \\
\frac{a_{i}}{6} & \frac{1}{2} & -\frac{1}{6 a_{i}} & \frac{1}{2}\left(a_{i}^{4}+\frac{1}{a_{i}^{2}}\right) & \frac{1}{2}\left(a_{i}^{3}+\frac{1}{a_{i}^{3}}\right) & \frac{1}{2}\left(a_{i}^{2}+\frac{1}{a_{i}^{4}}\right)
\end{array}\right),
$$

we find

$$
\left(\begin{array}{l}
\int_{\gamma} \frac{1-z^{2}}{w\left(z-a_{i}\right)} d z \\
\int_{\gamma} \frac{i\left(1+z^{2}\right)}{w\left(z-a_{i}\right)} d z \\
\int_{\gamma} \frac{2 z}{w\left(z-a_{i}\right)} d z
\end{array}\right)=P_{1} P_{a_{i}} P_{2}\left(\begin{array}{c}
\int_{\gamma} \frac{1-z^{2}}{w} d z \\
\int_{\gamma} \frac{i\left(1+z^{2}\right)}{w} d z \\
\int_{\gamma} \frac{2 z}{w} d z \\
\int_{\gamma} \frac{z^{4}-z^{6}}{w^{3}} d z \\
\int_{\gamma} \frac{i\left(z^{4}+z^{6}\right)}{w^{3}} d z \\
\int_{\gamma} \frac{z^{5}}{w^{3}} d z
\end{array}\right)
$$

by Lemma 3.4 Let $\Omega_{\mathrm{rPD}}$ be the period matrix of the abelian differentials of the second kind (see $\S 5.2$ ), that is,

$$
i\left(\begin{array}{cccccc}
2 i B & -2(A+i B) & -(A+i B) & 2 A & 3(A-i B) & 2(A-i B) \\
-2 \sqrt{3} A & 0 & \sqrt{3}(A+i B) & -2 \sqrt{3} i B & \sqrt{3}(A-i B) & 0 \\
i D & C-i D & -C+i D & -C & 0 & -(C+i D) \\
-2 i F & 2(-E+i F) & -E+i F & 2 E & 3(E+i F) & 2(E+i F) \\
-2 \sqrt{3} E & 0 & \sqrt{3}(E-i F) & 2 \sqrt{3} i F & \sqrt{3}(E+i F) & 0 \\
i I & H-i I & -H+i I & -H & 0 & -(H+i I)
\end{array}\right) .
$$

Then, choosing $a_{1}=a, a_{2}=e^{\frac{2}{3} \pi i} a, a_{3}=e^{\frac{4}{3} \pi i} a, a_{4}=-1 / a$, and $a_{5}=-e^{\frac{2}{3} \pi i} / a$, we obtain five tangent vectors $\left\{T_{i}\right\}_{i=1}^{5}:=\left\{\frac{1}{2} P_{1} P_{a_{i}} P_{2} \Omega_{\mathrm{rPD}}\right\}_{i=1}^{5}$ in $K_{3,6}$. Moreover, we shall consider the tangent vectors via an action of $S O(3, \mathbb{C}) \times(\mathbb{C} \backslash\{0\})$. Setting $C_{1}$ and $C_{2}$ are complex matrices of degree 3 given by

$$
\left(C_{1}, C_{2}\right)=i\left(\begin{array}{cccccc}
2 i B & -2(A+i B) & -(A+i B) & 2 A & 3(A-i B) & 2(A-i B) \\
-2 \sqrt{3} A & 0 & \sqrt{3}(A+i B) & -2 \sqrt{3} i B & \sqrt{3}(A-i B) & 0 \\
i D & C-i D & -C+i D & -C & 0 & -(C+i D)
\end{array}\right),
$$

we have the Riemann matrix $\tau=C_{1}^{-1} C_{2}$ and the following four tangent vectors in $K_{3,6}$ :

$$
\begin{aligned}
& T_{6}:=\left(C_{1}, C_{2}\right), T_{7}:=\left(\begin{array}{ccc}
0 & 1 & 0 \\
-1 & 0 & 0 \\
0 & 0 & 0
\end{array}\right)\left(C_{1}, C_{2}\right), \\
& T_{8}:=\left(\begin{array}{ccc}
0 & 0 & 1 \\
0 & 0 & 0 \\
-1 & 0 & 0
\end{array}\right)\left(C_{1}, C_{2}\right), \quad T_{9}:=\left(\begin{array}{ccc}
0 & 0 & 0 \\
0 & 0 & 1 \\
0 & -1 & 0
\end{array}\right)\left(C_{1}, C_{2}\right) .
\end{aligned}
$$




\section{3 tP family, tD family}

Recall that tD family is parametrized by $a \in(-\infty,-2)$ instead of $a \in(2, \infty)$. Using the reparametrization $(u, v)=\left(e^{-\frac{\pi}{4} i} z, w\right)$, we transform tD family to a family of conjugate surfaces of minimal surfaces which belong to tP family. Note that the Morse index and the nullity of a minimal surface depend only on its Gauss map (see for instance [8]), and the signature of a minimal surface coincides with that of the associated surface (Theorem 9.1 in [3]). Hence the Morse index of a minimal surface is equal to that of its conjugate surface and so are the nullity and the signature. Thus we treat only tP family.

We begin by giving some lemmas.

\section{Lemma 3.5.}

$$
\begin{aligned}
d\left(z^{\alpha} w^{\beta}\right) & =z^{\alpha-1} w^{\beta-2}\left\{(\alpha+4 \beta) z^{8}+a(\alpha+2 \beta) z^{4}+\alpha\right\} d z \\
& =z^{\alpha-1} w^{\beta-2}\left\{(\alpha+4 \beta) w^{2}-2 a \beta z^{4}-4 \beta\right\} d z .
\end{aligned}
$$

Proof. Straightforward calculation.

Lemma 3.6. For an arbitrary 1-cycle $\gamma$, we have

$$
\begin{aligned}
\int_{\gamma} \frac{d z}{w^{3}} & =\frac{3}{4} \int_{\gamma} \frac{d z}{w}-\frac{a}{2} \int_{\gamma} z^{4} \frac{d z}{w^{3}}, \\
\int_{\gamma} z \frac{d z}{w^{3}} & =\frac{1}{2} \int_{\gamma} z \frac{d z}{w}-\frac{a}{2} \int_{\gamma} z^{5} \frac{d z}{w^{3}}, \\
\int_{\gamma} z^{2} \frac{d z}{w^{3}} & =\frac{1}{4} \int_{\gamma} z^{2} \frac{d z}{w}-\frac{a}{2} \int_{\gamma} z^{6} \frac{d z}{w^{3}}, \\
\int_{\gamma} z^{3} \frac{d z}{w^{3}} & =\int_{\gamma} z^{7} \frac{d z}{w^{3}}=0 \\
\int_{\gamma} z^{8} \frac{d z}{w^{3}} & =\frac{1}{4} \int_{\gamma} \frac{d z}{w}-\frac{a}{2} \int_{\gamma} z^{4} \frac{d z}{w^{3}}, \\
\int_{\gamma} z^{9} \frac{d z}{w^{3}} & =\int_{\gamma} z \frac{d z}{w^{3}}, \\
\int_{\gamma} z^{10} \frac{d z}{w^{3}} & =\frac{3}{4} \int_{\gamma} z^{2}, \frac{d z}{w}-\frac{a}{2} \int_{\gamma} z^{6} \frac{d z}{w^{3}} .
\end{aligned}
$$

Proof. Substituting $\alpha=1, \beta=-1$ to (3.20) yields (3.21). Substituting $\alpha=2$, $\beta=-1$ to (3.20) gives (3.22). Substituting $\alpha=3, \beta=-1$ (3.20) implies (3.23). Substituting $(\alpha, \beta)=(0,-1),(4,-1)$ to (3.19) yields (3.24). Substituting $\alpha=$ $1, \beta=-1$ to (3.19) and (3.21) give (3.25). Substituting $\alpha=2, \beta=-1$ to (3.19) implies (3.26). Combining the equation $w^{2}=z^{8}+a z^{4}+1$ and (3.23) yields (3.27).

Next we consider the basis of the tangent space of the complex Lagrangian cone via the complex period matrix. First we consider deformations of the 
complex structure of $M$. We assume $a \in(2, \infty)$. Let $a_{1}, a_{2}, \ldots, a_{8}$ be eight points in $\mathbb{C} \backslash\{0\}$ :

$$
w^{2}=z^{8}+a z^{4}+1=\left(z-a_{1}\right)\left(z-a_{2}\right) \cdots\left(z-a_{8}\right) .
$$

We now deform $M$ as follows: $w^{2}=\left(z-z_{1}\right)\left(z-z_{2}\right) \cdots\left(z-z_{8}\right)$. Then, for an arbitrary 1-cycle $\gamma$, we find

$$
\begin{aligned}
& \left.\frac{\partial}{\partial z_{i}}\right|_{\left(z_{1}, \ldots, z_{8}\right)=\left(a_{1}, \ldots, a_{8}\right)}\left(\int_{A_{1}}\left(\begin{array}{c}
\frac{1-z^{2}}{w} d z \\
\frac{i\left(1+z^{2}\right)}{w} d z \\
\frac{2 z}{w} d z
\end{array}\right), \ldots, \int_{B_{3}}\left(\begin{array}{c}
\frac{1-z^{2}}{w} d z \\
\frac{i\left(1+z^{2}\right)}{w} d z \\
\frac{2 z}{w} d z
\end{array}\right)\right) \\
& =\frac{1}{2}\left(\int_{A_{1}}\left(\begin{array}{l}
\frac{1-z^{2}}{w\left(z-a_{i}\right)} d z \\
\frac{i\left(1+z^{2}\right)}{w\left(z-a_{i}\right)} d z \\
\frac{2 z}{w\left(z-a_{i}\right)} d z
\end{array}\right), \ldots, \int_{B_{3}}\left(\begin{array}{l}
\frac{1-z^{2}}{w\left(z-a_{i}\right)} d z \\
\frac{i\left(1+z^{2}\right)}{w\left(z-a_{i}\right)} d z \\
\frac{2 z}{w\left(z-a_{i}\right)} d z
\end{array}\right)\right)
\end{aligned}
$$

To describe

$$
\int_{\gamma} \frac{1-z^{2}}{w\left(z-a_{i}\right)} d z, \quad \int_{\gamma} \frac{i\left(1+z^{2}\right)}{w\left(z-a_{i}\right)} d z, \quad \int_{\gamma} \frac{2 z}{w\left(z-a_{i}\right)} d z
$$

via periods of the abelian differentials of the second kind, we set

$$
\begin{aligned}
w^{2} & =\left(z-a_{i}\right)\left(z^{7}+\alpha_{i 6} z^{6}+\alpha_{i 5} z^{5}+\alpha_{i 4} z^{4}+\alpha_{i 3} z^{3}+\alpha_{i 2} z^{2}+\alpha_{i 1} z+\alpha_{i 0}\right) \\
& =z^{8}+a z^{4}+1
\end{aligned}
$$

that is,

$$
\begin{aligned}
& \alpha_{i 0}=-\frac{1}{a_{i}}, \quad \alpha_{i 1}=-\frac{1}{a_{i}^{2}}, \quad \alpha_{i 2}=-\frac{1}{a_{i}^{3}}, \alpha_{i 3}=-\frac{1}{a_{i}^{4}}, \\
& \alpha_{i 4}=a_{i}^{3}, \quad \alpha_{i 5}=a_{i}^{2}, \quad \alpha_{i 6}=a_{i} .
\end{aligned}
$$

Thus, setting

$$
P_{1}=\left(\begin{array}{ccc}
1 & 0 & -1 \\
i & 0 & i \\
0 & 2 & 0
\end{array}\right), \quad P_{2}=\left(\begin{array}{cccccc}
\frac{1}{2} & -\frac{i}{2} & 0 & 0 & 0 & 0 \\
0 & 0 & \frac{1}{2} & 0 & 0 & 0 \\
-\frac{1}{2} & -\frac{i}{2} & 0 & 0 & 0 & 0 \\
0 & 0 & 0 & \frac{1}{2} & -\frac{i}{2} & 0 \\
0 & 0 & 0 & 0 & 0 & 1 \\
0 & 0 & 0 & -\frac{1}{2} & -\frac{i}{2} & 0
\end{array}\right),
$$


and

$$
P_{a_{i}}=\left(\begin{array}{cccccc}
-\frac{3}{4 a_{i}} & -\frac{1}{2 a_{i}^{2}} & -\frac{1}{4 a_{i}^{3}} & \frac{a}{2 a_{i}}+a_{i}^{3} & \frac{a}{2 a_{i}^{2}}+a_{i}^{2} & \frac{a}{2 a_{i}^{3}}+a_{i} \\
\frac{1}{4} & -\frac{1}{2 a_{i}} & -\frac{1}{4 a_{i}^{2}} & -\frac{a}{2}-\frac{1}{a_{i}^{4}} & \frac{a}{2 a_{i}}+a_{i}^{3} & \frac{a}{2 a_{i}^{2}}+a_{i}^{2} \\
\frac{a_{i}}{4} & \frac{1}{2} & -\frac{1}{4 a_{i}} & -\frac{a}{2} a_{i}-\frac{1}{a_{i}^{3}} & -\frac{a}{2}-\frac{1}{a_{i}^{4}} & \frac{a}{2 a_{i}}+a_{i}^{3}
\end{array}\right),
$$

we find

$$
\left(\begin{array}{c}
\int_{\gamma} \frac{1-z^{2}}{w\left(z-a_{i}\right)} d z \\
\int_{\gamma} \frac{i\left(1+z^{2}\right)}{w\left(z-a_{i}\right)} d z \\
\int_{\gamma} \frac{2 z}{w\left(z-a_{i}\right)} d z
\end{array}\right)=P_{1} P_{a_{i}} P_{2}\left(\begin{array}{c}
\int_{\gamma} \frac{1-z^{2}}{w} d z \\
\int_{\gamma} \frac{i\left(1+z^{2}\right)}{w} d z \\
\int_{\gamma} \frac{2 z}{w} d z \\
\int_{\gamma} \frac{z^{4}-z^{6}}{w^{3}} d z \\
\int_{\gamma} \frac{i\left(z^{4}+z^{6}\right)}{w^{3}} d z \\
\int_{\gamma} \frac{z^{5}}{w^{3}} d z
\end{array}\right)
$$

by Lemma 3.6 Let $\Omega_{\mathrm{tP}}$ be the period matrix of the abelian differentials of the second kind (see $\S 5.3$ ), that is,

$$
\left(\begin{array}{cccccc}
-i B & -A & i B & -i B & -2 i B & -i B \\
A & i B & -A & i B & 0 & -i B \\
-i D & i D & -i D & C & 0 & C \\
-i F & -E & i F & -i F & -2 i F & -i F \\
E & i F & -E & i F & 0 & -i F \\
-i I & i I & -i I & H & 0 & H
\end{array}\right) .
$$

Set $\alpha:=\sqrt{\frac{\sqrt{a+2}+\sqrt{a-2}}{2}}>1$. Then, choosing $a_{1}=e^{\frac{\pi}{4} i} \alpha, a_{2}=e^{\frac{3}{4} \pi i} \alpha, a_{3}=$ $e^{-\frac{\pi}{4} i} \alpha, a_{4}=e^{-\frac{3}{4} \pi i} \alpha$, and $a_{5}=e^{\frac{\pi}{4} i} / \alpha$, we obtain five tangent vectors $\left\{T_{i}\right\}_{i=1}^{5}:=$ $\left\{\frac{1}{2} P_{1} P_{a_{i}} P_{2} \Omega_{\mathrm{tP}}\right\}_{i=1}^{5}$ in $K_{3,6}$. Moreover, we shall consider the tangent vectors via an action of $\bar{S} O(3, \mathbb{C}) \times(\mathbb{C} \backslash\{0\})$. Setting $C_{1}$ and $C_{2}$ are complex matrices of degree 3 given by

$$
\left(C_{1}, C_{2}\right)=\left(\begin{array}{cccccc}
-i B & -A & i B & -i B & -2 i B & -i B \\
A & i B & -A & i B & 0 & -i B \\
-i D & i D & -i D & C & 0 & C
\end{array}\right),
$$

we have the Riemann matrix $\tau=C_{1}^{-1} C_{2}$ and the following four tangent vectors in $K_{3,6}$ :

$$
T_{6}:=\left(C_{1}, C_{2}\right), \quad T_{7}:=\left(\begin{array}{ccc}
0 & 1 & 0 \\
-1 & 0 & 0 \\
0 & 0 & 0
\end{array}\right)\left(C_{1}, C_{2}\right)
$$




$$
T_{8}:=\left(\begin{array}{ccc}
0 & 0 & 1 \\
0 & 0 & 0 \\
-1 & 0 & 0
\end{array}\right)\left(C_{1}, C_{2}\right), \quad T_{9}:=\left(\begin{array}{ccc}
0 & 0 & 0 \\
0 & 0 & 1 \\
0 & -1 & 0
\end{array}\right)\left(C_{1}, C_{2}\right)
$$

\section{4 tCLP family}

Since $M$ is defined by the same equation as tP family, we can take the same $P_{1}$, $P_{a_{i}}$, and $P_{2}$ as in $\S 3.3$ for $a \in(-2,2)$. We now assume $a \in[0,2)$ because every minimal surface for $a \in(-2,0]$ can be transformed to its conjugate surface for $-a \in[0,2)$. Let $\Omega_{\mathrm{tCLP}}$ be the period matrix of the abelian differentials of the second kind (see $\S$ 5.4), that is,

$$
\left(\begin{array}{cccccc}
-i B & i B & i B & 0 & -A & -A \\
-i B & -i B & i B & A & A & 0 \\
-C & C & -C & -i D & 0 & -i D \\
-i F & i F & i F & 0 & E & E \\
-i F & -i F & i F & -E & -E & 0 \\
-H & H & -H & i I & 0 & i I
\end{array}\right)
$$

Set $e^{i \alpha}:=-\frac{a}{2}+i \frac{\sqrt{4-a^{2}}}{2} \in S^{1} \subset \mathbb{C}(\alpha \in[\pi / 2, \pi))$. Choosing $a_{1}=e^{\frac{\alpha}{4} i}, a_{2}=$ $i e^{\frac{\alpha}{4} i}, a_{3}=-e^{\frac{\alpha}{4} i}, a_{4}=-i e^{\frac{\alpha}{4} i}$, and $a_{5}=e^{-\frac{\alpha}{4} i}$, we obtain five tangent vectors $\left\{T_{i}\right\}_{i=1}^{5}:=\left\{\frac{1}{2} P_{1} P_{a_{i}} P_{2} \Omega_{\mathrm{tCLP}}\right\}_{i=1}^{5}$ in $K_{3,6}$. Moreover, setting $C_{1}$ and $C_{2}$ are complex matrices of degree 3 given by

$$
\left(C_{1}, C_{2}\right)=\left(\begin{array}{cccccc}
-i B & i B & i B & 0 & -A & -A \\
-i B & -i B & i B & A & A & 0 \\
-C & C & -C & -i D & 0 & -i D
\end{array}\right)
$$

we have the Riemann matrix $\tau=C_{1}^{-1} C_{2}$ and the following four tangent vectors in $K_{3,6}$ :

$$
\begin{aligned}
& T_{6}:=\left(C_{1}, C_{2}\right), T_{7}:=\left(\begin{array}{ccc}
0 & 1 & 0 \\
-1 & 0 & 0 \\
0 & 0 & 0
\end{array}\right)\left(C_{1}, C_{2}\right), \\
& T_{8}:=\left(\begin{array}{ccc}
0 & 0 & 1 \\
0 & 0 & 0 \\
-1 & 0 & 0
\end{array}\right)\left(C_{1}, C_{2}\right), \quad T_{9}:=\left(\begin{array}{ccc}
0 & 0 & 0 \\
0 & 0 & 1 \\
0 & -1 & 0
\end{array}\right)\left(C_{1}, C_{2}\right) .
\end{aligned}
$$

\section{Morse indices and nullities}

In this section, we will show our main results. Every family of minimal surfaces is parametrized by $a$ which belongs to a suitable interval. First, we find $\{a \mid \operatorname{det} W=0\}$. By these points, each domain interval can be divided into some intervals. After that, we shall consider the negative eigenvalues of $W_{2}-W_{1}$ on each divided interval. Our main results follow from the procedure. 
Remark 4.1. A minimal surface with inde $_{E}=0$ must be the totally geodesic subtorus. Thus every minimal surface in this paper satisfies inde $_{E} \geq 1$. Suppose that a family of minimal surfaces parametrized by $a \in(\alpha, \beta)$ satisfies $\operatorname{index}_{E}=1$. Then, by a fundamental eigenvalues argument, we have $i n d e x_{E}=1$ for $a=\alpha, \beta$. In fact, let

$$
\underbrace{\lambda_{1}, \cdots, \lambda_{k}}_{<0}, 0, \cdots, 0, \underbrace{\lambda_{k+1}, \cdots}_{>0}
$$

be eigenvalues of $E_{\phi}$ at $a=\alpha$. In a neighborhood of $a=\alpha$, we have index $_{E} \geq k$. If inde $_{E}=1$ on $(\alpha, \beta)$, then $k \leq 1$, that is, $k=1$ holds. So is the case $a=\beta$.

Since each $T_{j}, W, W_{j}$ are too complicated, we use numerical arguments by Mathematica.

\subsection{H family}

The curve $\operatorname{det} W$ may meet the real axis at two points $a_{1}, a_{2}$ (see Figure 4.1). Thus we consider three intervals $\left(0, a_{1}\right),\left(a_{1}, a_{2}\right),\left(a_{2}, 1\right)$. Sets of eigenvalues of
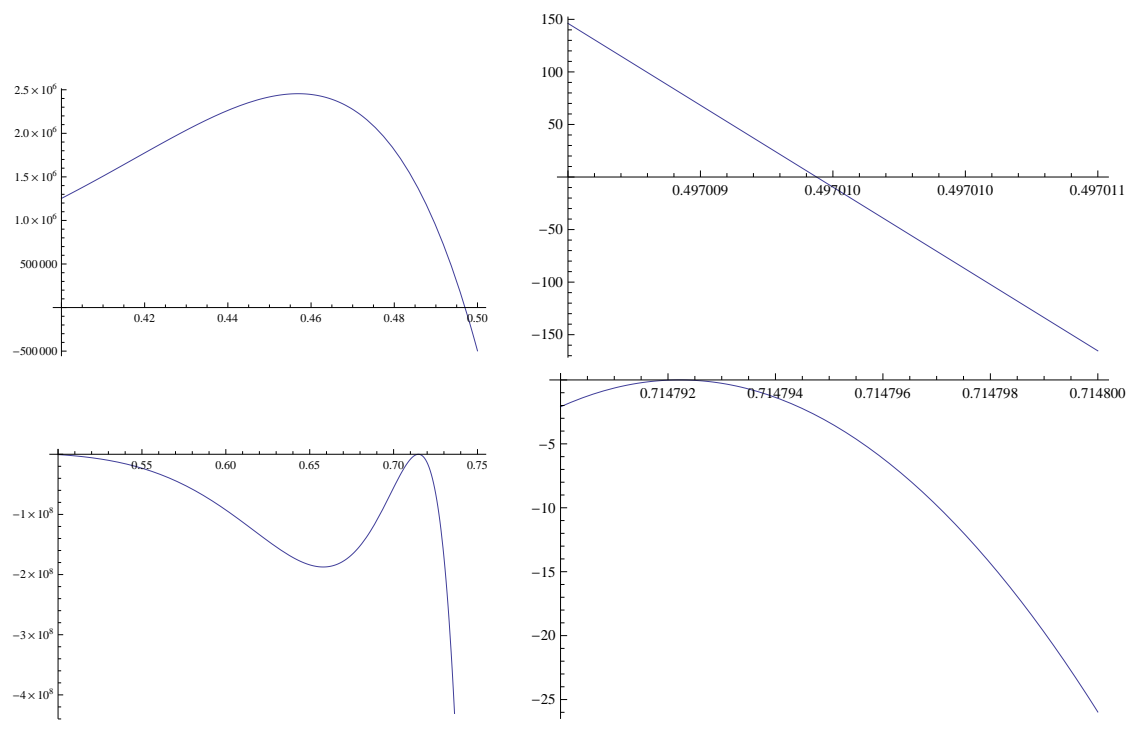

Figure 4.1: $\operatorname{det} W$ (the real axis is defined by $a$ )

$W$ for $a=0.49700993839805,0.714792215373045$ are given by

$$
\begin{aligned}
& \{160.732,92.2764,83.1167,71.3028,-4.65805,-3.62774,-0.180182, \\
& \quad-0.0540145,0\}, \\
& \{231.28,147.299,131.781,125.929,-7.19325,-6.91484,-5.16664, \\
& \quad 0,0\},
\end{aligned}
$$


respectively. Hence $a_{1} \approx 0.49701$ and $a_{2} \approx 0.71479$.

Substituting $a=0.3,0.5,0.8$ to $W$, we obtain sets of the eigenvalues as follows:

$$
\begin{aligned}
& \{144.683,53.6184,39.0519,34.6726,-7.31714,-2.4839,1.01569, \\
& \quad-0.0461945,-0.00828724\}, \\
& \{161.373,92.8348,83.8803,71.9439,-4.64655,-3.64618,-0.18237, \\
& \quad-0.0647386,-0.0274727\}, \\
& \{275.447,181.897,155.676,149.321,-14.6043,-12.4717,-8.58915, \\
& \quad 0.405355,0.108547\} .
\end{aligned}
$$

Also, substituting $a=0.3,0.5,0.8$ to $W_{2}-W_{1}$, we have the following sets of the eigenvalues:

$$
\begin{aligned}
& \{17.7972,17.7971,11.0343,11.0248,5.34828,-1.75067,0.119967,0.085154, \\
& \quad 0.0207832,0.014763,0,0,0,0,0,0,0,0\} \\
& \{14.8116,14.7502,10.5123,9.83969,9.68212,0.819204,0.324313,0.190585, \\
& \quad 0.0728135,0.050784,0,0,0,0,0,0,0,0\} \\
& \{44.9919,38.0399,36.0209,29.0891,24.1326,20.2568,3.46409,1.12237, \\
& \quad-0.384964,-0.130142,0,0,0,0,0,0,0,0\} .
\end{aligned}
$$

Therefore, we conclude that

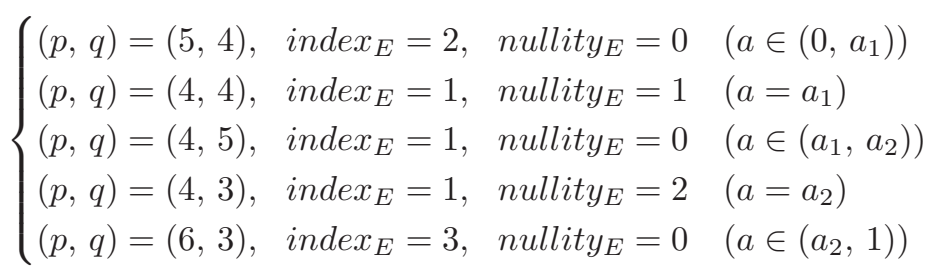

\section{2 rPD family}

The curve $\operatorname{det} W$ may meet the real axis at a point $a_{1}$ (see Figure 4.2). Thus we consider two intervals $\left(0, a_{1}\right),\left(a_{1}, 1\right]$. A set of eigenvalues of $W$ for $a=$ 0.494722327827355 is given by

$$
\begin{aligned}
& \{160.726,90.8344,81.34,70.8198,-4.32264,-3.35846,-0.218914, \\
& \quad-0.0654375,0\} .
\end{aligned}
$$

Hence $a_{1} \approx 0.494722$.

Substituting $a=0.3,0.5$ to $W$, we obtain sets of the eigenvalues as follows:

$$
\begin{aligned}
& \{144.749,53.5907,39.027,34.7134,-7.29118,-2.47246,1.01161, \\
& \quad-0.0471324,-0.00845948\}, \\
& \{161.851,91.747,82.5866,71.9293,-4.28067,-3.37296,-0.226271, \\
& \quad-0.0900042,-0.041516\}
\end{aligned}
$$



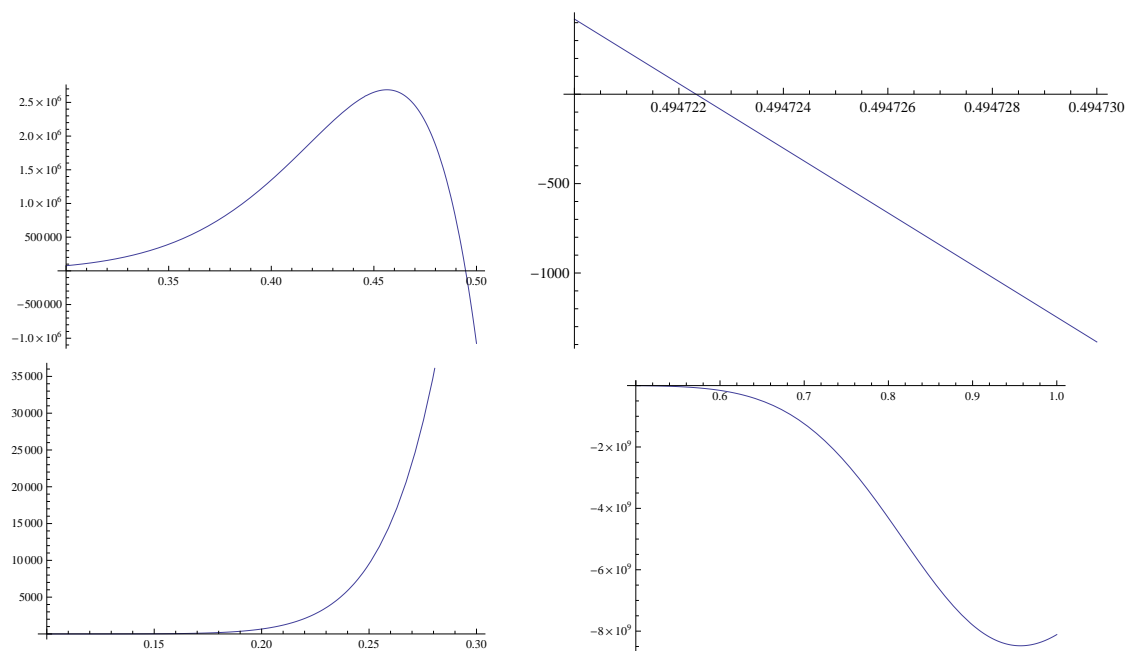

Figure 4.2: $\operatorname{det} W$ (the real axis is defined by $a)$

Also, substituting $a=0.3,0.5$ to $W_{2}-W_{1}$, we have the following sets of the eigenvalues:

$\{17.7528,17.752,11.0056,11.0005,5.33466,-1.74533,0.117449,0.0869349$, $0.0207703,0.01538,0,0,0,0,0,0,0,0\}$,

$\{14.0747,13.9953,9.92996,9.22538,9.22362,0.719009,0.359877,0.214733$, $0.100869,0.0860548,0,0,0,0,0,0,0,0\}$.

Therefore, we conclude that

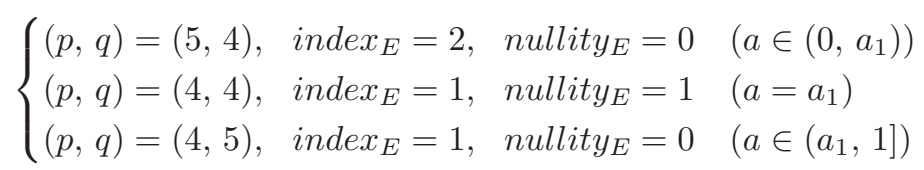

\section{3 tP family, tD family}

The curve $\operatorname{det} W$ may meet the real axis at two points $a_{1}, a_{2}$ (see Figure 4.3). Thus we consider three intervals $\left(2, a_{1}\right),\left(a_{1}, a_{2}\right),\left(a_{2}, \infty\right)$. Sets of eigenvalues of $W$ for $a=7.4028405832965,28.7783236867029$ are given by

$$
\begin{aligned}
& \{80.9577,48.893,45.7276,40.9177,-1.74202,-0.459907,-0.182299, \\
& \quad-0.124155,0\}, \\
& \{34.5772,22.6075,19.4385,18.7545,-0.858857,-0.0828255,-0.0605294, \\
& \quad-0.00120275,0\},
\end{aligned}
$$

respectively. Hence $a_{1} \approx 7.40284$ and $a_{2} \approx 28.7783$. 

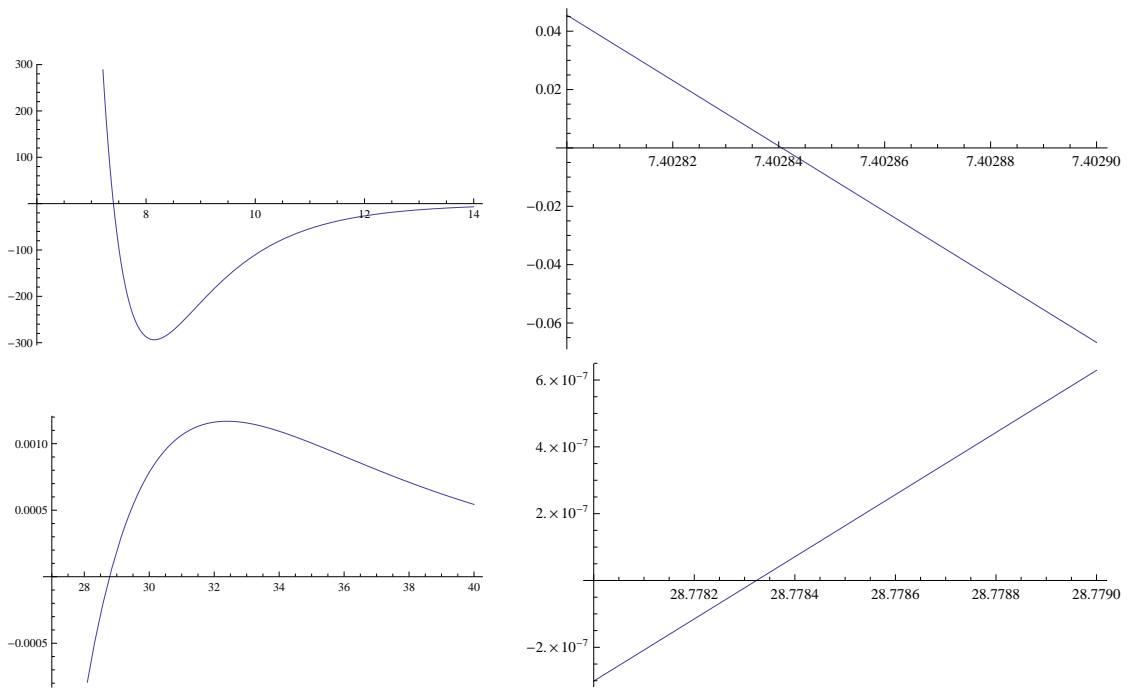

Figure 4.3: $\quad \operatorname{det} W($ the real axis is defined by $a)$

Substituting $a=7,14,30$ to $W$, we obtain sets of the eigenvalues as follows:

$$
\begin{aligned}
& \{84.0549,50.6269,47.5056,42.1118,-1.82758,-0.502372,-0.195009, \\
& \quad-0.131043,0.00417493\}, \\
& \{53.6301,33.4006,29.9484,29.3214,-1.15324,-0.175836,-0.133389, \\
& \quad-0.0334135,-0.00481829\}, \\
& \{33.7419,22.1296,18.9583,18.2612,-0.8469,-0.080107,-0.056963, \\
& \quad-0.00109614,0.000716405\} .
\end{aligned}
$$

Also, substituting $a=7,14,30$ to $W_{2}-W_{1}$, we have the following sets of the eigenvalues:

$\{6.0379,5.37072,1.57278,1.28479,1.17983,0.510969,0.329481,0.207524$,

$0.0869196,-0.00442925,0,0,0,0,0,0,0,0\}$,

$\{4.01549,3.82079,0.660116,0.450696,0.404925,0.209065,0.146808,0.0499664$,

$0.0163956,0.00721722,0,0,0,0,0,0,0,0\}$,

$\{3.06952,3.02215,0.269261,0.151882,0.13806,0.116333,0.0594526,0.00268534$, $0.00188477,-0.000794133,0,0,0,0,0,0,0,0\}$. 
Therefore, we conclude that

$$
\left\{\begin{array}{llll}
(p, q)=(5,4), & \text { index }_{E}=2, & \text { nullity }_{E}=0 & \left(a \in\left(2, a_{1}\right)\right) \\
(p, q)=(4,5), & \text { index }_{E}=1, & \text { nullity }_{E}=0 & \left(a \in\left(a_{1}, a_{2}\right)\right) \\
(p, q)=(5,4), & \text { index }_{E}=2, & \text { nullity } \\
E & =0 & \left(a \in\left(a_{2}, \infty\right)\right) \\
(p, q)=(4,4), & \text { index }_{E}=1, & \text { nullity }_{E}=1 & \left(a=a_{1}, a_{2}\right)
\end{array}\right.
$$

\section{4 tCLP family}
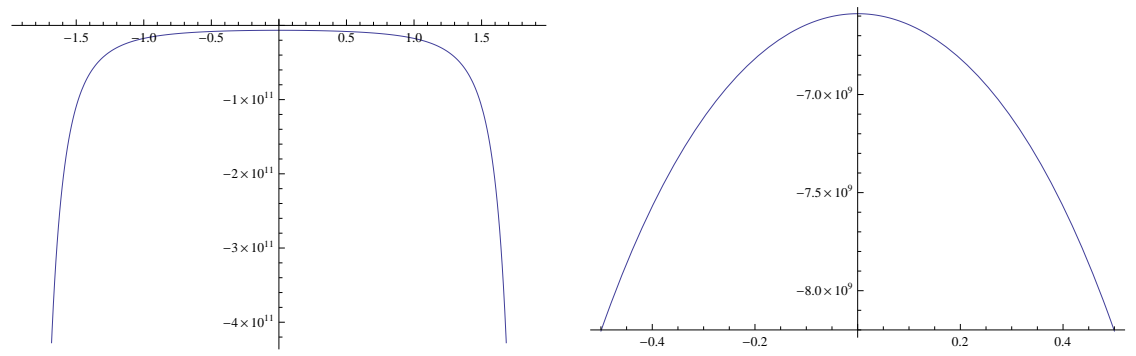

Figure 4.4: $\operatorname{det} W$ (the real axis is defined by $a)$

The curve det $W$ may not meet the real axis (see Figure 4.4). Substituting $a=0$ to $W$, we obtain a set of the eigenvalues as follows:

$$
\begin{aligned}
& \{169.074,106.977,101.864,74.8337,-7.06191,-3.56035,-3.24383, \\
& \quad 2.28679,0.25619\} .
\end{aligned}
$$

Also, substituting $a=0$ to $W_{2}-W_{1}$, we have the following set of the eigenvalues:

$$
\begin{aligned}
& \{15.791,15.791,8.75673,8.75673,7.27068,7.27068,5.06466,5.06466 \\
& \quad-0.529658,-0.529658,0,0,0,0,0,0,0,0\} .
\end{aligned}
$$

Therefore, we conclude that

$$
(p, q)=(6,3), \text { index }_{E}=3, \text { nullity }_{E}=0
$$

for $a \in(-2,2)$.

\section{Appendix (canonical homology bases and pe- riod matrices)}

We shall give a canonical homology basis and calculate periods of the abelian differentials of the second kind on each Riemann surface defined in $\S 1$. 


\section{$5.1 \quad H$ family}

\subsubsection{Canonical homology basis}

Let $M$ be a hyperelliptic Riemann surface of genus 3 defined as the completion of $\left\{(z, w) \mid w^{2}=z\left(z^{3}-a^{3}\right)\left(z^{3}-\frac{1}{a^{3}}\right)\right\} \subset \mathbb{C}^{2}$ for $a \in(0,1)$. The three differentials

$$
\frac{d z}{w}, z \frac{d z}{w}, z^{2} \frac{d z}{w}
$$

form a basis for the abelian differentials of the first kind, that is, holomorphic differentials (see. p.255 in [6]). Up to exact forms, the abelian differentials of the second kind are given by the following six differentials (see p.460 in [6]):

$$
\frac{d z}{w}, z \frac{d z}{w}, z^{2} \frac{d z}{w}, \frac{z^{4}}{w^{3}} d z, \frac{z^{5}}{w^{3}} d z, \frac{z^{6}}{w^{3}} d z .
$$

In fact, we have the following divisors:

$$
\begin{aligned}
\left(\frac{z^{4}}{w^{3}} d z\right)= & 6(0,0)-2(a, 0)-2\left(e^{\frac{2}{3} \pi i} a, 0\right)-2\left(e^{-\frac{2}{3} \pi i} a, 0\right) \\
& -2(1 / a, 0)-2\left(e^{\frac{2}{3} \pi i} / a, 0\right)-2\left(e^{-\frac{2}{3} \pi i} / a, 0\right)+10(\infty, \infty) \\
\left(\frac{z^{5}}{w^{3}} d z\right)= & 8(0,0)-2(a, 0)-2\left(e^{\frac{2}{3} \pi i} a, 0\right)-2\left(e^{-\frac{2}{3} \pi i} a, 0\right) \\
& -2(1 / a, 0)-2\left(e^{\frac{2}{3} \pi i} / a, 0\right)-2\left(e^{-\frac{2}{3} \pi i} / a, 0\right)+8(\infty, \infty) \\
\left(\frac{z^{6}}{w^{3}} d z\right)= & 10(0,0)-2(a, 0)-2\left(e^{\frac{2}{3} \pi i} a, 0\right)-2\left(e^{-\frac{2}{3} \pi i} a, 0\right) \\
& -2(1 / a, 0)-2\left(e^{\frac{2}{3} \pi i} / a, 0\right)-2\left(e^{-\frac{2}{3} \pi i} / a, 0\right)+6(\infty, \infty)
\end{aligned}
$$

Thus, the above three differentials are meromorphic. We now see that they have no residues. To do this, we only consider residues at the pole $(a, 0)$ because others can be showed in the same way. The implicit function theorem assures us that $w$ is a holomorphic coordinate around poles. We can write

$$
z=a+a_{2} w^{2}+a_{4} w^{4}+\cdots \quad\left(a_{2} \neq 0\right)
$$

around $(a, 0)$ and find

$$
\frac{z^{j}}{w^{3}} d z=\frac{\left(a+a_{2} w^{2}+a_{4} w^{4}+\cdots\right)^{j}\left(2 a_{2}+4 a_{4} w^{2}+\cdots\right)}{w^{2}} d w
$$

for $j=4,5,6$. So they have no residues at $(a, 0)$.

Next, let

$$
G=i\left(\frac{1-z^{2}}{w}, \frac{i\left(1+z^{2}\right)}{w}, \frac{2 z}{w}, \frac{z^{4}-z^{6}}{w^{3}}, \frac{i\left(z^{4}+z^{6}\right)}{w^{3}}, \frac{z^{5}}{w^{3}}\right)^{t} d z
$$

and consider the biholomorphisms

$$
j(z, w)=(z,-w), \varphi(z, w)=\left(e^{\frac{2 \pi}{3} i} z, e^{\frac{\pi}{3} i} w\right)
$$


on $M$. Then it is straightforward to compute that

$$
j^{*} G=-G, \quad \varphi^{*} G=\left(\begin{array}{cccccc}
\frac{1}{2} & \frac{\sqrt{3}}{2} & 0 & 0 & 0 & 0 \\
-\frac{\sqrt{3}}{2} & \frac{1}{2} & 0 & 0 & 0 & 0 \\
0 & 0 & -1 & 0 & 0 & 0 \\
0 & 0 & 0 & \frac{1}{2} & \frac{\sqrt{3}}{2} & 0 \\
0 & 0 & 0 & -\frac{\sqrt{3}}{2} & \frac{1}{2} & 0 \\
0 & 0 & 0 & 0 & 0 & -1
\end{array}\right) G .
$$

Now we determine a canonical homology basis on $M$. Recall that

$$
\begin{aligned}
& \pi_{\mathrm{H}}: M \longrightarrow \overline{\mathbb{C}}:=\mathbb{C} \cup\{\infty\} \\
&(z, w) \longmapsto z
\end{aligned}
$$

defines a two-sheeted branched covering having branch locus

$$
\left\{(0,0),(\infty, \infty),(a, 0),\left(e^{ \pm \frac{2}{3} \pi i} a, 0\right),(1 / a, 0),\left(e^{ \pm \frac{2}{3} \pi i} / a, 0\right)\right\}
$$

and $j$ is its deck transformation. So $M$ can be expressed as a 2-sheeted branched cover of $\overline{\mathbb{C}}$ in the following way.
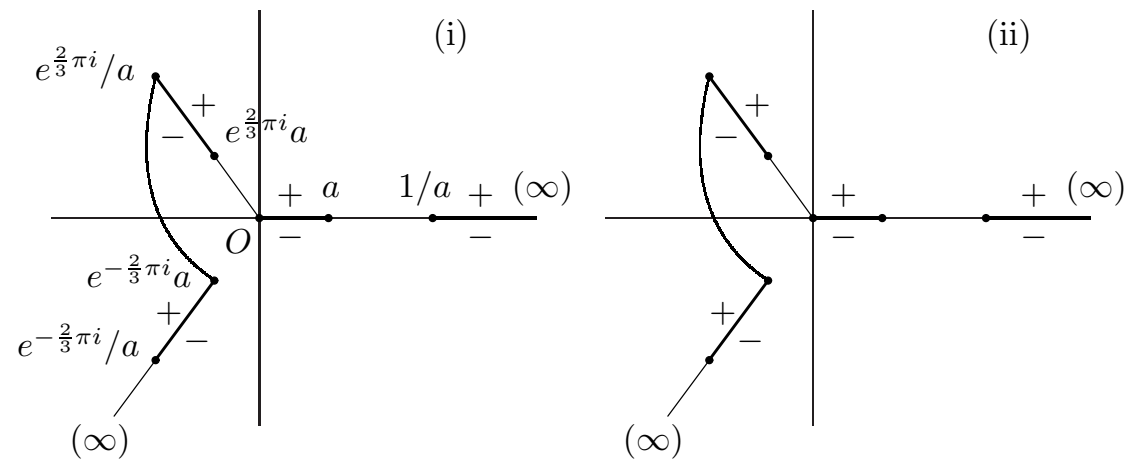

figure (H)

We prepare two copies of $\overline{\mathbb{C}}$ and slit them along the thick lines in figure $(H)$. Identifying each of the upper (resp. lower) edges of the thick lines in (i) with each of the lower (resp. upper) edges of the thick lines in (ii), we obtain the hyperelliptic Riemann surface $M$ of genus 3 (see the following figure). Note that each of thin lines joining two branch points in figure $(\mathrm{H})$ is corresponding to each of thick lines joining two branch points in the following figure. 


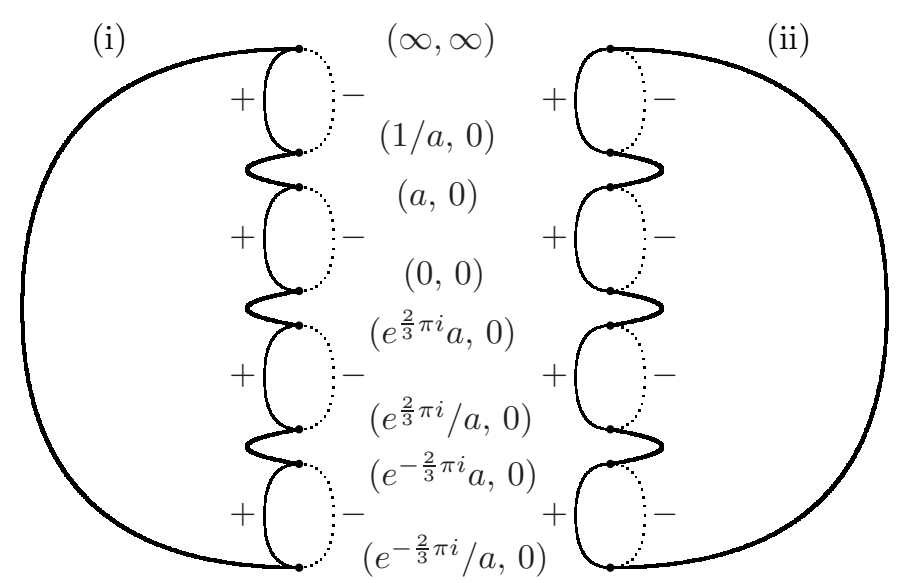

To describe 1-cycles on $M$, we consider the following key paths:

$$
\begin{aligned}
& C_{1}=\left\{(z, w)=\left(a t, a^{2} \sqrt{t\left(1-t^{3}\right)\left(1 / a^{3}-a^{3} t^{3}\right)}\right) \mid t: 0 \rightarrow 1, \sqrt{*}>0\right\}, \\
& C_{2}=\left\{(z, w)=\left(t,-i \sqrt{t\left(t^{3}-a^{3}\right)\left(1 / a^{3}-t^{3}\right)}\right) \mid t: a \rightarrow 1 / a, \sqrt{*}>0\right\} .
\end{aligned}
$$

We first choose $C_{1}$ in the following figure. After that, we shall see a relation between $C_{1}$ and $C_{2}$.
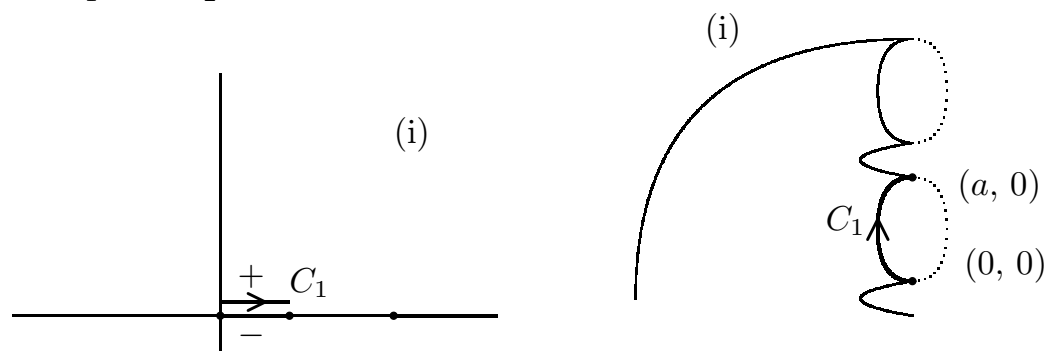

To do this, we introduce the three paths:

$$
\begin{aligned}
& C_{2}^{\prime}=\left\{(z, w)=\left(t,-i \sqrt{t\left(t^{3}-a^{3}\right)\left(1 / a^{3}-t^{3}\right)}\right) \mid t: a \rightarrow 1, \sqrt{*}>0\right\}, \\
& C_{3}=\left\{(z, w)=\left(e^{i t}, w(t)\right) \mid t: 0 \rightarrow \pi / 3, w(0) \in-i \mathbb{R}_{>0}\right\}, \\
& C_{4}=\left\{(z, w)=\left(e^{\frac{\pi}{3} i} t, e^{\frac{\pi}{6} i} \sqrt{t\left(t^{3}+a^{3}\right)\left(t^{3}+1 / a^{3}\right)}\right) \mid t: 1 \rightarrow 0, \sqrt{*}>0\right\},
\end{aligned}
$$

and claim that $C_{1} \cup\left(C_{2}^{\prime} \cup C_{3} \cup C_{4}\right)$ is homotopic to zero by using path-integrals of the holomorphic differential $\frac{1-z^{2}}{w} d z$. Note that $C_{2}^{\prime} \cap C_{3}=\{(1,0)\}$.

Straightforward calculations yield

$$
\int_{C_{1}} \frac{1-z^{2}}{w} d z=\frac{1}{a} \int_{0}^{1} \frac{1-a^{2} t^{2}}{\sqrt{t\left(1-t^{3}\right)\left(\frac{1}{a^{3}}-a^{3} t^{3}\right)}} d t
$$




$$
\begin{aligned}
& \int_{C_{2}^{\prime}} \frac{1-z^{2}}{w} d z=i \int_{a}^{1} \frac{1-t^{2}}{\sqrt{t\left(t^{3}-a^{3}\right)\left(\frac{1}{a^{3}}-t^{3}\right)}} d t \\
& \int_{C_{4}} \frac{1-z^{2}}{w} d z=-\frac{\sqrt{3}}{2} \int_{0}^{1} \frac{1+t^{2}}{\sqrt{t\left(t^{3}+a^{3}\right)\left(t^{3}+\frac{1}{a^{3}}\right)}} d t-\frac{i}{2} \int_{0}^{1} \frac{1-t^{2}}{\sqrt{t\left(t^{3}+a^{3}\right)\left(t^{3}+\frac{1}{a^{3}}\right)}} d t .
\end{aligned}
$$

Now we set $x=(z+1 / z) / 2=\cos t: 1 \rightarrow 1 / 2$ along $C_{3}$. Then $\frac{1-z^{2}}{w} d z=$ $-2 \frac{z^{2}}{w} d x . z+1 / z=2 x$ implies that $z^{2}+1 / z^{2}=4 x^{2}-2$, and thus $z^{3}+1 / z^{3}=$ $(z+1 / z)\left(z^{2}-1+1 / z^{2}\right)=8 x^{3}-6 x$. Hence,

$$
\begin{aligned}
\left(\frac{z^{2}}{w}\right)^{2} & =\frac{z^{4}}{z\left(z^{3}-a^{3}\right)\left(z^{3}-\frac{1}{a^{3}}\right)}=-\frac{1}{a^{3}+\frac{1}{a^{3}}-\left(z^{3}+\frac{1}{z^{3}}\right)} \\
& =-\frac{1}{a^{3}+\frac{1}{a^{3}}+6 x-8 x^{3}}<0 .
\end{aligned}
$$

To choose a suitable branch, we substitute $t=0$ to $z^{2} / w$. Then $\frac{z^{2}}{w}(t=0)=$ $\frac{1}{w(0)} \in i \mathbb{R}_{>0}$. As a result,

$$
\frac{z^{2}}{w}=\frac{i}{\sqrt{a^{3}+\frac{1}{a^{3}}+6 x-8 x^{3}}} \in i \mathbb{R}_{>0} .
$$

Consequently, we have

$$
\int_{C_{3}} \frac{1-z^{2}}{w} d z=2 i \int_{\frac{1}{2}}^{1} \frac{d x}{\sqrt{a^{3}+\frac{1}{a^{3}}+6 x-8 x^{3}}} .
$$

Moreover, combining (5.4) and substituting $t=\pi / 3$ to $z^{2} / w$, we find $\frac{e^{\frac{2}{3} \pi i}}{w(\pi / 3)} \in$ $i \mathbb{R}_{>0}$. So $w(\pi / 3) \in e^{\frac{\pi}{6} i} \mathbb{R}_{>0}$. Thus, $C_{3} \cap C_{4}=\left\{\left(e^{\frac{\pi}{3} i}, e^{\frac{\pi}{6} i} \sqrt{\left(1+a^{3}\right)\left(1+1 / a^{3}\right)}\right)\right\}$, and therefore, $C_{2}^{\prime} \cup C_{3} \cup C_{4}$ defines a connected path.

Since $\pi_{\mathrm{H}}\left(C_{1}\right) \cup \pi_{\mathrm{H}}\left(C_{2}^{\prime} \cup C_{3} \cup C_{4}\right)$ is homotopic to zero, there exists $n \in\{0,1\}$ such that $C_{1} \cup j^{n}\left(C_{2}^{\prime} \cup C_{3} \cup C_{4}\right)$ is homotopic to zero. It follows from (5.1), (5.2), (5.3), and (5.5) that

$$
\begin{aligned}
& \frac{1}{a} \int_{0}^{1} \frac{1-a^{2} t^{2}}{\sqrt{t\left(1-t^{3}\right)\left(\frac{1}{a^{3}}-a^{3} t^{3}\right)}} d t+(-1)^{n}\left\{i \int_{a}^{1} \frac{1-t^{2}}{\sqrt{t\left(t^{3}-a^{3}\right)\left(\frac{1}{a^{3}}-t^{3}\right)}} d t\right. \\
& -\frac{\sqrt{3}}{2} \int_{0}^{1} \frac{1+t^{2}}{\sqrt{t\left(t^{3}+a^{3}\right)\left(t^{3}+\frac{1}{a^{3}}\right)}} d t-\frac{i}{2} \int_{0}^{1} \frac{1-t^{2}}{\sqrt{t\left(t^{3}+a^{3}\right)\left(t^{3}+\frac{1}{a^{3}}\right)}} d t
\end{aligned}
$$




$$
\left.+2 i \int_{\frac{1}{2}}^{1} \frac{d x}{\sqrt{a^{3}+\frac{1}{a^{3}}+6 x-8 x^{3}}}\right\}=0 .
$$

The real part of the above implies $n=0$. Hence $C_{1} \cup\left(C_{2}^{\prime} \cup C_{3} \cup C_{4}\right)$ is homotopic to zero (see the following figure). So we obtain $C_{2}$ as follows.

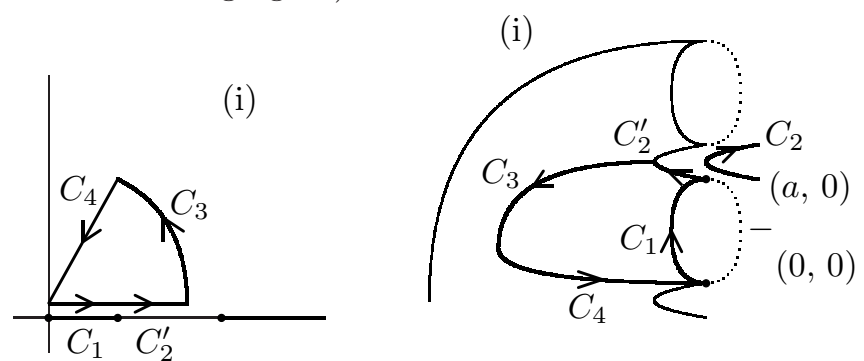

In the process, we have also showed that the two equations

$$
\begin{aligned}
& \frac{1}{a} \int_{0}^{1} \frac{1-a^{2} t^{2}}{\sqrt{t\left(1-t^{3}\right)\left(\frac{1}{a^{3}}-a^{3} t^{3}\right)}} d t=\frac{\sqrt{3}}{2} \int_{0}^{1} \frac{1+t^{2}}{\sqrt{t\left(t^{3}+a^{3}\right)\left(t^{3}+\frac{1}{a^{3}}\right)}} d t \\
& \int_{0}^{1} \frac{1-t^{2}}{\sqrt{t\left(t^{3}+a^{3}\right)\left(t^{3}+\frac{1}{a^{3}}\right)}} d t=2 \int_{a}^{1} \frac{1-t^{2}}{\sqrt{t\left(t^{3}-a^{3}\right)\left(\frac{1}{a^{3}}-t^{3}\right)}} d t+4 \int_{\frac{1}{2}}^{1} \frac{d t}{\sqrt{a^{3}+\frac{1}{a^{3}}+6 t-8 t^{3}}} .
\end{aligned}
$$

To determine other paths, we extend $C_{3}$ in the following way:

$$
C_{3}^{\prime}:=\left\{(z, w)=\left(e^{i t}, w(t)\right) \mid t: 0 \rightarrow 2 \pi / 3, w(0) \in-i \mathbb{R}_{>0}\right\} .
$$
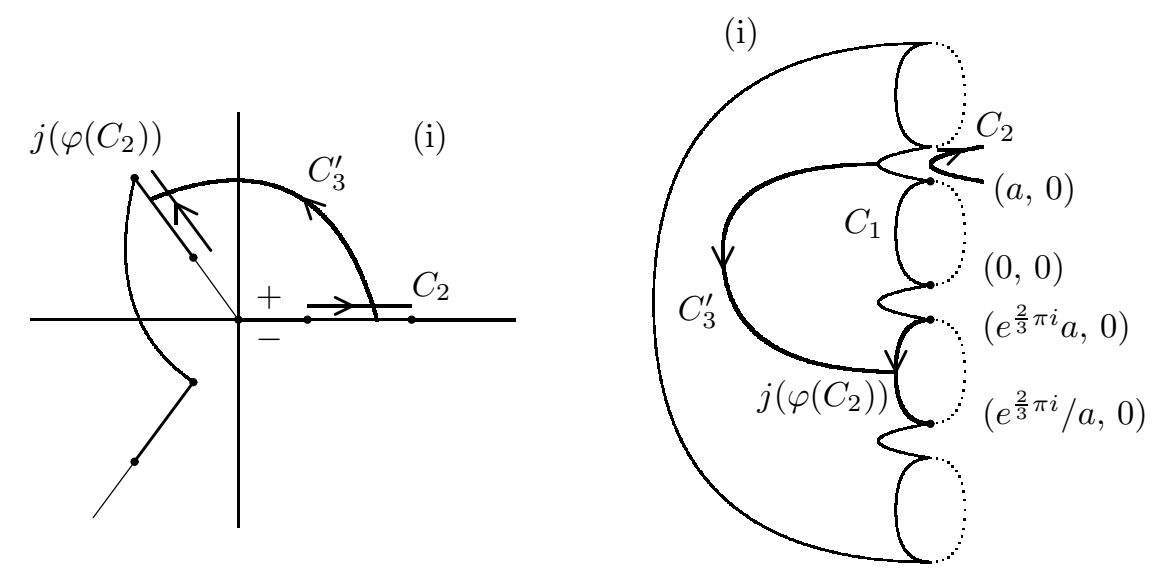

(5.4) yields $w(2 \pi / 3) \in i e^{\frac{\pi}{3} i} \mathbb{R}_{>0}$, and thus $w(2 \pi / 3)=j(\varphi(w(0)))$. It follows that $C_{3}^{\prime} \cup j\left(\varphi\left(C_{2}\right)\right)=\left\{\left(e^{\frac{2}{3} \pi i}, i e^{\frac{\pi}{3} i} \sqrt{\left(1-a^{3}\right)\left(1 / a^{3}-1\right)}\right)\right\}$. As a result, we obtain $j\left(\varphi\left(C_{2}\right)\right)$ in the previous figure. 
(i)

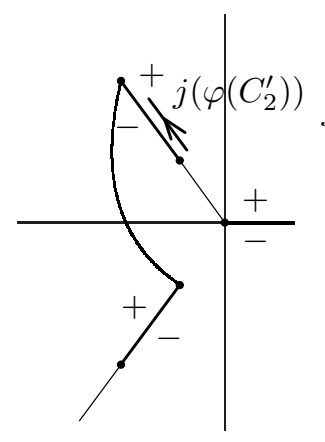

(ii)

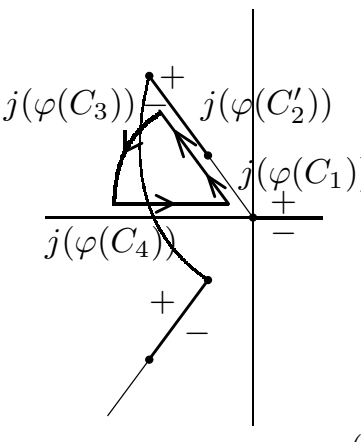

$(\infty, \infty)$

(ii)

$(1 / a, 0)$

$(a, 0)$

$(0,0)$

$\left(e^{\frac{2}{3} \pi i} a, 0\right)$

$\left(e^{\frac{2}{3} \pi i} / a, 0\right)$

$\left(e^{-\frac{2}{3} \pi i} a, 0\right)$

$\left(e^{-\frac{2}{3} \pi i} / a\right.$,
0)

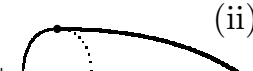<smiles>C1CCC2CCCC2C1</smiles><smiles>CC(C)(C)C1C2CCCC1(C)CCC2</smiles>

,

Note that $j\left(\varphi\left(C_{1}\right)\right) \cup j\left(\varphi\left(C_{2}^{\prime} \cup C_{3} \cup C_{4}\right)\right)$ is homotopic to zero. Since the upper edges of the thick lines in (i) is identified with the lower edges of the thick lines in (ii) in figure $(\mathrm{H}), j\left(\varphi\left(C_{1}\right)\right), j\left(\varphi\left(C_{2}^{\prime}\right)\right), j\left(\varphi\left(C_{3}\right)\right), j\left(\varphi\left(C_{4}\right)\right)$ lie in (ii) (see the above figure).

Next, $j\left(\varphi\left(C_{3}^{\prime}\right)\right)$ lies in (ii) (see the following figure). In the following left figure, $j\left(\varphi\left(C_{3}^{\prime}\right)\right)$ meets the thin line joining two branch points. Hence, $j\left(\varphi\left(C_{3}^{\prime}\right)\right)$ is given in the following right figure. Moreover, it is easy to verify that $j\left(\varphi\left(C_{3}^{\prime}\right)\right)$ meets $\varphi^{2}\left(C_{2}\right)\left(=(j \circ \varphi)\left(j\left(\varphi\left(C_{2}\right)\right)\right)\right)$ at the end in a similar way as the above.
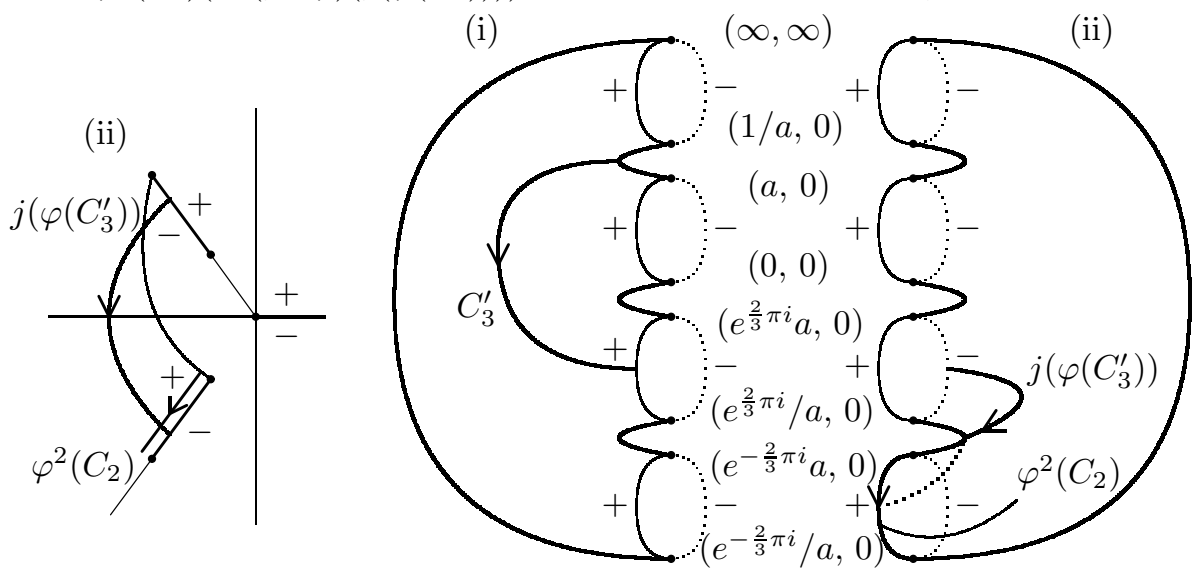

Finally, $\varphi^{2}\left(C_{1}\right), \varphi^{2}\left(C_{2}^{\prime}\right), \varphi^{2}\left(C_{3}\right), \varphi^{2}\left(C_{4}\right)$ lie in (i) and $\varphi^{2}\left(C_{1}\right) \cup \varphi^{2}\left(C_{2}^{\prime} \cup C_{3} \cup C_{4}\right)$ is homotopic to zero. So we obtain the following figure. 


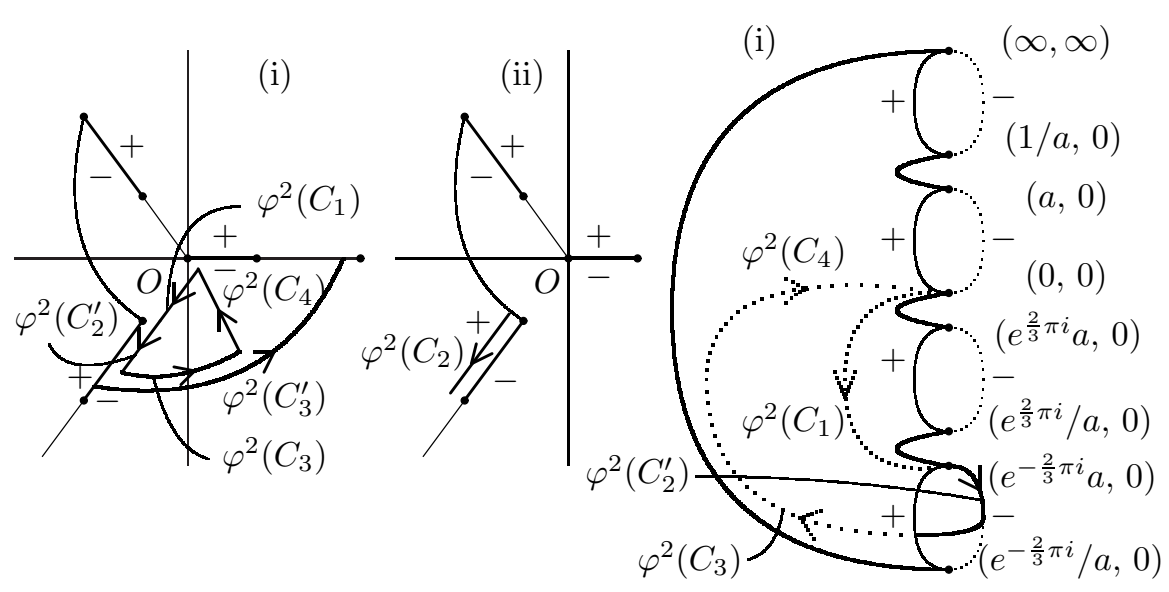

Therefore, setting

$$
\begin{aligned}
& A_{3}:=-\varphi\left(C_{2}\right)-\varphi\left(C_{1}\right)+\varphi^{2}\left(C_{1}\right)+j\left(\varphi\left(C_{2}\right)\right)+j\left(\varphi\left(C_{1}\right)\right)-j\left(\varphi^{2}\left(C_{1}\right)\right), \\
& B_{3}:=\varphi^{2}\left(C_{2}\right)-j\left(\varphi^{2}\left(C_{2}\right)\right), \quad A_{2}:=\varphi\left(C_{1}\right)-j\left(\varphi\left(C_{1}\right)\right), \quad A_{1}:=-C_{2}+j\left(C_{2}\right), \\
& B_{2}:=\varphi\left(C_{2}\right)-j\left(\varphi\left(C_{2}\right)\right)+B_{3}, B_{1}:=C_{1}-j\left(C_{1}\right)+B_{2},
\end{aligned}
$$

we obtain a canonical homology basis as follows.

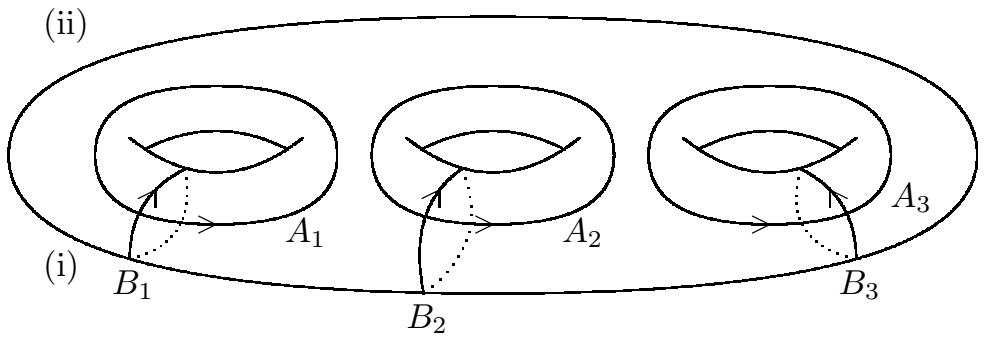

\subsubsection{Period matrix}

Key 1-cycles of the canonical homology basis as in $\S 5.1 .1$ are given by $C_{1} \cup$ $\left\{-j\left(C_{1}\right)\right\}$ and $C_{2} \cup\left\{-j\left(C_{2}\right)\right\}$. Since the two key 1-cycles meet the pole $(a, 0)$ of the $z^{j} / w^{3} d z$ 's, we introduce two useful 1-cycles. First we extend $C_{3}$ in the following way.
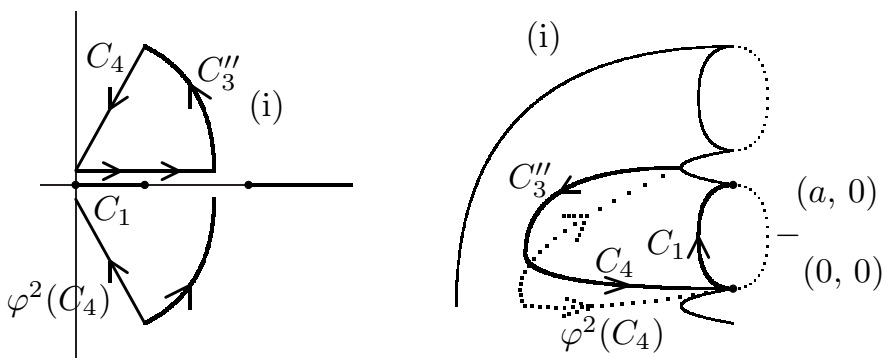


$$
C_{3}^{\prime \prime}:=\left\{(z, w)=\left(e^{i t}, w(t)\right) \mid t:-\pi / 3 \rightarrow \pi / 3, w(0) \in-i \mathbb{R}_{>0}\right\} .
$$

By the arguments as in $\S$ 5.1.1 we conclude that $C_{1} \cup\left\{-j\left(C_{1}\right)\right\}$ is homotopic to $\left(-C_{4}\right) \cup\left(-C_{3}^{\prime \prime}\right) \cup \varphi^{2}\left(C_{4}\right)$. Next we introduce the following extension of $C_{4}$.

$$
C_{4}^{\prime}=\left\{(z, w)=\left(e^{\frac{\pi}{3} i} t, e^{\frac{\pi}{6} i} \sqrt{t\left(t^{3}+a^{3}\right)\left(t^{3}+1 / a^{3}\right)}\right) \mid t: \infty \rightarrow 0, \sqrt{*}>0\right\} .
$$
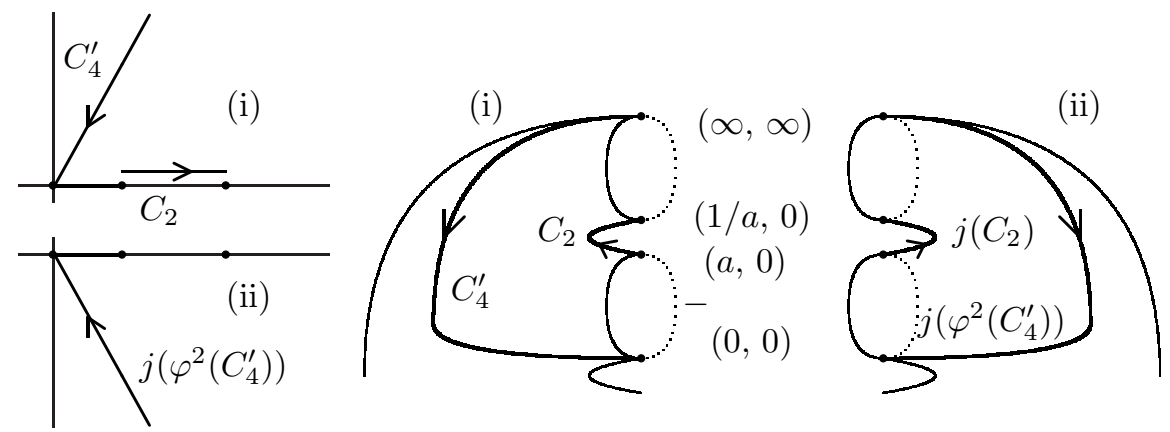

The previous arguments as in $\S 5.1 .1$ imply that $C_{2} \cup\left\{-j\left(C_{2}\right)\right\}$ is homotopic to $\left(-C_{4}^{\prime}\right) \cup j\left(\varphi^{2}\left(C_{4}^{\prime}\right)\right)$. Hence we may consider $\left(-C_{4}\right) \cup\left(-C_{3}^{\prime \prime}\right) \cup \varphi^{2}\left(C_{4}\right)$ and $\left(-C_{4}^{\prime}\right) \cup j\left(\varphi^{2}\left(C_{4}^{\prime}\right)\right)$ instead of $C_{1} \cup\left\{-j\left(C_{1}\right)\right\}$ and $C_{2} \cup\left\{-j\left(C_{2}\right)\right\}$, respectively.

Setting $x=(z+1 / z) / 2=\cos t: 1 / 2 \rightarrow 1 \rightarrow 1 / 2$ along $C_{3}^{\prime \prime}$, we find

$$
\frac{z^{2}-1}{2 i z}=\sin t= \begin{cases}-\sqrt{1-x^{2}} & \left(t:-\frac{\pi}{3} \rightarrow 0\right) \\ \sqrt{1-x^{2}} & \left(t: 0 \rightarrow \frac{\pi}{3}\right)\end{cases}
$$

It follows from (5.4) that

$$
\begin{aligned}
& \int_{C_{3}^{\prime \prime}} \frac{1-z^{2}}{w} d z=-2 i \int_{\frac{1}{2}}^{1} \frac{d x}{\sqrt{a^{3}+\frac{1}{a^{3}}+6 x-8 x^{3}}}-2 i \int_{1}^{\frac{1}{2}} \frac{d x}{\sqrt{a^{3}+\frac{1}{a^{3}}+6 x-8 x^{3}}}=0, \\
& \int_{C_{3}^{\prime \prime}} \frac{i\left(1+z^{2}\right)}{w} d z=-2 i \int_{\frac{1}{2}}^{1} \frac{x}{\sqrt{\left(a^{3}+\frac{1}{a^{3}}+6 x-8 x^{3}\right)\left(1-x^{2}\right)}} d x \\
& +2 i \int_{1}^{\frac{1}{2}} \frac{x}{\sqrt{\left(a^{3}+\frac{1}{a^{3}}+6 x-8 x^{3}\right)\left(1-x^{2}\right)}} d x=-4 i \int_{\frac{1}{2}}^{1} \frac{x}{\sqrt{\left(a^{3}+\frac{1}{a^{3}}+6 x-8 x^{3}\right)\left(1-x^{2}\right)}} d x \\
& \int_{C_{3}^{\prime \prime}} \frac{2 z}{w} d z=-4 \int_{\frac{1}{2}}^{1} \frac{x}{\sqrt{\left(a^{3}+\frac{1}{a^{3}}+6 x-8 x^{3}\right)\left(1-x^{2}\right)}} \\
& \int_{C_{3}^{\prime \prime}} \frac{z^{4}-z^{6}}{w^{3}} d z=0, \\
& \int_{C_{3}^{\prime \prime}} \frac{i\left(z^{4}+z^{6}\right)}{w^{3}} d z=4 i \int_{\frac{1}{2}}^{1} \frac{x}{\sqrt{a^{3}+\frac{1}{a^{3}}+6 x-8 x^{3}} \sqrt{1-x^{2}}} d x
\end{aligned}
$$




$$
\int_{C_{3}^{\prime \prime}} \frac{z^{5}}{w^{3}} d z=2 \int_{\frac{1}{2}}^{1} \frac{d x}{{\sqrt{a^{3}+\frac{1}{a^{3}}+6 x-8 x^{3}}}^{3} \sqrt{1-x^{2}}} .
$$

Straightforward calculation yields

$$
\begin{aligned}
& \int_{C_{4}} \frac{i\left(1+z^{2}\right)}{w} d z=\frac{1}{2} \int_{0}^{1} \frac{1+t^{2}}{\sqrt{t\left(t^{3}+a^{3}\right)\left(t^{3}+\frac{1}{a^{3}}\right)}} d t-\frac{\sqrt{3}}{2} i \int_{0}^{1} \frac{1-t^{2}}{\sqrt{t\left(t^{3}+a^{3}\right)\left(t^{3}+\frac{1}{a^{3}}\right)}} d t, \\
& \int_{C_{4}} \frac{2 z}{w} d z=-2 i \int_{0}^{1} \frac{t}{\sqrt{t\left(t^{3}+a^{3}\right)\left(t^{3}+\frac{1}{a^{3}}\right)}} d t \\
& \int_{C_{4}} \frac{z^{4}-z^{6}}{w^{3}} d z=\frac{i}{2} \int_{0}^{1} \frac{t^{4}-t^{6}}{{\sqrt{t\left(t^{3}+a^{3}\right)\left(t^{3}+\frac{1}{a^{3}}\right)}}^{3}} d t+\frac{\sqrt{3}}{2} \int_{0}^{1} \frac{t^{4}+t^{6}}{{\sqrt{t\left(t^{3}+a^{3}\right)\left(t^{3}+\frac{1}{a^{3}}\right)}}^{3}} d t, \\
& \int_{C_{4}} \frac{i\left(z^{4}+z^{6}\right)}{w^{3}} d z=\frac{\sqrt{3}}{2} i \int_{0}^{1} \frac{t^{4}-t^{6}}{{\sqrt{t\left(t^{3}+a^{3}\right)\left(t^{3}+{\frac{1}{a^{3}}}^{3}\right.}}^{3}} d t-\frac{1}{2} \int_{0}^{1} \frac{t^{4}+t^{6}}{{\sqrt{t\left(t^{3}+a^{3}\right)\left(t^{3}+\frac{1}{a^{3}}\right)}}^{3}} d t, \\
& \int_{C_{4}} \frac{z^{5}}{w^{3}} d z=i \int_{0}^{1} \frac{t^{5}}{{\sqrt{t\left(t^{3}+a^{3}\right)\left(t^{3}+\frac{1}{a^{3}}\right)}}^{3}} d t
\end{aligned}
$$

Combining these equations and (5.3), we have

$$
\begin{aligned}
& \int_{\left(-C_{4}\right) \cup\left(-C_{3}^{\prime \prime}\right) \cup \varphi^{2}\left(C_{4}\right)} \frac{1-z^{2}}{w} d z=\sqrt{3} \int_{0}^{1} \frac{1+t^{2}}{\sqrt{t\left(t^{3}+a^{3}\right)\left(t^{3}+\frac{1}{a^{3}}\right)}} d t, \\
& \int_{\left(-C_{4}\right) \cup\left(-C_{3}^{\prime \prime}\right) \cup \varphi^{2}\left(C_{4}\right)} \frac{i\left(1+z^{2}\right)}{w} d z=\sqrt{3} i \int_{0}^{1} \frac{1-t^{2}}{\sqrt{t\left(t^{3}+a^{3}\right)\left(t^{3}+\frac{1}{a^{3}}\right)}} d t \\
& +4 i \int_{\frac{1}{2}}^{1} \frac{x}{\sqrt{\left(a^{3}+\frac{1}{a^{3}}+6 x-8 x^{3}\right)\left(1-x^{2}\right)}} d x \text {, } \\
& \int_{\left(-C_{4}\right) \cup\left(-C_{3}^{\prime \prime}\right) \cup \varphi^{2}\left(C_{4}\right)} \frac{2 z}{w} d z=4 \int_{\frac{1}{2}}^{1} \frac{d x}{\sqrt{\left(a^{3}+\frac{1}{a^{3}}+6 x-8 x^{3}\right)\left(1-x^{2}\right)}}, \\
& \int_{\left(-C_{4}\right) \cup\left(-C_{3}^{\prime \prime}\right) \cup \varphi^{2}\left(C_{4}\right)} \frac{z^{4}-z^{6}}{w^{3}} d z=-\sqrt{3} \int_{0}^{1} \frac{t^{4}+t^{6}}{{\sqrt{t\left(t^{3}+a^{3}\right)\left(t^{3}+\frac{1}{a^{3}}\right)}}^{3}} d t, \\
& \int_{\left(-C_{4}\right) \cup\left(-C_{3}^{\prime \prime}\right) \cup \varphi^{2}\left(C_{4}\right)} \frac{i\left(z^{4}+z^{6}\right)}{w^{3}} d z=-\sqrt{3} i \int_{0}^{1} \frac{t^{4}-t^{6}}{{\sqrt{t\left(t^{3}+a^{3}\right)\left(t^{3}+\frac{1}{a^{3}}\right)}}^{3}} d t
\end{aligned}
$$




$$
\begin{gathered}
-4 i \int_{\frac{1}{2}}^{1} \frac{x}{{\sqrt{a^{3}+\frac{1}{a^{3}}+6 x-8 x^{3}}}^{3}{\sqrt{1-x^{2}}}^{1}} d x, \\
\int_{\left(-C_{4}\right) \cup\left(-C_{3}^{\prime \prime}\right) \cup \varphi^{2}\left(C_{4}\right)} \frac{z^{5}}{w^{3}} d z=-2 \int_{\frac{1}{2}}^{1} \frac{d x}{{\sqrt{a^{3}+\frac{1}{a^{3}}+6 x-8 x^{3}} \sqrt{1-x^{2}}}^{3}}
\end{gathered}
$$

Similarly we obtain

$$
\begin{aligned}
& \int_{C_{4}^{\prime}} \frac{1-z^{2}}{w} d z=-\frac{\sqrt{3}}{2} \int_{0}^{\infty} \frac{1+t^{2}}{\sqrt{t\left(t^{3}+a^{3}\right)\left(t^{3}+\frac{1}{a^{3}}\right)}} d t-\frac{i}{2} \int_{0}^{\infty} \frac{1-t^{2}}{\sqrt{t\left(t^{3}+a^{3}\right)\left(t^{3}+\frac{1}{a^{3}}\right)}} d t \\
& =-\sqrt{3} \int_{0}^{1} \frac{1+t^{2}}{\sqrt{t\left(t^{3}+a^{3}\right)\left(t^{3}+\frac{1}{a^{3}}\right)}} d t \\
& \int_{C_{4}^{\prime}} \frac{i\left(1+z^{2}\right)}{w} d z=\int_{0}^{1} \frac{1+t^{2}}{\sqrt{t\left(t^{3}+a^{3}\right)\left(t^{3}+\frac{1}{a^{3}}\right)}} d t, \\
& \int_{C_{4}^{\prime}} \frac{2 z}{w} d z=-4 i \int_{0}^{1} \frac{t}{\sqrt{t\left(t^{3}+a^{3}\right)\left(t^{3}+\frac{1}{a^{3}}\right)}} d t \\
& \int_{C_{4}^{\prime}} \frac{z^{4}-z^{6}}{w^{3}} d z=\sqrt{3} \int_{0}^{1} \frac{t^{4}+t^{6}}{{\sqrt{t\left(t^{3}+a^{3}\right)\left(t^{3}+{\frac{1}{a^{3}}}^{3}\right.}}^{3}} d t \\
& \int_{C_{4}^{\prime}} \frac{i\left(z^{4}+z^{6}\right)}{w^{3}} d z=-\int_{0}^{1} \frac{t^{4}+t^{6}}{{\sqrt{t\left(t^{3}+a^{3}\right)\left(t^{3}+\frac{1}{a^{3}}\right)}}^{3}} d t \\
& \int_{C_{4}^{\prime}} \frac{z^{5}}{w^{3}} d z=2 i \int_{0}^{1} \frac{t^{5}}{{\sqrt{t\left(t^{3}+a^{3}\right)\left(t^{3}+\frac{1}{a^{3}}\right)}}^{3}} d t .
\end{aligned}
$$

Hence we find

$$
\begin{aligned}
\int_{\left(-C_{4}^{\prime}\right) \cup j\left(\varphi^{2}\left(C_{4}^{\prime}\right)\right)} \frac{1-z^{2}}{w} d z & =0 \\
\int_{\left(-C_{4}^{\prime}\right) \cup j\left(\varphi^{2}\left(C_{4}^{\prime}\right)\right)} \frac{i\left(1+z^{2}\right)}{w} d z & =-2 \int_{0}^{1} \frac{1+t^{2}}{\sqrt{t\left(t^{3}+a^{3}\right)\left(t^{3}+\frac{1}{a^{3}}\right)}} d t \\
\int_{\left(-C_{4}^{\prime}\right) \cup j\left(\varphi^{2}\left(C_{4}^{\prime}\right)\right)} \frac{2 z}{w} d z & =8 i \int_{0}^{1} \frac{t}{\sqrt{t\left(t^{3}+a^{3}\right)\left(t^{3}+\frac{1}{a^{3}}\right)}} d t \\
\int_{\left(-C_{4}^{\prime}\right) \cup j\left(\varphi^{2}\left(C_{4}^{\prime}\right)\right)} \frac{z^{4}-z^{6}}{w^{3}} d z & =0, \\
\int_{\left(-C_{4}^{\prime}\right) \cup j\left(\varphi^{2}\left(C_{4}^{\prime}\right)\right)} \frac{i\left(z^{4}+z^{6}\right)}{w^{3}} d z & =2 \int_{0}^{1} \frac{t^{4}+t^{6}}{{\sqrt{t\left(t^{3}+a^{3}\right)\left(t^{3}+\frac{1}{a^{3}}\right)}}^{3}} d t
\end{aligned}
$$




$$
\int_{\left(-C_{4}^{\prime}\right) \cup j\left(\varphi^{2}\left(C_{4}^{\prime}\right)\right)} \frac{z^{5}}{w^{3}} d z=-4 i \int_{0}^{1} \frac{t^{5}}{{\sqrt{t\left(t^{3}+a^{3}\right)\left(t^{3}+\frac{1}{a^{3}}\right)}}^{3}} d t .
$$

Set

$$
\begin{aligned}
& A:=\int_{0}^{1} \frac{1+t^{2}}{\sqrt{t\left(t^{3}+a^{3}\right)\left(t^{3}+\frac{1}{a^{3}}\right)}} d t \\
& B:=\sqrt{3} \int_{0}^{1} \frac{1-t^{2}}{\sqrt{t\left(t^{3}+a^{3}\right)\left(t^{3}+\frac{1}{a^{3}}\right)}} d t+4 \int_{\frac{1}{2}}^{1} \frac{x}{\sqrt{\left(a^{3}+\frac{1}{a^{3}}+6 x-8 x^{3}\right)\left(1-x^{2}\right)}} d x, \\
& C:=4 \int_{\frac{1}{2}}^{1} \frac{d x}{\sqrt{\left(a^{3}+\frac{1}{a^{3}}+6 x-8 x^{3}\right)\left(1-x^{2}\right)}} \text {, } \\
& D:=8 \int_{0}^{1} \frac{t}{\sqrt{t\left(t^{3}+a^{3}\right)\left(t^{3}+\frac{1}{a^{3}}\right)}} d t, \quad E:=\int_{0}^{1} \frac{t^{4}+t^{6}}{{\sqrt{t\left(t^{3}+a^{3}\right)\left(t^{3}+{\frac{1}{a^{3}}}^{3}\right.}}^{3}} d t, \\
& F:=\sqrt{3} \int_{0}^{1} \frac{t^{4}-t^{6}}{{\sqrt{t\left(t^{3}+a^{3}\right)\left(t^{3}+\frac{1}{a^{3}}\right)}}^{3}} d t+4 \int_{\frac{1}{2}}^{1} \frac{x}{{\sqrt{a^{3}+{\frac{1}{a^{3}}}^{2} 6 x-8 x^{3}}}^{3}{\sqrt{1-x^{2}}}^{2}} d x, \\
& H:=2 \int_{\frac{1}{2}}^{1} \frac{d x}{{\sqrt{a^{3}+\frac{1}{a^{3}}+6 x-8 x^{3}}}^{3} \sqrt{1-x^{2}}}, \quad I:=4 \int_{0}^{1} \frac{t^{5}}{{\sqrt{t\left(t^{3}+a^{3}\right)\left(t^{3}+{\frac{1}{a^{3}}}^{3}\right.}}^{3}} d t .
\end{aligned}
$$

Using (5.6)-(5.17), we have

$$
\int_{\left(-C_{4}\right) \cup\left(-C_{3}^{\prime \prime}\right) \cup \varphi^{2}\left(C_{4}\right)} G=i\left(\begin{array}{c}
\sqrt{3} A \\
i B \\
C \\
-\sqrt{3} E \\
-i F \\
-H
\end{array}\right), \quad \int_{\left(-C_{4}^{\prime}\right) \cup j\left(\varphi^{2}\left(C_{4}^{\prime}\right)\right)} G=i\left(\begin{array}{c}
0 \\
-2 A \\
i D \\
0 \\
2 E \\
-i I
\end{array}\right) .
$$

Therefore, the period matrix of the abelian differentials of the second kind, that is, $\left(\int_{A_{1}} G \int_{A_{2}} G \int_{A_{3}} G \int_{B_{1}} G \int_{B_{2}} G \int_{B_{3}} G\right)$ is given by

$$
i\left(\begin{array}{cccccc}
0 & \frac{\sqrt{3}}{2}(A+i B) & 0 & -\sqrt{3} A & -2 \sqrt{3} A & -\sqrt{3} A \\
2 A & \frac{-3 A+i B}{2} & A-i B & i B & 0 & A \\
-i D & -C & 2 C+i D & C & 0 & i D \\
0 & -\frac{\sqrt{3}}{2}(E+i F) & 0 & \sqrt{3} E & 2 \sqrt{3} E & \sqrt{3} E \\
-2 E & \frac{3 E-i F}{2} & -E+i F & -i F & 0 & -E \\
i I & H & -2 H-i I & -H & 0 & -i I
\end{array}\right) .
$$


The arguments in next subsections are based on the above techniques, so we omit some details.

\section{2 rPD family}

\subsubsection{Canonical homology basis}

Let $M$ be a hyperelliptic Riemann surface of genus 3 defined as the completion of $\left\{(z, w) \mid w^{2}=z\left(z^{3}-a^{3}\right)\left(z^{3}+\frac{1}{a^{3}}\right)\right\} \subset \mathbb{C}^{2}$ for $a \in(0,1]$. The three differentials

$$
\frac{d z}{w}, z \frac{d z}{w}, z^{2} \frac{d z}{w}
$$

form a basis for the abelian differentials of the first kind. Up to exact forms, the abelian differentials of the second kind are given by the following six differentials:

$$
\frac{d z}{w}, z \frac{d z}{w}, z^{2} \frac{d z}{w}, \frac{z^{4}}{w^{3}} d z, \frac{z^{5}}{w^{3}} d z, \frac{z^{6}}{w^{3}} d z .
$$

Let

$$
G=i\left(\frac{1-z^{2}}{w}, \frac{i\left(1+z^{2}\right)}{w}, \frac{2 z}{w}, \frac{z^{4}-z^{6}}{w^{3}}, \frac{i\left(z^{4}+z^{6}\right)}{w^{3}}, \frac{z^{5}}{w^{3}}\right)^{t} d z
$$

and consider the biholomorphisms

$$
j(z, w)=(z,-w), \varphi(z, w)=\left(e^{\frac{2 \pi}{3} i} z, e^{\frac{\pi}{3} i} w\right)
$$

on $M$. Then it is straightforward to compute that

$$
j^{*} G=-G, \quad \varphi^{*} G=\left(\begin{array}{cccccc}
\frac{1}{2} & \frac{\sqrt{3}}{2} & 0 & 0 & 0 & 0 \\
-\frac{\sqrt{3}}{2} & \frac{1}{2} & 0 & 0 & 0 & 0 \\
0 & 0 & -1 & 0 & 0 & 0 \\
0 & 0 & 0 & \frac{1}{2} & \frac{\sqrt{3}}{2} & 0 \\
0 & 0 & 0 & -\frac{\sqrt{3}}{2} & \frac{1}{2} & 0 \\
0 & 0 & 0 & 0 & 0 & -1
\end{array}\right) G .
$$

Now we determine a canonical homology basis on $M$. Recall that

$$
\begin{gathered}
\pi_{\mathrm{rPD}}: \quad M \longrightarrow \overline{\mathbb{C}}:=\mathbb{C} \cup\{\infty\} \\
(z, w) \longmapsto z
\end{gathered}
$$

defines a two-sheeted branched covering having branch locus

$$
\left\{(0,0),(\infty, \infty),(a, 0),\left(e^{ \pm \frac{2}{3} \pi i} a, 0\right),(-1 / a, 0),\left(e^{ \pm \frac{\pi}{3} i} / a, 0\right)\right\}
$$


and $j$ is its deck transformation. So $M$ can be expressed as a 2-sheeted branched cover of $\overline{\mathbb{C}}$ in the following way.
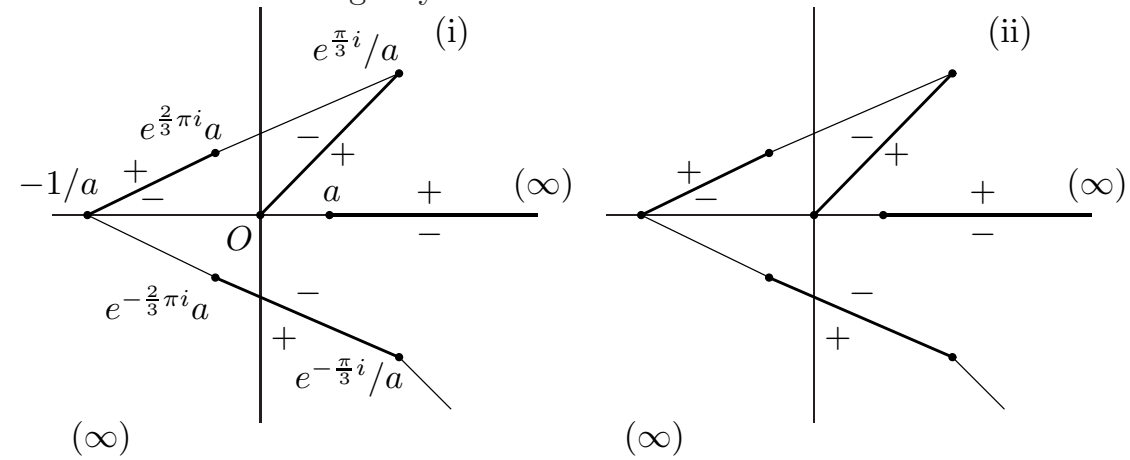

figure (rPD)

We prepare two copies of $\overline{\mathbb{C}}$ and slit them along the thick lines in figure (rPD). Identifying each of the upper (resp. lower) edges of the thick lines in (i) with each of the lower (resp. upper) edges of the thick lines in (ii), we obtain the hyperelliptic Riemann surface $M$ of genus 3 (see the following figure). Note that each of thin lines joining two branch points in figure (rPD) is corresponding to each of thick lines joining two branch points in the following figure.

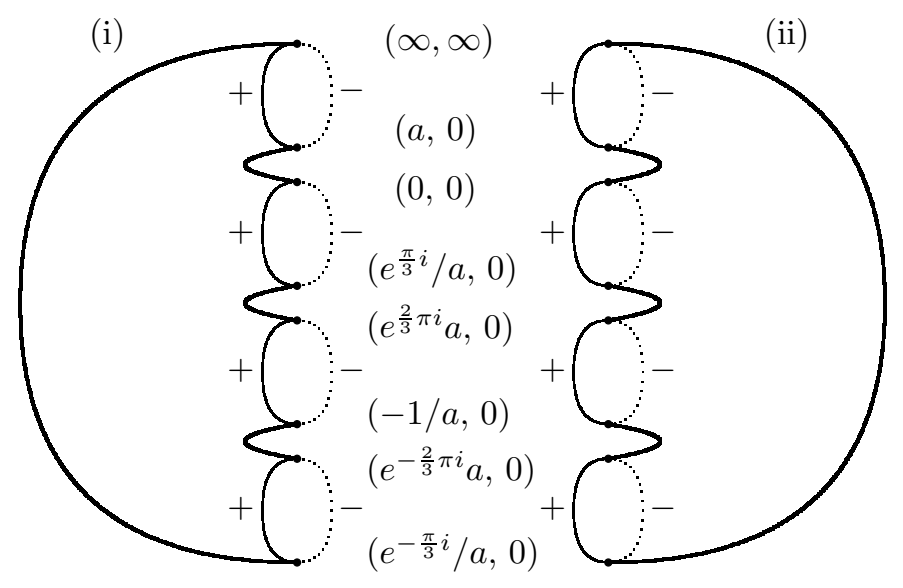

To describe 1-cycles on $M$, we consider the following key paths:

$C_{1}=\left\{(z, w)=\left(e^{\frac{\pi}{3} i} t / a, i e^{\frac{\pi}{6} i} \sqrt{t\left(1-t^{3}\right)\left(a^{3}+t^{3} / a^{3}\right)} / a^{2}\right) \mid t: 0 \rightarrow 1, \sqrt{*}>0\right\}$,
$C_{2}=\left\{(z, w)=\left(e^{\frac{\pi}{3} i} t / a,-e^{\frac{\pi}{6} i} \sqrt{t\left(t^{3}-1\right)\left(a^{3}+t^{3} / a^{3}\right)} / a^{2}\right) \mid t: 1 \rightarrow \infty, \sqrt{*}>0\right\}$,
$C_{3}=\left\{(z, w)=\left(a t, i a^{2} \sqrt{t\left(1-t^{3}\right)\left(a^{3} t^{3}+1 / a^{3}\right)}\right) \mid t: 0 \rightarrow 1, \sqrt{*}>0\right\}$,
$C_{4}=\left\{(z, w)=\left(a t, a^{2} \sqrt{t\left(t^{3}-1\right)\left(a^{3} t^{3}+1 / a^{3}\right)}\right) \mid t: 1 \rightarrow \infty, \sqrt{*}>0\right\}$.

We first choose $C_{1}$ in the following figure. After that, we shall see that $C_{1} \cup C_{2}$ 
is homotopic to $C_{3} \cup C_{4}$ by using path-integrals of the holomorphic differentials $\frac{2 z}{w} d z$ and $\frac{1-z^{2}}{w} d z$.
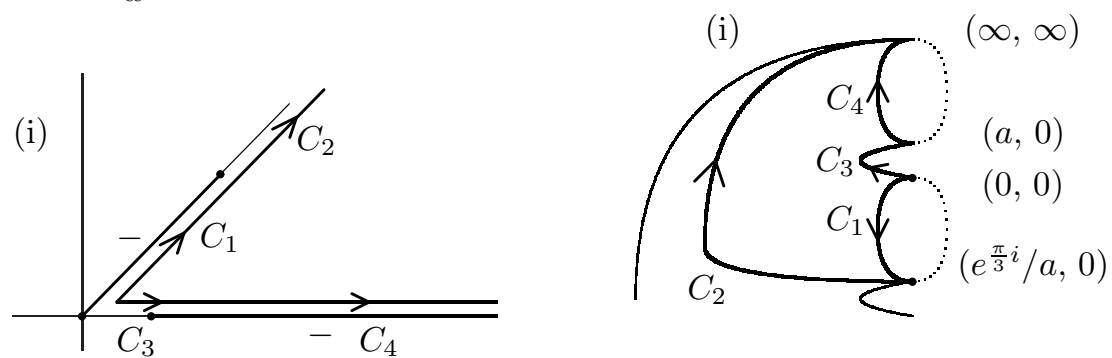

Taking suitable $l, m, n \in\{0,1\}$, we find that $C_{1} \cup j^{l}\left(C_{2}\right)$ is homotopic to $j^{m}\left(C_{3}\right) \cup j^{n}\left(C_{4}\right)$. Now we have

$$
\begin{aligned}
& \int_{C_{1}} \frac{2 z}{w} d z=2 \int_{0}^{1} \frac{t}{\sqrt{t\left(1-t^{3}\right)\left(a^{3}+\frac{t^{3}}{a^{3}}\right)}} d t=2 \int_{1}^{\infty} \frac{t}{\sqrt{t\left(t^{3}-1\right)\left(a^{3} t^{3}+\frac{1}{a^{3}}\right)}} d t \\
& \int_{C_{2}} \frac{2 z}{w} d z=-2 i \int_{0}^{1} \frac{t}{\sqrt{t\left(1-t^{3}\right)\left(a^{3} t^{3}+\frac{1}{a^{3}}\right)}} d t \\
& \int_{C_{3}} \frac{2 z}{w} d z=-2 i \int_{0}^{1} \frac{t}{\sqrt{t\left(1-t^{3}\right)\left(a^{3} t^{3}+\frac{1}{a^{3}}\right)}} d t \\
& \int_{C_{4}} \frac{2 z}{w} d z=2 \int_{1}^{\infty} \frac{t}{\sqrt{t\left(t^{3}-1\right)\left(a^{3} t^{3}+\frac{1}{a^{3}}\right)}} d t .
\end{aligned}
$$

It follows from the equation $\int_{C_{1} \cup j^{l}\left(C_{2}\right)} \frac{2 z}{w} d z=\int_{j^{m}\left(C_{3}\right) \cup j^{n}\left(C_{4}\right)} \frac{2 z}{w} d z$ that $n=0$ and $l=m$. Similarly, we find

$$
\begin{aligned}
& \int_{C_{1}} \frac{1-z^{2}}{w} d z=-\frac{\sqrt{3}}{2 a} i \int_{1}^{\infty} \frac{1+a^{2} t^{2}}{\sqrt{t\left(t^{3}-1\right)\left(a^{3} t^{3}+\frac{1}{a^{3}}\right)}} d t-\frac{1}{2 a} \int_{1}^{\infty} \frac{1-a^{2} t^{2}}{\sqrt{t\left(t^{3}-1\right)\left(a^{3} t^{3}+\frac{1}{a^{3}}\right)}} d t \\
& \int_{C_{2}} \frac{1-z^{2}}{w} d z=-\frac{\sqrt{3}}{2 a} \int_{0}^{1} \frac{1+a^{2} t^{2}}{\sqrt{t\left(1-t^{3}\right)\left(a^{3} t^{3}+\frac{1}{a^{3}}\right)}} d t+\frac{i}{2 a} \int_{0}^{1} \frac{1-a^{2} t^{2}}{\sqrt{t\left(1-t^{3}\right)\left(a^{3} t^{3}+\frac{1}{a^{3}}\right)}} d t \\
& \int_{C_{3}} \frac{1-z^{2}}{w} d z=-\frac{i}{a} \int_{0}^{1} \frac{1-a^{2} t^{2}}{\sqrt{t\left(1-t^{3}\right)\left(a^{3} t^{3}+\frac{1}{a^{3}}\right)}} d t \\
& \int_{C_{4}} \frac{1-z^{2}}{w} d z=\frac{1}{a} \int_{1}^{\infty} \frac{1-a^{2} t^{2}}{\sqrt{t\left(t^{3}-1\right)\left(a^{3} t^{3}+\frac{1}{a^{3}}\right)}} d t .
\end{aligned}
$$

The equation $\int_{C_{1} \cup j^{m}\left(C_{2}\right)} \frac{1-z^{2}}{w} d z=\int_{j^{m}\left(C_{3}\right) \cup C_{4}} \frac{1-z^{2}}{w} d z$ implies $m=0$. Thus, 
$C_{1} \cup C_{2}$ is homotopic to $C_{3} \cup C_{4}$. In the process, we have also showed that the two equations

$$
\begin{aligned}
& \sqrt{3} \int_{0}^{1} \frac{1-a^{2} t^{2}}{\sqrt{t\left(1-t^{3}\right)\left(a^{3} t^{3}+\frac{1}{a^{3}}\right)}} d t=\int_{1}^{\infty} \frac{1+a^{2} t^{2}}{\sqrt{t\left(t^{3}-1\right)\left(a^{3} t^{3}+\frac{1}{a^{3}}\right)}} d t \\
& \sqrt{3} \int_{1}^{\infty} \frac{1-a^{2} t^{2}}{\sqrt{t\left(t^{3}-1\right)\left(a^{3} t^{3}+\frac{1}{a^{3}}\right)}} d t=-\int_{0}^{1} \frac{1+a^{2} t^{2}}{\sqrt{t\left(1-t^{3}\right)\left(a^{3} t^{3}+\frac{1}{a^{3}}\right)}} d t .
\end{aligned}
$$

Next we shall show that $j\left(C_{1}\right) \cup C_{2}$ is homotopic to $j\left(\varphi\left(C_{3}\right)\right) \cup \varphi\left(C_{4}\right)$. Choosing suitable $m, n \in\{0,1\}$, we obtain that $j\left(C_{1}\right) \cup C_{2}$ is homotopic to $j^{m}\left(\varphi\left(C_{3}\right)\right) \cup$ $j^{n}\left(\varphi\left(C_{4}\right)\right)$. The equation $\int_{j\left(C_{1}\right) \cup C_{2}} \frac{2 z}{w} d z=\int_{j^{m}\left(\varphi\left(C_{3}\right)\right) \cup j^{n}\left(\varphi\left(C_{4}\right)\right)} \frac{2 z}{w} d z$ yields that $m=1$ and $n=0$. Hence we have the following figure.
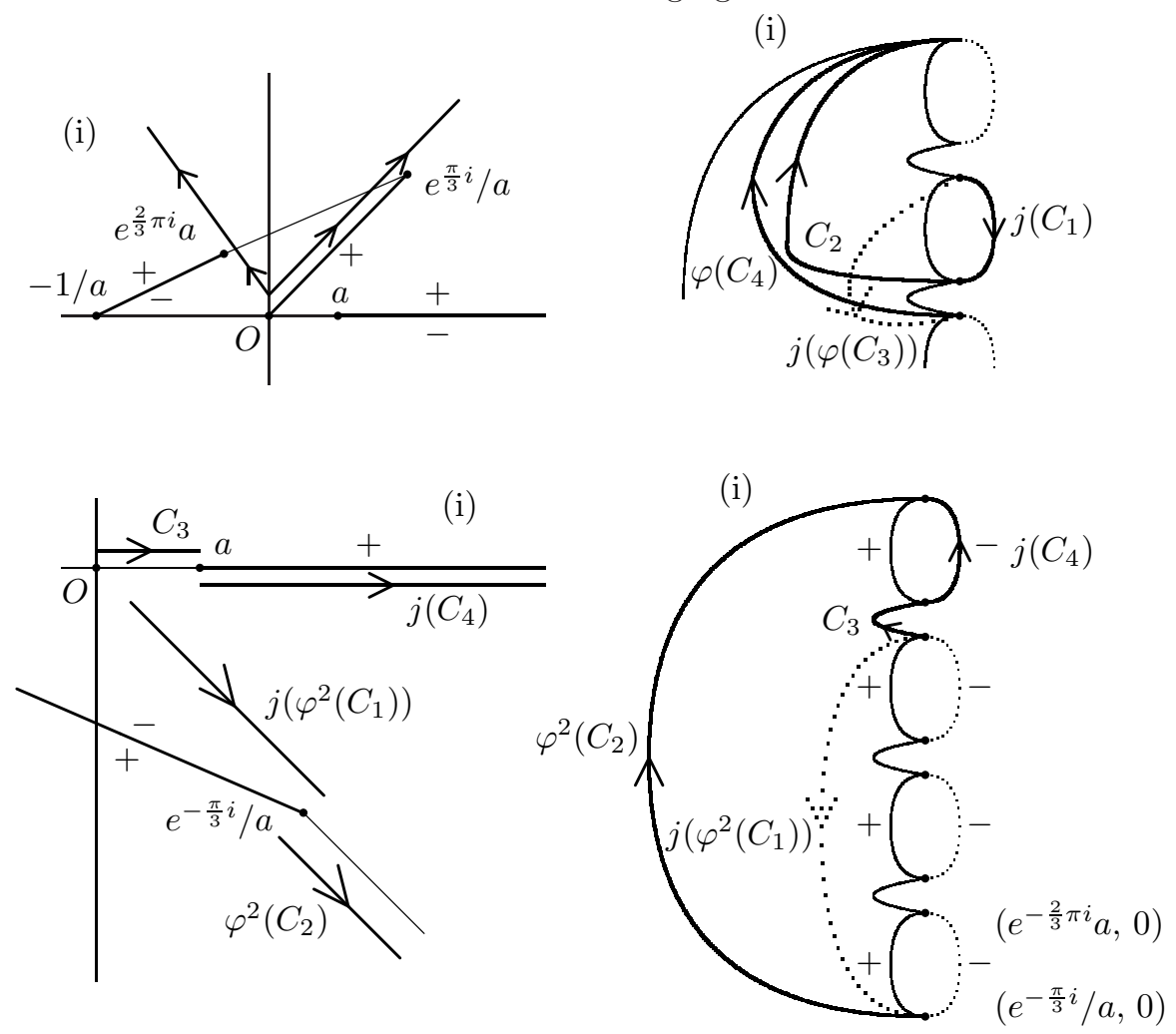

Moreover, it follows from $j\left(C_{1}\right) \cup C_{2} \sim j\left(\varphi\left(C_{3}\right)\right) \cup \varphi\left(C_{4}\right)$ that $j\left(\varphi^{2}\left(C_{1}\right)\right) \cup$ $\varphi^{2}\left(C_{2}\right) \sim C_{3} \cup j\left(C_{4}\right)$ and $\varphi\left(C_{1}\right) \cup j\left(\varphi\left(C_{2}\right)\right) \sim \varphi^{2}\left(C_{3}\right) \cup j\left(\varphi^{2}\left(C_{4}\right)\right)$. From the first relation, we find the above figure.

We shall write an accurate figure from the second relation. Choosing a suitable $m \in\{0,1\}$, we obtain the following figure. 


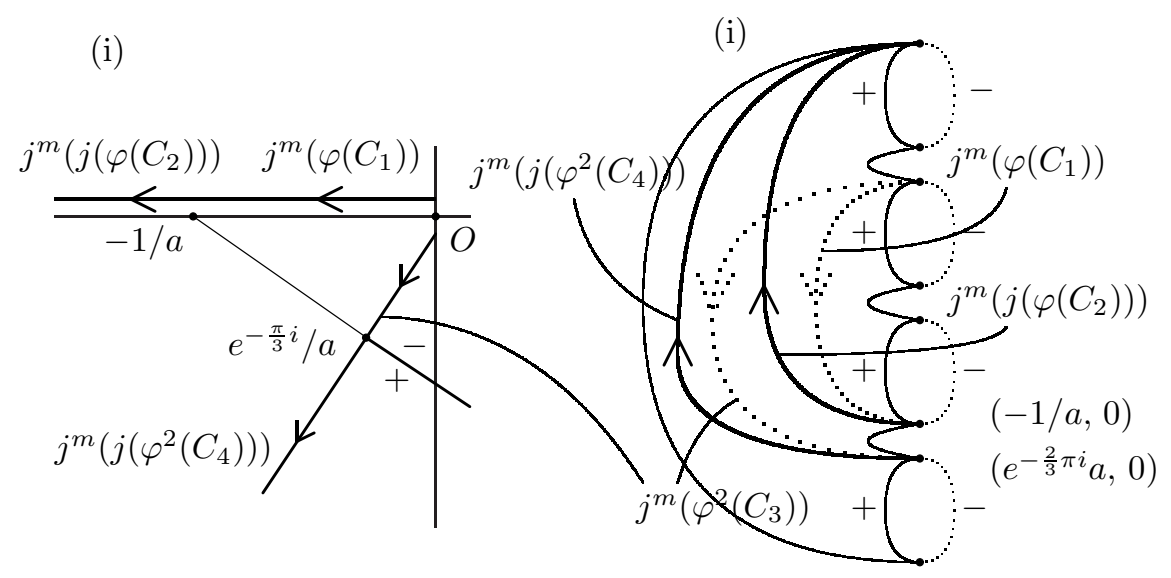

Thus we have $j^{m}\left(j\left(\varphi^{2}\left(C_{4}\right)\right)\right) \cup\left\{-C_{4}\right\} \cup\left\{-j\left(C_{3}\right)\right\} \cup j\left(j^{m}\left(\varphi^{2}\left(C_{3}\right)\right)\right) \sim \varphi^{2}\left(C_{2}\right) \cup$ $\left\{-j\left(\varphi^{2}\left(C_{2}\right)\right)\right\}$. The equation $\int_{j^{m}\left(j\left(\varphi^{2}\left(C_{4}\right)\right)\right) \cup\left\{-C_{4}\right\} \cup\left\{-j\left(C_{3}\right)\right\} \cup j\left(j^{m}\left(\varphi^{2}\left(C_{3}\right)\right)\right)} \frac{2 z}{w} d z=$ $\int_{\varphi^{2}\left(C_{2}\right) \cup\left\{-j\left(\varphi^{2}\left(C_{2}\right)\right)\right.} \frac{2 z}{w} d z$ yields $m=1$.

Therefore, we find a canonical homology basis as follows.

$$
\begin{aligned}
& A_{3}=\varphi\left(C_{1}\right)-j\left(\varphi\left(C_{1}\right)\right)-\varphi^{2}\left(C_{3}\right)+j\left(\varphi^{2}\left(C_{3}\right)\right), \\
& B_{3}=-\varphi^{2}\left(C_{1}\right)+j\left(\varphi^{2}\left(C_{1}\right)\right)+\varphi^{2}\left(C_{3}\right)-j\left(\varphi^{2}\left(C_{3}\right)\right), \\
& A_{2}=C_{1}-j\left(C_{1}\right)-\varphi\left(C_{3}\right)+j\left(\varphi\left(C_{3}\right)\right), \\
& B_{2}=-\varphi\left(C_{1}\right)+j\left(\varphi\left(C_{1}\right)\right)+\varphi\left(C_{3}\right)-j\left(\varphi\left(C_{3}\right)\right)+B_{3}, \\
& A_{1}=-C_{3}+j\left(C_{3}\right), \quad B_{1}=-C_{1}+j\left(C_{1}\right)+B_{2} .
\end{aligned}
$$

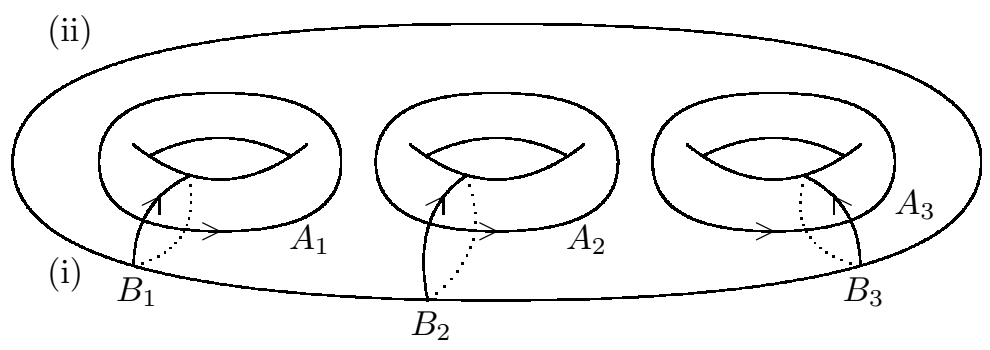

\subsubsection{Period matrix}

Key 1-cycles of the canonical homology basis as in $\S 5.2 .1$ are given by $C_{1} \cup$ $\left\{-j\left(C_{1}\right)\right\}$ and $C_{3} \cup\left\{-j\left(C_{3}\right)\right\}$. First, we have from (5.23) and (5.24):

$$
\int_{C_{1}} \frac{i\left(1+z^{2}\right)}{w} d z=\frac{1}{2 a} \int_{0}^{1} \frac{1+a^{2} t^{2}}{\sqrt{t\left(1-t^{3}\right)\left(a^{3} t^{3}+\frac{1}{a^{3}}\right)}} d t+\frac{i}{2 a} \int_{1}^{\infty} \frac{1+a^{2} t^{2}}{\sqrt{t\left(t^{3}-1\right)\left(a^{3} t^{3}+\frac{1}{a^{3}}\right)}} d t
$$




$$
\int_{C_{3}} \frac{i\left(1+z^{2}\right)}{w} d z=\frac{1}{a} \int_{0}^{1} \frac{1+a^{2} t^{2}}{\sqrt{t\left(1-t^{3}\right)\left(a^{3} t^{3}+\frac{1}{a^{3}}\right)}} d t .
$$

Thus, by (5.19)-(5.22), (5.25), and (5.26), we find

$$
\begin{gathered}
\int_{C_{1} \cup\left\{-j\left(C_{1}\right)\right\}} \frac{1-z^{2}}{w} d z=-\frac{\sqrt{3}}{a} i \int_{1}^{\infty} \frac{1+a^{2} t^{2}}{\sqrt{t\left(t^{3}-1\right)\left(a^{3} t^{3}+\frac{1}{a^{3}}\right)}} d t \\
+\frac{1}{\sqrt{3} a} \int_{0}^{1} \frac{1+a^{2} t^{2}}{\sqrt{t\left(1-t^{3}\right)\left(a^{3} t^{3}+\frac{1}{a^{3}}\right)}} d t, \\
\int_{C_{3} \cup\left\{-j\left(C_{3}\right)\right\}} \frac{1-z^{2}}{w} d z=-\frac{2}{\sqrt{3} a} i \int_{1}^{\infty} \frac{1+a^{2} t^{2}}{\sqrt{t\left(t^{3}-1\right)\left(a^{3} t^{3}+\frac{1}{a^{3}}\right)}} d t \\
\int_{C_{1} \cup\left\{-j\left(C_{1}\right)\right\}} \frac{i\left(1+z^{2}\right)}{w} d z=\frac{1}{a} \int_{0}^{1} \frac{1+a^{2} t^{2}}{\sqrt{t\left(1-t^{3}\right)\left(a^{3} t^{3}+\frac{1}{a^{3}}\right)}} d t \\
+\frac{i}{a} \int_{1}^{\infty} \frac{1+a^{2} t^{2}}{\sqrt{t\left(t^{3}-1\right)\left(a^{3} t^{3}+\frac{1}{a^{3}}\right)}} d t, \\
\int_{C_{3} \cup\left\{-j\left(C_{3}\right)\right\}} \frac{1\left(1+z^{2}\right)}{w} d z=\frac{2}{a} \int_{0}^{1} \frac{1+a^{2} t^{2}}{\sqrt{t\left(1-t^{3}\right)\left(a^{3} t^{3}+\frac{1}{a^{3}}\right)}} d t, \\
\int_{C_{1} \cup\left\{-j\left(C_{1}\right)\right\}} \frac{2 z}{w} d z=4 \int_{1}^{\infty} \frac{t}{\sqrt{t\left(t^{3}-1\right)\left(a^{3} t^{3}+\frac{1}{a^{3}}\right)}} d t \\
\int_{C_{3} \cup\left\{-j\left(C_{3}\right)\right\}} \frac{2 z}{w} d z=-4 i \int_{0}^{1} \frac{t}{\sqrt{t\left(1-t^{3}\right)\left(a^{3} t^{3}+\frac{1}{a^{3}}\right)}} d t .
\end{gathered}
$$

Next, we calculate periods of the meromorphic differentials with poles. From now on, let $\gamma$ be an arbitrary 1-cycle on $M$.

Substituting $\alpha=4, \beta=-1$ to (3.10) yields

$$
\begin{aligned}
& \int_{\gamma} \frac{z^{4}}{w^{3}} d z=\frac{1}{6} \int_{\gamma} \frac{z^{3}}{w} d z-\frac{1}{2}\left(a^{3}-\frac{1}{a^{3}}\right) \int_{\gamma} \frac{z^{7}}{w^{3}} d z \\
& \underbrace{=} \frac{1}{6} \int_{\gamma} \frac{z^{3}}{w} d z-\frac{1}{2}\left(a^{3}-\frac{1}{a^{3}}\right)\left\{\frac{1}{6} \int_{\gamma} \frac{d z}{w}+\frac{1}{2}\left(a^{3}-\frac{1}{a^{3}}\right) \int_{\gamma} \frac{z^{4}}{w^{3}} d z\right\} .
\end{aligned}
$$

Thus we find

$$
\int_{\gamma} \frac{z^{4}}{w^{3}} d z=\frac{a^{6}}{3\left(a^{6}+1\right)^{2}}\left\{2 \int_{\gamma} \frac{z^{3}}{w} d z-\left(a^{3}-\frac{1}{a^{3}}\right) \int_{\gamma} \frac{d z}{w}\right\} .
$$

Substituting $\alpha=5, \beta=-1$ to (3.10) implies

$$
\int_{\gamma} \frac{z^{5}}{w^{3}} d z=\frac{1}{2} \int_{\gamma} \frac{z^{4}}{w} d z-\frac{1}{2}\left(a^{3}-\frac{1}{a^{3}}\right) \int_{\gamma} \frac{z^{8}}{w^{3}} d z
$$




$$
\begin{aligned}
& \underbrace{=}_{\text {(3.17) }} \frac{1}{2} \int_{\gamma} \frac{z^{4}}{w} d z+\frac{1}{2}\left(a^{3}-\frac{1}{a^{3}}\right) \int_{\gamma} \frac{z^{2}}{w^{3}} d z \\
& \underbrace{=}_{(3.13)} \frac{1}{2} \int_{\gamma} \frac{z^{4}}{w} d z+\frac{1}{2}\left(a^{3}-\frac{1}{a^{3}}\right)\left\{-\frac{1}{2} \int_{\gamma} \frac{z}{w} d z+\frac{1}{2}\left(-a^{3}+\frac{1}{a^{3}}\right) \int_{\gamma} \frac{z^{5}}{w^{3}} d z\right\} .
\end{aligned}
$$

Hence we have

$$
\int_{\gamma} \frac{z^{5}}{w^{3}} d z=\frac{a^{6}}{\left(a^{6}+1\right)^{2}}\left\{2 \int_{\gamma} \frac{z^{4}}{w} d z-\left(a^{3}-\frac{1}{a^{3}}\right) \int_{\gamma} \frac{z}{w} d z\right\} .
$$

Substituting $\alpha=6, \beta=-1$ to (3.10) implies

$$
\begin{aligned}
& \int_{\gamma} \frac{z^{6}}{w^{3}} d z=\frac{5}{6} \int_{\gamma} \frac{z^{5}}{w} d z-\frac{1}{2}\left(a^{3}-\frac{1}{a^{3}}\right) \int_{\gamma} \frac{z^{9}}{w^{3}} d z \\
& \underbrace{=}_{\underbrace{3.18}_{\gamma}} \frac{5}{6} \int_{\gamma} \frac{z^{5}}{w} d z-\frac{1}{2}\left(a^{3}-\frac{1}{a^{3}}\right)\left\{\frac{5}{6} \int_{\gamma} \frac{z^{2}}{w} d z+\frac{1}{2}\left(a^{3}-\frac{1}{a^{3}}\right) \int_{\gamma} \frac{z^{6}}{w^{3}} d z\right\} .
\end{aligned}
$$

So we obtain

$$
\int_{\gamma} \frac{z^{6}}{w^{3}} d z=\frac{5 a^{6}}{3\left(a^{6}+1\right)^{2}}\left\{2 \int_{\gamma} \frac{z^{5}}{w} d z-\left(a^{3}-\frac{1}{a^{3}}\right) \int_{\gamma} \frac{z^{2}}{w} d z\right\} .
$$

From (5.33), (5.34), and (5.35), we find

$$
\begin{aligned}
& \int_{C_{1}-j\left(C_{1}\right)} \frac{z^{4}}{w^{3}} d z=\frac{2 e^{-\frac{\pi}{3} i} a^{4}}{3\left(a^{6}+1\right)^{2}} \int_{0}^{1} \frac{-2 t^{3}+1-a^{6}}{\sqrt{t\left(1-t^{3}\right)\left(a^{3}+\frac{t^{3}}{a^{3}}\right)}} d t, \\
& \int_{C_{3}-j\left(C_{3}\right)} \frac{z^{4}}{w^{3}} d z=-\frac{2 i a^{2}}{3\left(a^{6}+1\right)^{2}} \int_{0}^{1} \frac{2 a^{6} t^{3}+1-a^{6}}{\sqrt{t\left(1-t^{3}\right)\left(a^{3} t^{3}+\frac{1}{a^{3}}\right)}} d t, \\
& \int_{C_{1}-j\left(C_{1}\right)} \frac{z^{5}}{w^{3}} d z=\frac{2 a^{3}}{\left(a^{6}+1\right)^{2}} \int_{0}^{1} \frac{-2 t^{4}+\left(1-a^{6}\right) t}{\sqrt{t\left(1-t^{3}\right)\left(a^{3}+\frac{t^{3}}{a^{3}}\right)}} d t \\
& \int_{C_{3}-j\left(C_{3}\right)} \frac{z^{5}}{w^{3}} d z=-\frac{2 i a^{3}}{\left(a^{6}+1\right)^{2}} \int_{0}^{1} \frac{2 a^{6} t^{4}+\left(1-a^{6}\right) t}{\sqrt{t\left(1-t^{3}\right)\left(a^{3} t^{3}+\frac{1}{a^{3}}\right)}} d t, \\
& \int_{C_{1}-j\left(C_{1}\right)} \frac{z^{6}}{w^{3}} d z=\frac{10 e^{-\frac{2}{3} \pi i} a^{2}}{3\left(a^{6}+1\right)^{2}} \int_{0}^{1} \frac{2 t^{5}+\left(a^{6}-1\right) t^{2}}{\sqrt{t\left(1-t^{3}\right)\left(a^{3}+\frac{t^{3}}{a^{3}}\right)}} d t, \\
& \int_{C_{3}-j\left(C_{3}\right)} \frac{z^{6}}{w^{3}} d z=-\frac{10 i a^{4}}{3\left(a^{6}+1\right)^{2}} \int_{0}^{1} \frac{2 a^{6} t^{5}+\left(1-a^{6}\right) t^{2}}{\sqrt{t\left(1-t^{3}\right)\left(a^{3} t^{3}+\frac{1}{a^{3}}\right)}} d t .
\end{aligned}
$$

Now, by using the relation $C_{1} \cup C_{2} \sim C_{3} \cup C_{4}$, we consider relations between the above path-integrals. Note that $M$ can be constructed via a glueing the 
following two curves by the relation $x=-1 / z, y=w / z^{4}$ :

$$
w^{2}=z\left(z^{3}-a^{3}\right)\left(z^{3}+\frac{1}{a^{3}}\right) \underbrace{\longleftrightarrow}_{x=-\frac{1}{z}, y=\frac{w}{z^{4}}} y^{2}=x\left(x^{3}-a^{3}\right)\left(x^{3}+\frac{1}{a^{3}}\right) .
$$

So $C_{2}$ and $C_{4}$ can be rewritten as

$C_{2}=\left\{(x, y)=\left(-a e^{-\frac{\pi}{3} i} t, a^{2} e^{-\frac{\pi}{6} i} \sqrt{t\left(1-t^{3}\right)\left(a^{3} t^{3}+1 / a^{3}\right)}\right) \mid t: 1 \rightarrow 0, \sqrt{*}>0\right\}$,

$C_{4}=\left\{(x, y)=\left(-t / a, \sqrt{t\left(1-t^{3}\right)\left(a^{3}+t^{3} / a^{3}\right)} / a^{2}\right) \mid t: 1 \rightarrow 0, \sqrt{*}>0\right\}$.

Also, we have Lemma 3.3 and Lemma 3.4 in the coordinates $(x, y)$. Hence, by (5.33), (5.34), and (5.35), we find

$$
\begin{aligned}
& \int_{C_{2}-j\left(C_{2}\right)} \frac{z^{4}}{w^{3}} d z=\int_{C_{2}-j\left(C_{2}\right)} \frac{x^{6}}{y^{3}} d x=\frac{10 e^{\frac{\pi}{6} i} a^{4}}{3\left(a^{6}+1\right)^{2}} \int_{0}^{1} \frac{-2 a^{6} t^{5}+\left(a^{6}-1\right) t^{2}}{\sqrt{t\left(1-t^{3}\right)\left(a^{3} t^{3}+\frac{1}{a^{3}}\right)}} d t \\
& \int_{C_{4}-j\left(C_{4}\right)} \frac{z^{4}}{w^{3}} d z=\int_{C_{4}-j\left(C_{4}\right)} \frac{x^{6}}{y^{3}} d x=\frac{10 a^{2}}{3\left(a^{6}+1\right)^{2}} \int_{0}^{1} \frac{-2 t^{5}+\left(1-a^{6}\right) t^{2}}{\sqrt{t\left(1-t^{3}\right)\left(a^{3}+\frac{t^{3}}{a^{3}}\right)}} d t \\
& \int_{C_{2}-j\left(C_{2}\right)} \frac{z^{5}}{w^{3}} d z=-\int_{C_{2}-j\left(C_{2}\right)} \frac{x^{5}}{y^{3}} d x=-\frac{2 i a^{3}}{\left(a^{6}+1\right)^{2}} \int_{0}^{1} \frac{2 a^{6} t^{4}+\left(1-a^{6}\right) t}{\sqrt{t\left(1-t^{3}\right)\left(a^{3} t^{3}+\frac{1}{a^{3}}\right)}} d t, \\
& \int_{C_{4}-j\left(C_{4}\right)} \frac{z^{5}}{w^{3}} d z=-\int_{C_{4}-j\left(C_{4}\right)} \frac{x^{5}}{y^{3}} d x=\frac{2 a^{3}}{\left(a^{6}+1\right)^{2}} \int_{0}^{1} \frac{-2 t^{4}+\left(1-a^{6}\right) t}{\sqrt{t\left(1-t^{3}\right)\left(a^{3}+\frac{t^{3}}{a^{3}}\right)}} d t \\
& \int_{C_{2}-j\left(C_{2}\right)} \frac{z^{6}}{w^{3}} d z=\int_{C_{2}-j\left(C_{2}\right)} \frac{x^{4}}{y^{3}} d x=\frac{2 e^{-\frac{\pi}{6} i} a^{2}}{3\left(a^{6}+1\right)^{2}} \int_{0}^{1} \frac{2 a^{6} t^{3}+1-a^{6}}{\sqrt{t\left(1-t^{3}\right)\left(a^{3} t^{3}+\frac{1}{a^{3}}\right)}} d t \\
& \int_{C_{4}-j\left(C_{4}\right)} \frac{z^{6}}{w^{3}} d z=\int_{C_{4}-j\left(C_{4}\right)} \frac{x^{4}}{y^{3}} d x=\frac{2 a^{4}}{3\left(a^{6}+1\right)^{2}} \int_{0}^{1} \frac{-2 t^{3}+1-a^{6}}{\sqrt{t\left(1-t^{3}\right)\left(a^{3}+\frac{t^{3}}{a^{3}}\right)}} d t .
\end{aligned}
$$

Combining (5.36)-(5.47), and the relation $C_{1} \cup C_{2} \sim C_{3} \cup C_{4}$ yields

$$
\begin{aligned}
& -\frac{5}{2 \sqrt{3}} a^{2} \int_{0}^{1} \frac{2 a^{6} t^{5}+\left(1-a^{6}\right) t^{2}}{\sqrt{t\left(1-t^{3}\right)\left(a^{3} t^{3}+\frac{1}{a^{3}}\right)}} d t+\frac{1}{\sqrt{3}} \int_{0}^{1} \frac{2 a^{6} t^{3}+1-a^{6}}{\sqrt{t\left(1-t^{3}\right)\left(a^{3} t^{3}+\frac{1}{a^{3}}\right)}} d t \\
& =\frac{a^{2}}{2} \int_{0}^{1} \frac{-2 t^{3}+1-a^{6}}{\sqrt{t\left(1-t^{3}\right)\left(a^{3}+\frac{t^{3}}{a^{3}}\right)}} d t
\end{aligned}
$$




$$
\begin{aligned}
& -\frac{10}{\sqrt{3}} a^{2} \int_{0}^{1} \frac{2 a^{6} t^{5}+\left(1-a^{6}\right) t^{2}}{\sqrt{t\left(1-t^{3}\right)\left(a^{3} t^{3}+\frac{1}{a^{3}}\right)}} d t+\frac{1}{\sqrt{3}} \int_{0}^{1} \frac{2 a^{6} t^{3}+1-a^{6}}{\sqrt{t\left(1-t^{3}\right)\left(a^{3} t^{3}+\frac{1}{a^{3}}\right)}} d t \\
& =5 \int_{0}^{1} \frac{-2 t^{5}+\left(1-a^{6}\right) t^{2}}{\sqrt{t\left(1-t^{3}\right)\left(a^{3}+\frac{t^{3}}{a^{3}}\right)}} d t .
\end{aligned}
$$

By (5.36)-(5.41), (5.48), and (5.49), we have

$$
\begin{gathered}
\int_{C_{1} \cup\left\{-j\left(C_{1}\right)\right\}} \frac{z^{4}-z^{6}}{w^{3}} d z=\frac{a^{2}}{3\left(a^{6}+1\right)^{2}}\left\{\frac{1}{\sqrt{3}} \int_{0}^{1} \frac{\left(2 a^{6} t^{3}+1-a^{6}\right)\left(5 a^{2} t^{2}+1\right)}{\sqrt{t\left(1-t^{3}\right)\left(a^{3} t^{3}+\frac{1}{a^{3}}\right)}} d t\right. \\
\left.+3 i \int_{0}^{1} \frac{\left(2 a^{6} t^{3}+1-a^{6}\right)\left(5 a^{2} t^{2}-1\right)}{\sqrt{t\left(1-t^{3}\right)\left(a^{3} t^{3}+\frac{1}{a^{3}}\right)}} d t\right\}, \quad(5.50) \\
\int_{C_{3} \cup\left\{-j\left(C_{3}\right)\right\}} \frac{z^{4}-z^{6}}{w^{3}} d z=\frac{2 i a^{2}}{3\left(a^{6}+1\right)^{2}} \int_{0}^{1} \frac{\left(2 a^{6} t^{3}+1-a^{6}\right)\left(5 a^{2} t^{2}-1\right)}{\sqrt{t\left(1-t^{3}\right)\left(a^{3} t^{3}+\frac{1}{a^{3}}\right)}} d t \\
\int_{C_{1} \cup\left\{-j\left(C_{1}\right)\right\}} \frac{i\left(z^{4}+z^{6}\right)}{w^{3}} d z=\frac{a^{2}}{3\left(a^{6}+1\right)^{2}}\left\{\int_{0}^{1} \frac{\left(2 a^{6} t^{3}+1-a^{6}\right)\left(5 a^{2} t^{2}+1\right)}{\sqrt{t\left(1-t^{3}\right)\left(a^{3} t^{3}+\frac{1}{a^{3}}\right)}} d t\right. \\
-\sqrt{3 i} \int_{0}^{1} \frac{\left(2 a^{6} t^{3}+1-a^{6}\right)\left(5 a^{2} t^{2}-1\right)}{\left.\sqrt{t\left(1-t^{3}\right)\left(a^{3} t^{3}+\frac{1}{a^{3}}\right)} d t\right\},(5.52)} \\
\int_{C_{3} \cup\left\{-j\left(C_{3}\right)\right\}} \frac{\left.2 z^{4}+z^{6}\right)}{w^{3}} d z=\frac{2 a^{2}}{3\left(a^{6}+1\right)^{2}} \int_{0}^{1} \frac{\left(2 a^{6} t^{3}+1-a^{6}\right)\left(5 a^{2} t^{2}+1\right)}{\sqrt{t\left(1-t^{3}\right)\left(a^{3} t^{3}+\frac{1}{a^{3}}\right)}} d t \\
\int_{C_{1} \cup\left\{-j\left(C_{1}\right)\right\}} \frac{z^{5}}{w^{3}} d z= \\
\int_{C_{3} \cup\left\{-j\left(C_{3}\right)\right\}} \frac{2 a^{3}}{\left(a^{6}+1\right)^{2}} \int_{0}^{1} \frac{-2 t^{4}+\left(1-a^{6}\right) t}{\sqrt{t\left(1-t^{3}\right)\left(a^{3}+\frac{t^{3}}{a^{3}}\right)} d t} d z=-\frac{2 i a^{3}}{\left(a^{6}+1\right)^{2}} \int_{0}^{1} \frac{2 a^{6} t^{4}+\left(1-a^{6}\right) t}{\sqrt{t\left(1-t^{3}\right)\left(a^{3} t^{3}+\frac{1}{a^{3}}\right)}} d t . \quad(5.55)
\end{gathered}
$$

Set

$$
\begin{aligned}
& A=\frac{1}{\sqrt{3} a} \int_{0}^{1} \frac{1+a^{2} t^{2}}{\sqrt{t\left(1-t^{3}\right)\left(a^{3} t^{3}+\frac{1}{a^{3}}\right)}} d t, B=\frac{1}{\sqrt{3} a} \int_{1}^{\infty} \frac{1+a^{2} t^{2}}{\sqrt{t\left(t^{3}-1\right)\left(a^{3} t^{3}+\frac{1}{a^{3}}\right)}} d t \\
& C=4 \int_{1}^{\infty} \frac{t}{\sqrt{t\left(t^{3}-1\right)\left(a^{3} t^{3}+\frac{1}{a^{3}}\right)}} d t, D=4 \int_{0}^{1} \frac{t}{\sqrt{t\left(1-t^{3}\right)\left(a^{3} t^{3}+\frac{1}{a^{3}}\right)}} d t \\
& E=\frac{a^{2}}{3 \sqrt{3}\left(a^{6}+1\right)^{2}} \int_{0}^{1} \frac{\left(2 a^{6} t^{3}+1-a^{6}\right)\left(5 a^{2} t^{2}+1\right)}{\sqrt{t\left(1-t^{3}\right)\left(a^{3} t^{3}+\frac{1}{a^{3}}\right)}} d t
\end{aligned}
$$




$$
\begin{aligned}
& F=\frac{a^{2}}{3\left(a^{6}+1\right)^{2}} \int_{0}^{1} \frac{\left(2 a^{6} t^{3}+1-a^{6}\right)\left(5 a^{2} t^{2}-1\right)}{\sqrt{t\left(1-t^{3}\right)\left(a^{3} t^{3}+\frac{1}{a^{3}}\right)}} d t \\
& H=\frac{2 a^{3}}{\left(a^{6}+1\right)^{2}} \int_{0}^{1} \frac{-2 t^{4}+\left(1-a^{6}\right) t}{\sqrt{t\left(1-t^{3}\right)\left(a^{3}+\frac{t^{3}}{a^{3}}\right)}} d t, I=\frac{2 a^{3}}{\left(a^{6}+1\right)^{2}} \int_{0}^{1} \frac{2 a^{6} t^{4}+\left(1-a^{6}\right) t}{\sqrt{t\left(1-t^{3}\right)\left(a^{3} t^{3}+\frac{1}{a^{3}}\right)}} d t .
\end{aligned}
$$

From (5.27)-(5.32), (5.50)-(5.55), we find

$$
\int_{C_{1} \cup\left\{-j\left(C_{1}\right)\right\}} G=i\left(\begin{array}{c}
A-3 i B \\
\sqrt{3}(A+i B) \\
C \\
E+3 i F \\
\sqrt{3}(E-i F) \\
H
\end{array}\right), \quad \int_{C_{3} \cup\left\{-j\left(C_{3}\right)\right\}} G=i\left(\begin{array}{c}
-2 i B \\
2 \sqrt{3} A \\
-i D \\
2 i F \\
2 \sqrt{3} E \\
-i I
\end{array}\right) .
$$

Therefore, the period matrix of the abelian differentials of the second kind is given by

$$
i\left(\begin{array}{cccccc}
2 i B & -2(A+i B) & -(A+i B) & 2 A & 3(A-i B) & 2(A-i B) \\
-2 \sqrt{3} A & 0 & \sqrt{3}(A+i B) & -2 \sqrt{3} i B & \sqrt{3}(A-i B) & 0 \\
i D & C-i D & -C+i D & -C & 0 & -(C+i D) \\
-2 i F & 2(-E+i F) & -E+i F & 2 E & 3(E+i F) & 2(E+i F) \\
-2 \sqrt{3} E & 0 & \sqrt{3}(E-i F) & 2 \sqrt{3} i F & \sqrt{3}(E+i F) & 0 \\
i I & H-i I & -H+i I & -H & 0 & -(H+i I)
\end{array}\right) .
$$

\section{3 tP family, tD family}

\subsubsection{Canonical homology basis}

Let $M$ be a hyperelliptic Riemann surface of genus 3 defined as the completion of $\left\{(z, w) \mid w^{2}=z^{8}+a z^{4}+1\right\} \subset \mathbb{C}^{2}$ for $a \in(-\infty,-2) \cup(2, \infty)$. It suffices to consider the case $a \in(2, \infty)$ because we obtain the same result for $a \in$ $(-\infty,-2)$. The three differentials

$$
\frac{d z}{w}, z \frac{d z}{w}, z^{2} \frac{d z}{w}
$$

form a basis for the abelian differentials of the first kind. Up to exact forms, the abelian differentials of the second kind are given by the following six differentials:

$$
\frac{d z}{w}, z \frac{d z}{w}, z^{2} \frac{d z}{w}, \frac{z^{4}}{w^{3}} d z, \frac{z^{5}}{w^{3}} d z, \frac{z^{6}}{w^{3}} d z .
$$

Let

$$
G=\left(\frac{1-z^{2}}{w}, \frac{i\left(1+z^{2}\right)}{w}, \frac{2 z}{w}, \frac{z^{4}-z^{6}}{w^{3}}, \frac{i\left(z^{4}+z^{6}\right)}{w^{3}}, \frac{z^{5}}{w^{3}}\right)^{t} d z
$$


and consider the biholomorphisms

$$
j(z, w)=(z,-w), \varphi(z, w)=(i z, w)
$$

on $M$. Then it is straightforward to compute that

$$
j^{*} G=-G, \quad \varphi^{*} G=\left(\begin{array}{cccccc}
0 & 1 & 0 & 0 & 0 & 0 \\
-1 & 0 & 0 & 0 & 0 & 0 \\
0 & 0 & -1 & 0 & 0 & 0 \\
0 & 0 & 0 & 0 & 1 & 0 \\
0 & 0 & 0 & -1 & 0 & 0 \\
0 & 0 & 0 & 0 & 0 & -1
\end{array}\right) G
$$

Now we determine a canonical homology basis on $M$. Recall that

$$
\begin{gathered}
\pi_{\mathrm{tP}}: \quad M \longrightarrow \overline{\mathbb{C}}:=\mathbb{C} \cup\{\infty\} \\
(z, w) \longmapsto z
\end{gathered}
$$

defines a two-sheeted branched covering and $j$ is its deck transformation. Set $\alpha:=\sqrt{\frac{\sqrt{a+2}+\sqrt{a-2}}{2}}>1 . \pi_{\mathrm{tP}}$ has branch locus

$$
\left\{\alpha e^{ \pm \frac{\pi}{4} i}, \alpha e^{ \pm \frac{3}{4} \pi i}, \alpha^{-1} e^{ \pm \frac{\pi}{4} i}, \alpha^{-1} e^{ \pm \frac{3}{4} \pi i}\right\}
$$
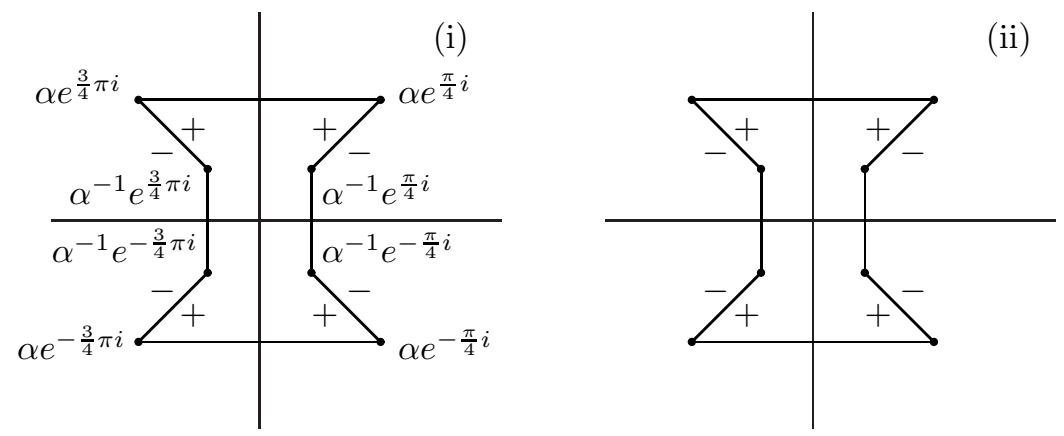

\section{figure (tP)}

So $M$ can be expressed as a 2 -sheeted branched cover of $\overline{\mathbb{C}}$ as the above. We prepare two copies of $\overline{\mathbb{C}}$ and slit them along the thick lines in figure (tP). Identifying each of the upper (resp. lower) edges of the thick lines in (i) with each of the lower (resp. upper) edges of the thick lines in (ii), we obtain the hyperelliptic Riemann surface $M$ of genus 3 (see the following figure). Note that each of thin lines joining two branch points in figure (tP) is corresponding to each of thick lines joining two branch points in the following figure. 


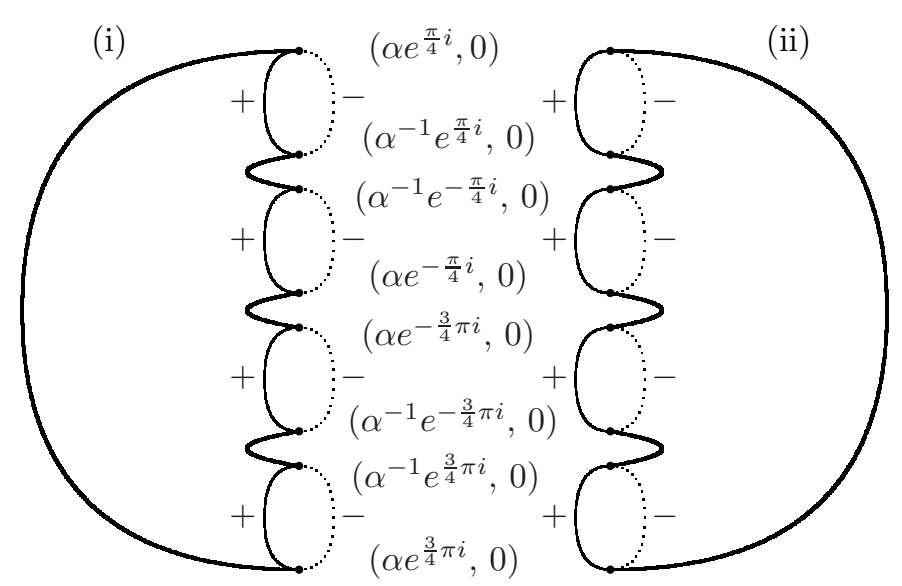

To describe 1-cycles on $M$, we consider the following key 1-cycles:

$$
\begin{gathered}
C_{1}=\left\{(z, w)=\left(t, \sqrt{t^{8}+a t^{4}+1}\right) \mid t: 0 \rightarrow \infty, \sqrt{*}>0\right\} \\
\cup\left\{(z, w)=\left(i t, \sqrt{t^{8}+a t^{4}+1}\right) \mid t: \infty \rightarrow 0, \sqrt{*}>0\right\}, \\
C_{2}=\left\{(z, w)=\left(i t, \sqrt{t^{8}+a t^{4}+1}\right) \mid t: 1 \rightarrow-1, \sqrt{*}>0\right\} \\
\cup\left\{(z, w)=\left(e^{i t}, w(t)\right) \mid t:-\pi / 2 \rightarrow \pi / 2, w(0)<0\right\} .
\end{gathered}
$$

We shall verify that $C_{2}$ defines a connected 1 -cycle later. Since $C_{1} \cap C_{2} \neq \varnothing$, we may choose $C_{1}$ and $C_{2}$ in the following figure.
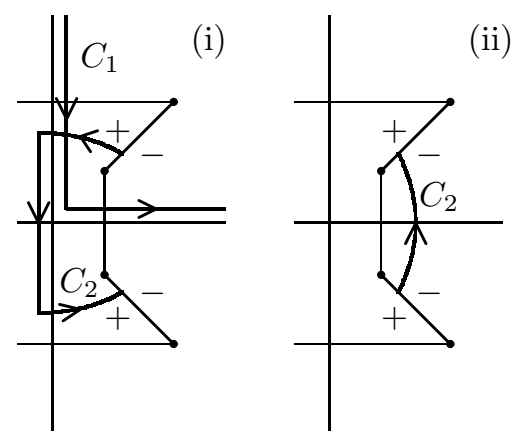

(ii)

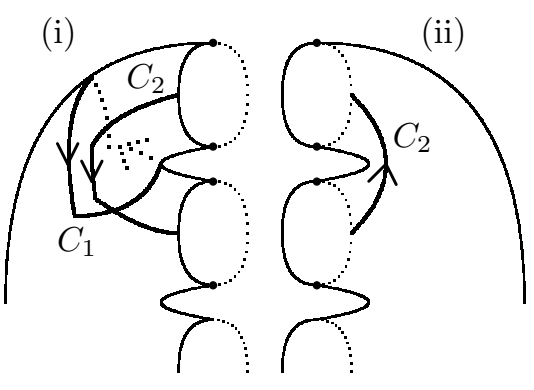

After that, we shall give other 1-cycles. From $C_{1} \cap \varphi^{2}\left(C_{1}\right) \neq \varnothing$ and $C_{1} \cap \varphi^{3}\left(C_{1}\right) \neq$ $\varnothing$, we have the following two figures. 


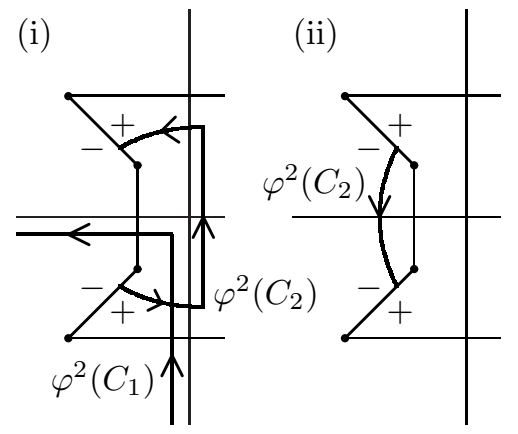

(i) (i)

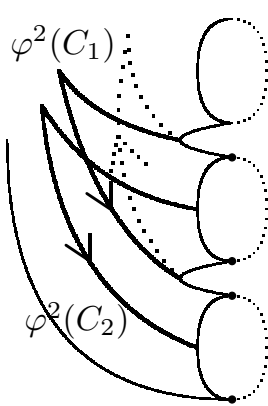

(i)

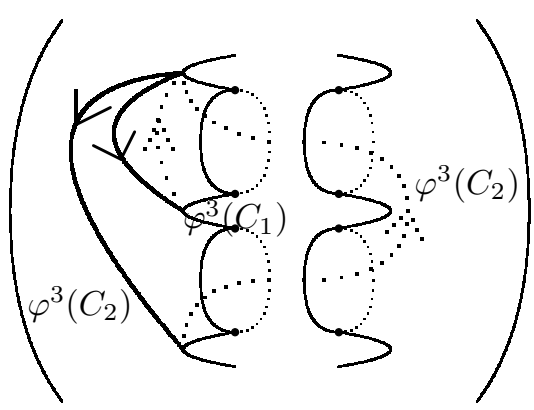

(ii)

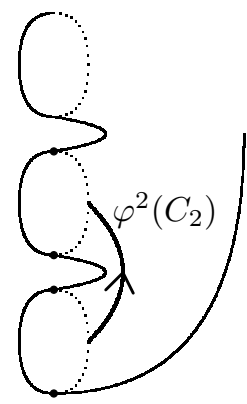

(ii)
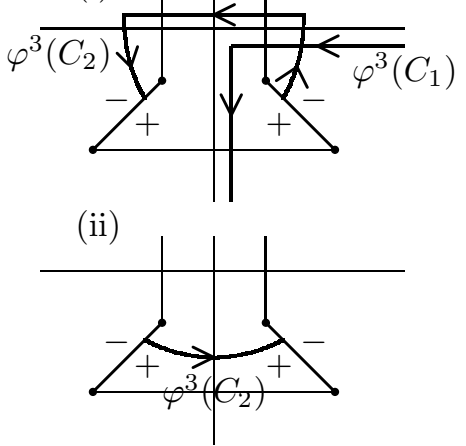

Therefore, we find a canonical homology basis as follows.

$$
\begin{aligned}
& A_{1}=C_{2}, \quad B_{1}=C_{1}, \quad A_{2}=-\varphi^{2}\left(C_{1}\right)-\varphi^{3}\left(C_{1}\right)+\varphi^{3}\left(C_{2}\right), \\
& B_{2}=B_{1}+\varphi^{3}\left(C_{1}\right), \quad A_{3}=\varphi^{2}\left(C_{2}\right), \quad B_{3}=B_{2}+\varphi^{2}\left(C_{1}\right) .
\end{aligned}
$$

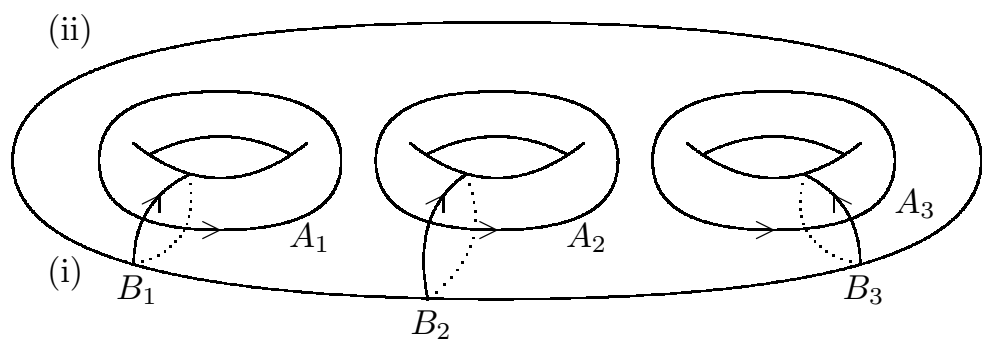

\subsubsection{Period matrix}

Key 1-cycles of the canonical homology basis as in $\S 5.3 .1$ are given by $C_{1}$ and $C_{2}$. First, we have

$$
\int_{C_{1}} \frac{1-z^{2}}{w} d z=-i \int_{0}^{\infty} \frac{1+t^{2}}{\sqrt{t^{8}+a t^{4}+1}} d t=-2 i \int_{0}^{1} \frac{1+t^{2}}{\sqrt{t^{8}+a t^{4}+1}} d t
$$




$$
\begin{aligned}
\int_{C_{1}} \frac{i\left(1+z^{2}\right)}{w} d z & =2 i \int_{0}^{1} \frac{1+t^{2}}{\sqrt{t^{8}+a t^{4}+1}} d t \\
\int_{C_{1}} \frac{2 z}{w} d z & =8 \int_{0}^{1} \frac{t}{\sqrt{t^{8}+a t^{4}+1}} d t \\
\int_{C_{1}} \frac{z^{4}-z^{6}}{w^{3}} d z & =-2 i \int_{0}^{1} \frac{t^{4}+t^{6}}{{\sqrt{t^{8}+a t^{4}+1}}^{3}} d t \\
\int_{C_{1}} \frac{i\left(z^{4}+z^{6}\right)}{w^{3}} d z & =2 i \int_{0}^{1} \frac{t^{4}+t^{6}}{{\sqrt{t^{8}+a t^{4}+1}}^{3}} d t \\
\int_{C_{1}} \frac{z^{5}}{w^{3}} d z & =4 \int_{0}^{1} \frac{t^{5}}{{\sqrt{t^{8}+a t^{4}+1}}^{3}} d t .
\end{aligned}
$$

Next we calculate periods along $C_{2}$. Set $x=(z+1 / z) / 2=\cos t: 0 \rightarrow 1 \rightarrow 0$ $(t:-\pi / 2 \rightarrow 0 \rightarrow \pi / 2)$ along a circle part of $C_{2}$. Then $\frac{1-z^{2}}{w} d z=-2 \frac{z^{2}}{w} d x$ and $\frac{z^{4}-z^{6}}{w^{3}} d z=-2\left(\frac{z^{2}}{w}\right)^{3} d x . z+1 / z=2 x$ implies that $z^{2}+1 / z^{2}=4 x^{2}-2$, and thus $z^{4}+1 / z^{4}=16 x^{4}-16 x^{2}+2$. Hence,

$$
\left(\frac{z^{2}}{w}\right)^{2}=\frac{z^{4}}{z^{8}+a z^{4}+1}=\frac{1}{z^{4}+\frac{1}{z^{4}}+a}=\frac{1}{16 x^{4}-16 x^{2}+2+a}>0 .
$$

To choose a suitable branch, we substitute $t=0$ to $z^{2} / w$. Then $\frac{z^{2}}{w}(t=0)=$ $\frac{1}{w(0)}<0$. As a result

$$
\frac{z^{2}}{w}=-\frac{1}{\sqrt{16 x^{4}-16 x^{2}+2+a}}<0 .
$$

Consequently, we have

$$
\begin{aligned}
\int_{C_{2}} \frac{1-z^{2}}{w} d z= & i \int_{1}^{-1} \frac{1+t^{2}}{\sqrt{t^{8}+a t^{4}+1}} d t+\int_{0}^{1} \frac{2}{\sqrt{16 x^{4}-16 x^{2}+2+a}} d x \\
& +\int_{1}^{0} \frac{2}{\sqrt{16 x^{4}-16 x^{2}+2+a}} d x \\
=- & 2 i \int_{0}^{1} \frac{1+t^{2}}{{\sqrt{t^{8}+a t^{4}+1}}^{-1}} d t \\
\int_{C_{2}} \frac{z^{4}-z^{6}}{w^{3}} d z= & i \int_{1}^{-1} \frac{t^{4}+t^{6}}{{\sqrt{t^{8}+a t^{4}+1}}^{3}} d t+\int_{0}^{1} \frac{2}{{\sqrt{16 x^{4}-16 x^{2}+2+a}}^{3}} d x \\
& +\int_{1}^{0} \frac{2}{{\sqrt{16 x^{4}-16 x^{2}+2+a}}^{3}} d t \\
=- & 2 i \int_{0}^{1} \frac{t^{4}+t^{6}}{{\sqrt{t^{8}+a t^{4}+1}}^{3}}
\end{aligned}
$$


Moreover, from (5.62) and substituting $t= \pm \pi / 2$ to $z^{2} / w$, we find $\frac{-1}{w( \pm \pi / 2)}<$ 0 . So $w( \pm \pi / 2)>0$. Thus, $C_{2}$ defines a connected 1-cycle.

We set $x=(z-1 / z) /(2 i)=\sin t:-1 \rightarrow 1(t:-\pi / 2 \rightarrow \pi / 2)$ along a circle part of $C_{2}$. Then $\frac{i\left(1+z^{2}\right)}{w} d z=-2 \frac{z^{2}}{w} d x$ and $\frac{i\left(z^{4}+z^{6}\right)}{w^{3}} d z=-2\left(\frac{z^{2}}{w}\right)^{3} d x$. $z-1 / z=2 i x$ implies that $z^{2}+1 / z^{2}=-4 x^{2}+2$, and thus $z^{4}+1 / z^{4}=$ $16 x^{4}-16 x^{2}+2$. Hence,

$$
\left(\frac{z^{2}}{w}\right)^{2}=\frac{z^{4}}{z^{8}+a z^{4}+1}=\frac{1}{z^{4}+\frac{1}{z^{4}}+a}=\frac{1}{16 x^{4}-16 x^{2}+2+a}>0 .
$$

To choose a suitable branch, we substitute $t=0$ to $z^{2} / w$. Then $\frac{z^{2}}{w}(t=0)=$ $\frac{1}{w(0)}<0$. As a result,

$$
\frac{z^{2}}{w}=-\frac{1}{\sqrt{16 x^{4}-16 x^{2}+2+a}}<0 .
$$

Consequently, we have

$$
\begin{aligned}
\int_{C_{2}} \frac{i\left(1+z^{2}\right)}{w} d z & =\int_{-1}^{1} \frac{1-t^{2}}{\sqrt{t^{8}+a t^{4}+1}} d t+\int_{-1}^{1} \frac{2}{\sqrt{16 x^{4}-16 x^{2}+2+a}} d x \\
& =2 \int_{0}^{1} \frac{1-t^{2}}{{\sqrt{t^{8}+a t^{4}+1}}^{1}} d t+4 \int_{0}^{1} \frac{d x}{\sqrt{16 x^{4}-16 x^{2}+2+a}} \\
\int_{C_{2}} \frac{i\left(z^{4}+z^{6}\right)}{w^{3}} d z & =\int_{-1}^{1} \frac{t^{4}-t^{6}}{{\sqrt{t^{8}+a t^{4}+1}}^{3}} d t+\int_{-1}^{1} \frac{2}{{\sqrt{16 x^{4}-16 x^{2}+2+a}}^{3}} d x \\
& =2 \int_{0}^{1} \frac{t^{4}-t^{6}}{{\sqrt{t^{8}+a t^{4}+1}}^{3}} d t+4 \int_{0}^{1} \frac{d x}{{\sqrt{16 x^{4}-16 x^{2}+2+a}}^{3}}
\end{aligned}
$$

Setting $x=\left(z^{2}-1\right) /\left(i\left(z^{2}+1\right)\right)=\tan t:-\infty \rightarrow \infty(t:-\pi / 2 \rightarrow \pi / 2)$ along a circle part of $C_{2}$, we find $\frac{2 z}{w} d z=\frac{i}{2} \frac{\left(z^{2}+1\right)^{2}}{w} d x$ and $\frac{z^{5}}{w^{3}} d z=\frac{i}{4} \frac{z^{4}}{w^{2}} \frac{\left(z^{2}+1\right)^{2}}{w} d x$. $z^{2}=\frac{1-x^{2}}{1+x^{2}}+i \frac{2 x}{1+x^{2}}$ implies that $z^{2}+1 / z^{2}=\frac{2\left(1-x^{2}\right)}{1+x^{2}}$, and thus $z^{4}+1 / z^{4}=$ $\frac{2 x^{4}-12 x^{2}+2}{\left(1+x^{2}\right)^{2}}$. Hence,

$$
\left(\frac{\left(z^{2}+1\right)^{2}}{w}\right)^{2}=\frac{16}{(2+a) x^{4}+(2 a-12) x^{2}+2+a}>0 .
$$


To choose a suitable branch, we substitute $t=0$ to $\left(z^{2}+1\right)^{2} / w$. Then $\frac{\left(z^{2}+1\right)^{2}}{w}(t=$ $0)=\frac{4}{w(0)}<0$. As a result,

$$
\frac{\left(z^{2}+1\right)^{2}}{w}=-\frac{4}{\sqrt{(2+a) x^{4}+(2 a-12) x^{2}+2+a}}<0 .
$$

Similarly, we obtain

$$
\frac{z^{4}}{w^{2}}=\frac{1}{z^{4}+\frac{1}{z^{4}}+a}=\frac{\left(1+x^{2}\right)^{2}}{(2+a) x^{4}+(2 a-12) x^{2}+2+a} .
$$

Consequently, we have

$$
\begin{aligned}
\int_{C_{2}} & \frac{2 z}{w} d z=\int_{-1}^{1} \frac{2 t}{\sqrt{t^{8}+a t^{4}+1}} d t-2 i \int_{-\infty}^{\infty} \frac{d x}{\sqrt{(2+a) x^{4}+(2 a-12) x^{2}+2+a}} \\
= & -8 i \int_{0}^{1} \frac{d x}{\sqrt{(2+a) x^{4}+(2 a-12) x^{2}+2+a}} \\
\int_{C_{2}} & \frac{z^{5}}{w^{3}} d z=\int_{-1}^{1} \frac{t^{5}}{{\sqrt{t^{8}+a t^{4}+1}}^{3}} d t-i \int_{-\infty}^{\infty} \frac{\left(1+x^{2}\right)^{2}}{{\sqrt{(2+a) x^{4}+(2 a-12) x^{2}+2+a}}^{3}} d x \\
= & -4 i \int_{0}^{1} \frac{\left(1+x^{2}\right)^{2}}{{\sqrt{(2+a) x^{4}+(2 a-12) x^{2}+2+a}}^{3}} d x .
\end{aligned}
$$

Set

$$
\begin{aligned}
& A=2 \int_{0}^{1} \frac{1-t^{2}}{\sqrt{t^{8}+a t^{4}+1}} d t+4 \int_{0}^{1} \frac{d t}{\sqrt{16 t^{4}-16 t^{2}+2+a}}, \\
& B=2 \int_{0}^{1} \frac{1+t^{2}}{\sqrt{t^{8}+a t^{4}+1}} d t, \quad C=8 \int_{0}^{1} \frac{t}{\sqrt{t^{8}+a t^{4}+1}} d t, \\
& D=8 \int_{0}^{1} \frac{d t}{\sqrt{(2+a) t^{4}+(2 a-12) t^{2}+2+a}}, \\
& E=2 \int_{0}^{1} \frac{t^{4}-t^{6}}{{\sqrt{t^{8}+a t^{4}+1^{3}}}^{3}} d t+4 \int_{0}^{1} \frac{d t}{{\sqrt{16 t^{4}-16 t^{2}+2+a}}^{3}}, \\
& F=2 \int_{0}^{1} \frac{t^{4}+t^{6}}{{\sqrt{t^{8}+a t^{4}+1}}^{3}} d t, \quad H=4 \int_{0}^{1} \frac{t^{5}}{{\sqrt{t^{8}+a t^{4}+1}}^{3}} d t, \\
& I=4 \int_{0}^{1} \frac{\left(1+t^{2}\right)^{2}}{{\sqrt{(2+a) t^{4}+(2 a-12) t^{2}+2+a}}^{3}} d t .
\end{aligned}
$$


By (5.56)-(5.61) and (5.63)-(5.68), we have

$$
\int_{C_{1}} G=\left(\begin{array}{c}
-i B \\
i B \\
C \\
-i F \\
i F \\
H
\end{array}\right), \quad \int_{C_{2}} G=\left(\begin{array}{c}
-i B \\
A \\
-i D \\
-i F \\
E \\
-i I
\end{array}\right)
$$

Therefore, the period matrix of the abelian differentials of the second kind is given by

$$
\left(\begin{array}{cccccc}
-i B & -A & i B & -i B & -2 i B & -i B \\
A & i B & -A & i B & 0 & -i B \\
-i D & i D & -i D & C & 0 & C \\
-i F & -E & i F & -i F & -2 i F & -i F \\
E & i F & -E & i F & 0 & -i F \\
-i I & i I & -i I & H & 0 & H
\end{array}\right) .
$$

\section{4 tCLP family}

\subsection{1 canonical homology basis}

Let $M$ be a hyperelliptic Riemann surface of genus 3 defined as the completion of $\left\{(z, w) \mid w^{2}=z^{8}+a z^{4}+1\right\} \subset \mathbb{C}^{2}$ for $a \in(-2,2)$. It suffices to consider the case $a \in[0,2)$ because we obtain the same result for $a \in(-2,0]$. The three differentials

$$
\frac{d z}{w}, z \frac{d z}{w}, z^{2} \frac{d z}{w}
$$

form a basis for the abelian differentials of the first kind. Up to exact forms, the abelian differentials of the second kind are given by the following six differentials:

$$
\frac{d z}{w}, z \frac{d z}{w}, z^{2} \frac{d z}{w}, \frac{z^{4}}{w^{3}} d z, \frac{z^{5}}{w^{3}} d z, \frac{z^{6}}{w^{3}} d z .
$$

Let

$$
G=\left(\frac{1-z^{2}}{w}, \frac{i\left(1+z^{2}\right)}{w}, \frac{2 z}{w}, \frac{z^{4}-z^{6}}{w^{3}}, \frac{i\left(z^{4}+z^{6}\right)}{w^{3}}, \frac{z^{5}}{w^{3}}\right)^{t} d z
$$

and consider the biholomorphisms

$$
j(z, w)=(z,-w), \varphi(z, w)=(i z, w)
$$

on $M$. Then it is straightforward to compute that

$$
j^{*} G=-G, \quad \varphi^{*} G=\left(\begin{array}{cccccc}
0 & 1 & 0 & 0 & 0 & 0 \\
-1 & 0 & 0 & 0 & 0 & 0 \\
0 & 0 & -1 & 0 & 0 & 0 \\
0 & 0 & 0 & 0 & 1 & 0 \\
0 & 0 & 0 & -1 & 0 & 0 \\
0 & 0 & 0 & 0 & 0 & -1
\end{array}\right) G
$$


Now we determine a canonical homology basis on $M$. Recall that

$$
\begin{gathered}
\pi_{\mathrm{tCLP}}: \quad M \longrightarrow \overline{\mathbb{C}}:=\mathbb{C} \cup\{\infty\} \\
(z, w) \longmapsto z
\end{gathered}
$$

defines a two-sheeted branched covering and $j$ is its deck transformation. Set $e^{i \alpha}:=-\frac{a}{2}+i \frac{\sqrt{4-a^{2}}}{2} \in S^{1} \subset \mathbb{C}(\alpha \in[\pi / 2, \pi)) . \pi_{\mathrm{tCLP}}$ has branch locus

$$
\left\{e^{ \pm \frac{\alpha}{4} i}, i e^{ \pm \frac{\alpha}{4} i},-e^{ \pm \frac{\alpha}{4} i},-i e^{ \pm \frac{\alpha}{4} i}\right\}
$$

So $M$ can be expressed as a 2 -sheeted branched cover of $\overline{\mathbb{C}}$ in the following way.
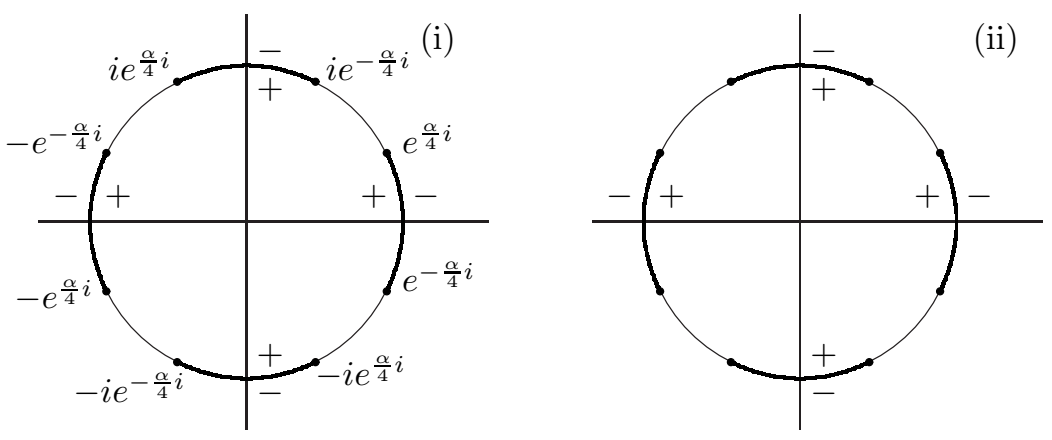

\section{figure (tCLP)}

We prepare two copies of $\overline{\mathbb{C}}$ and slit them along the thick lines in figure (tCLP). Identifying each of the upper (resp. lower) edges of the thick lines in (i) with each of the lower (resp. upper) edges of the thick lines in (ii), we obtain the hyperelliptic Riemann surface $M$ of genus 3 (see the following figure). Note that each of thin lines joining two branch points in figure (tCLP) is corresponding to each of thick lines joining two branch points in the following figure.

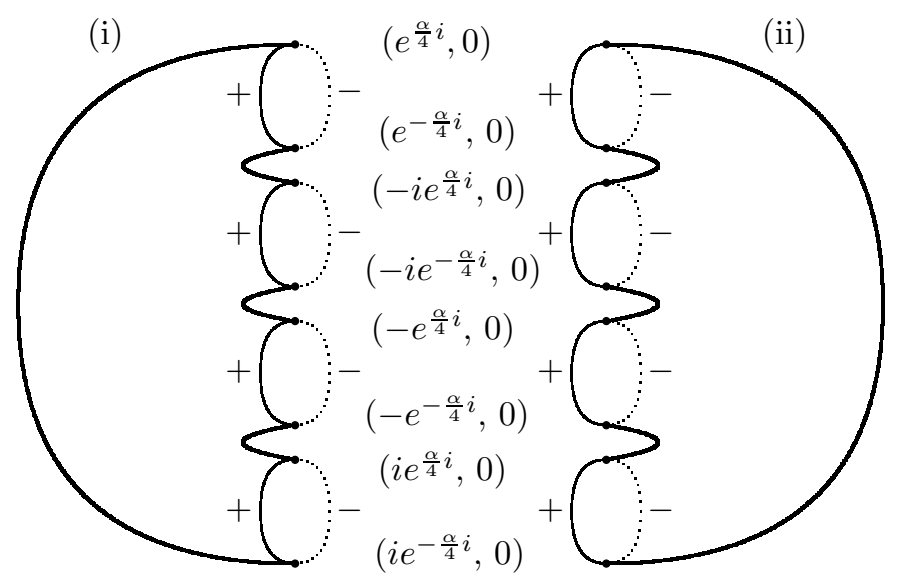


To describe 1-cycles on $M$, we consider the following key 1-cycles:

$$
\begin{gathered}
C_{1}=\left\{(z, w)=\left(t e^{\frac{\pi}{4} i}, \sqrt{t^{8}-a t^{4}+1}\right) \mid t: \infty \rightarrow 0, \sqrt{*}>0\right\} \\
\cup\left\{(z, w)=\left(t e^{-\frac{\pi}{4} i}, \sqrt{t^{8}-a t^{4}+1}\right) \mid t: 0 \rightarrow \infty, \sqrt{*}>0\right\}, \\
C_{2}=\left\{(z, w)=\left(t, \sqrt{t^{8}+a t^{4}+1}\right) \mid t: \infty \rightarrow 0, \sqrt{*}>0\right\} \\
\cup\left\{(z, w)=\left(-i t, \sqrt{t^{8}+a t^{4}+1}\right) \mid t: 0 \rightarrow \infty, \sqrt{*}>0\right\} .
\end{gathered}
$$

Since $C_{1} \cap C_{2} \neq \varnothing$, we may choose $C_{1}$ and $C_{2}$ in the following figure.
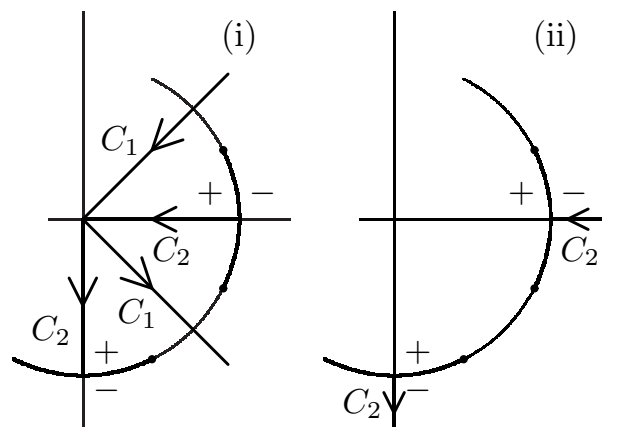

(i)

Next we shall consider other 1-cycles. From $C_{1} \cap\left(\varphi^{3}\left(C_{1}\right) \cap \varphi^{3}\left(C_{2}\right)\right) \neq \varnothing$ and $C_{1} \cap\left(\varphi^{2}\left(C_{1}\right) \cap \varphi^{2}\left(C_{2}\right)\right) \neq \varnothing$, we have the following two figures.

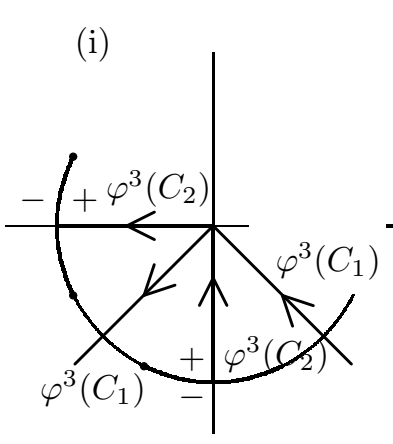

(ii)

(i)

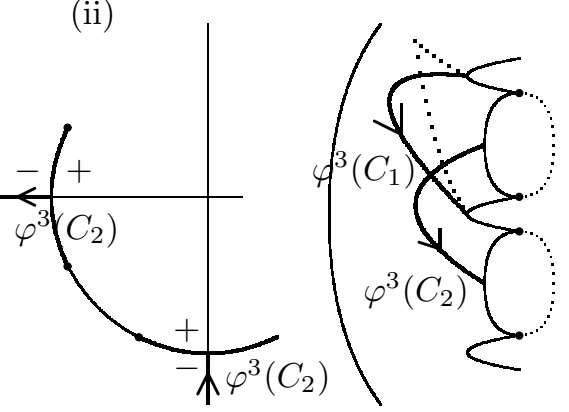

(i)

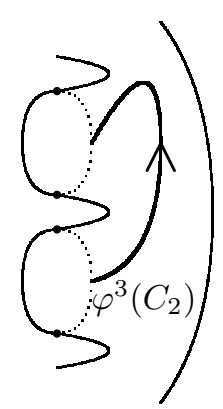

(ii)

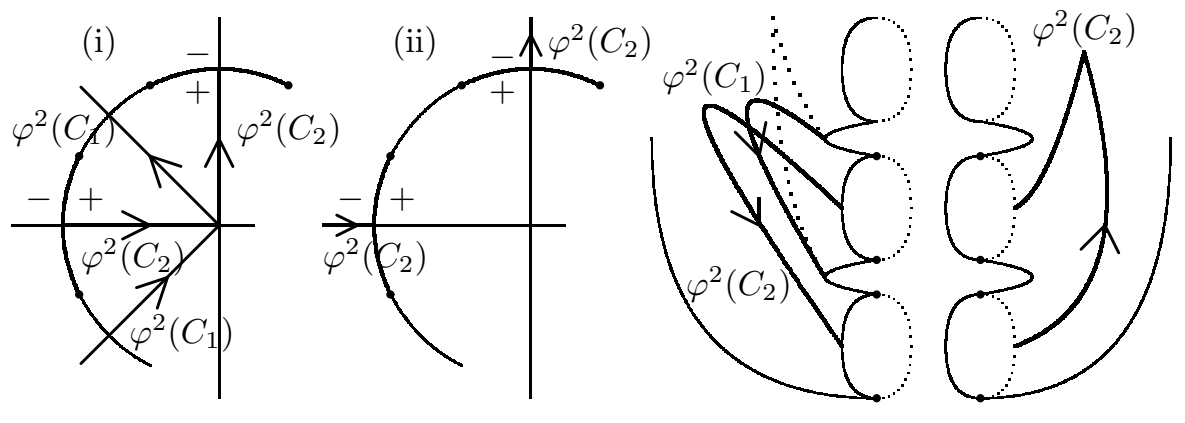


Therefore, we find a canonical homology basis as follows.

$$
\begin{aligned}
& A_{1}=C_{2}, \quad B_{1}=C_{1}, \quad A_{2}=\varphi^{3}\left(C_{2}\right), \quad B_{2}=B_{1}+\varphi^{3}\left(C_{1}\right), \\
& A_{3}=\varphi^{2}\left(C_{2}\right), \quad B_{3}=B_{2}+\varphi^{2}\left(C_{1}\right) .
\end{aligned}
$$

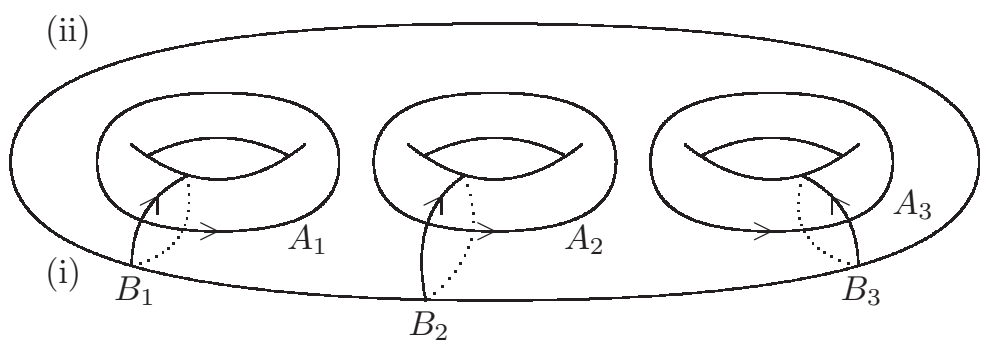

\subsection{2 period matrix}

Key 1-cycles of the canonical homology basis as in $\S 5.4 .1$ are given by $C_{1}$ and $C_{2}$. Straightforward calculation yields

$$
\begin{aligned}
\int_{C_{1}} \frac{1-z^{2}}{w} d z & =-\sqrt{2} i \int_{0}^{\infty} \frac{1-t^{2}}{\sqrt{t^{8}-a t^{4}+1}} d t=0 \\
\int_{C_{1}} \frac{i\left(1+z^{2}\right)}{w} d z & =2 \sqrt{2} \int_{0}^{1} \frac{1+t^{2}}{\sqrt{t^{8}-a t^{4}+1}} d t \\
\int_{C_{1}} \frac{2 z}{w} d z & =-8 i \int_{0}^{1} \frac{t}{{\sqrt{t^{8}-a t^{4}+1}}^{6}} d t \\
\int_{C_{1}} \frac{z^{4}-z^{6}}{w^{3}} d z & =\sqrt{2}^{2} \int_{0}^{\infty} \frac{t^{4}-t^{6}}{{\sqrt{t^{8}-a t^{4}+1}}^{3}} d t=0 \\
\int_{C_{1}} \frac{i\left(z^{4}+z^{6}\right)}{w^{3}} d z & =-2 \sqrt{2}_{0}^{1} \frac{t^{4}+t^{6}}{{\sqrt{t^{8}-a t^{4}+1}}^{3}} d t \\
\int_{C_{1}} \frac{z^{5}}{w^{3}} d z & =4 i \int_{0}^{1} \frac{t^{5}}{{\sqrt{t^{8}-a t^{4}+1}}^{3}} d t \\
\int_{C_{2}} \frac{i\left(1+z^{2}\right)}{w} d z & =-2 i \int_{0}^{\infty} \frac{1+t^{2}}{{\sqrt{t^{8}+a t^{4}+1}}^{1}} d t=-2 i \int_{0}^{1} \frac{1+t^{2}}{{\sqrt{t^{8}+a t^{4}+1}}^{2}} d t \\
\int_{C_{2}} \frac{2 z}{w} d z & =-8 \int_{0}^{1} \frac{t}{{\sqrt{t^{8}+a t^{4}+1}}^{2}} d t \\
\int_{C_{2}} \frac{z^{4}-z^{6}}{w^{3}} d z & =-i \int_{0}^{\infty} \frac{t^{4}+t^{6}}{{\sqrt{t^{8}+a t^{4}+1}}^{3}} d t=-2 i \int_{0}^{1} \frac{t^{4}+t^{6}}{{\sqrt{t^{8}+a t^{4}+1}}^{3}} d t
\end{aligned}
$$




$$
\begin{aligned}
\int_{C_{2}} \frac{i\left(z^{4}+z^{6}\right)}{w^{3}} d z & =-2 i \int_{0}^{1} \frac{t^{4}+t^{6}}{{\sqrt{t^{8}+a t^{4}+1}}^{3}} d t \\
\int_{C_{2}} \frac{z^{5}}{w^{3}} d z & =-4 \int_{0}^{1} \frac{t^{5}}{{\sqrt{t^{8}+a t^{4}+1}}^{3}} d t .
\end{aligned}
$$

By setting

$$
\begin{aligned}
& A=2 \sqrt{2} \int_{0}^{1} \frac{1+t^{2}}{\sqrt{t^{8}-a t^{4}+1}} d t, \quad B=2 \int_{0}^{1} \frac{1+t^{2}}{\sqrt{t^{8}+a t^{4}+1}} d t \\
& C=8 \int_{0}^{1} \frac{t}{{\sqrt{t^{8}+a t^{4}+1}}^{4}} d t, \quad D=8 \int_{0}^{1} \frac{t}{{\sqrt{t^{8}-a t^{4}+1}}^{6}} d t \\
& E=2 \sqrt{2}^{1} \frac{t^{4}+t^{6}}{{\sqrt{t^{8}-a t^{4}+1}}^{3}} d t, \quad F=2 \int_{0}^{1} \frac{t^{4}+t^{6}}{{\sqrt{t^{8}+a t^{4}+1}}^{3}} d t \\
& H=4 \int_{0}^{1} \frac{t^{5}}{{\sqrt{t^{8}+a t^{4}+1}}^{3}} d t, \quad I=4 \int_{0}^{1} \frac{t^{5}}{{\sqrt{t^{8}-a t^{4}+1}}^{3}} d t
\end{aligned}
$$

and (5.69)-(5.80), we have

$$
\int_{C_{1}} G=\left(\begin{array}{c}
0 \\
A \\
-i D \\
0 \\
-E \\
i I
\end{array}\right), \quad \int_{C_{2}} G=\left(\begin{array}{c}
-i B \\
-i B \\
-C \\
-i F \\
-i F \\
-H
\end{array}\right)
$$

Therefore, the period matrix of the abelian differentials of the second kind is given by

$$
\left(\begin{array}{cccccc}
-i B & i B & i B & 0 & -A & -A \\
-i B & -i B & i B & A & A & 0 \\
-C & C & -C & -i D & 0 & -i D \\
-i F & i F & i F & 0 & E & E \\
-i F & -i F & i F & -E & -E & 0 \\
-H & H & -H & i I & 0 & i I
\end{array}\right)
$$

\section{References}

[1] V. Cortés, On hyper-Kähler manifolds associated to Lagrangian Kähler submanifolds of $T^{*} \mathbb{C}^{n}$, Trans. Amer. Math. Soc. 350 (1998), no. 8, 3193-3205.

[2] N. Ejiri, A differential-geometric Schottky problem, and minimal surfaces in tori, Contemp. Math. 308 (2002), 101-144.

[3] N. Ejiri, A generating function of a complex Lagrangian cone in $\mathbb{H}^{n}$, preprint. 
[4] N. Ejiri and T. Shoda, On a moduli theory of minimal surfaces, Prospects of differential geometry and its related fields, (2014), 155-172, World Sci. Publ.

[5] N. Ejiri and T. Shoda, The existence of $r G$ family and $t G$ family, and their geometric invariants, preprint.

[6] P. Griffiths and J. Harris, Principles of Algebraic Geometry, Wiley Classics Library (1994).

[7] W. H. Meeks III, The theory of triply periodic minimal surfaces, Indiana Univ. Math. J. 39 (1990), no. 3, 877-936.

[8] S. Montiel and A. Ros, Schrödinger operators associated to a holomorphic map, Global differential geometry and global analysis, Lecture Notes in Math. 1481 (1991), 147-174.

[9] A. Ros, One-sided complete stable minimal surfaces, J. Differential Geom. 74 (2006), no. 1, 69-92.

[10] M. Ross, Schwarz' $P$ and D surfaces are stable, Differential Geom. Appl. 2 (1992), no. 2, 179-195.

[11] J. Sacks and K. Uhlenbeck, The existence of minimal immersions of 2spheres, Ann. of Math. (2) 113 (1981), no. 1, 1-24.

[12] R. Schoen and S. T. Yau, Existence of incompressible minimal surfaces and the topology of three-dimensional manifolds with nonnegative scalar curvature, Ann. of Math. (2) 110 (1979), no. 1, 127-142.

[13] J. Simons, Minimal varieties in riemannian manifolds, Ann. of Math. (2) 88 (1968), 62-105.

[14] G. E. Schröder-Turk, A. Fogden, and S. T. Hyde, Bicontinuous geometries and molecular self-assembly: comparison of local curvature and global packing variations in genus-three cubic, tetragonal and rhombohedral surfaces, Eur. Phys. J. B. 54 (2006), 509-524.

\author{
Norio Ejiri \\ Department of Mathematics, Meijo University \\ Tempaku, Nagoya 468-8502, Japan. \\ ejiri@meijo-u.ac.jp \\ Toshihiro Shoda \\ Faculty of Education, Saga University \\ 1 Honjo-machi, Saga-city, Saga, 840-8502, Japan. \\ tshoda@cc.saga-u.ac.jp
}

